University of Louisville

ThinkIR: The University of Louisville's Institutional Repository

Electronic Theses and Dissertations

8-2017

\title{
Circuits for active vision : parallel tectothalamocortical visual pathways in the mouse.
}

Na Zhou

University of Louisville

Follow this and additional works at: https://ir.library.louisville.edu/etd

Part of the Other Neuroscience and Neurobiology Commons

\section{Recommended Citation}

Zhou, $\mathrm{Na}$, "Circuits for active vision : parallel tectothalamocortical visual pathways in the mouse." (2017). Electronic Theses and Dissertations. Paper 2782.

https://doi.org/10.18297/etd/2782

This Doctoral Dissertation is brought to you for free and open access by ThinkIR: The University of Louisville's Institutional Repository. It has been accepted for inclusion in Electronic Theses and Dissertations by an authorized administrator of ThinkIR: The University of Louisville's Institutional Repository. This title appears here courtesy of the author, who has retained all other copyrights. For more information, please contact thinkir@louisville.edu. 
CIRCUITS FOR ACTIVE VISION: PARALLEL TECTOTHALAMOCORTICAL VISUAL PATHWAYS IN THE MOUSE

By

\author{
$\mathrm{Na}$ Zhou \\ B.S., Lanzhou University, China, 2010 \\ M.S., University of Louisville, 2014
}

\begin{abstract}
A Dissertation
Submitted to the Faculty of the

School of Medicine of the University of Louisville

In Partial Fulfillment of the Requirements

For the Degree of
\end{abstract}

Doctor of Philosophy in Anatomical Sciences and Neurobiology

Department of Anatomical Sciences and Neurobiology,

University of Louisville, School of Medicine

Louisville, Kentucky

August 2017 

CIRCUITS FOR ACTIVE VISION: PARALLEL TECTOTHALAMOCORTICAL VISUAL PATHWAYS IN THE MOUSE

By

\author{
Na Zhou \\ B.S., Lanzhou University, China, 2010 \\ M.S., University of Louisville, 2014
}

A Dissertation Approved on

June 23, 2017

By the following Dissertation Committee:

Martha E. Bickford, PhD.

Dissertation Director

William Guido, PhD.

Heywood M. Petry, PhD.

Robert F. Lundy, PhD.

David SK. Magnuson, PhD.

Jeffrey C. Petruska, PhD. 
To my parents

Mrs. Jianhua Wang and Mr. Fengnian Zhou for their love and support to me 


\section{ACKNOWLEDGMENTS}

I would like to express my deepest gratitude to my mentor, Dr. Martha E. Bickford, for her continuous guidance, encouragement, caring and patience over the past six years. She has set an excellent role model for me to pursue my science career and to achieve my life goals. She has inspired my passion for science. I couldn't forget those moments when she was hanging around expecting to see what would happen in my experiments and I also cherished those times when we tried all sorts of different experiments no matter whether it is a failure or not and no matter how late it gets. It is her never-give-up spirit that keeps me going and guide me through all obstacles on this road. I have enjoyed my past six years in her lab, and I have learned a lot. I thank her again here and hope she would like this dissertation.

I would also like to thank my other committee members Dr. William Guido, Dr. Heywood M. Petry, Robert F. Lundy, David SK. Magnuson and Jeffrey C. Petruska for their guidance and help. I thank Dr. Guido for supporting me financially for a year and advice which pushed me digging deeper under the big picture. I am really grateful for his sharing equipment with us, especially for me to be able to use confocal microscopy and zoom scope. I thank Dr. Petry for mentored me for a semester on in vivo tree shrew studies that were carried in his laboratory; it was the 
first time in my life that I have met the magic creature-tree shrew. I thank Dr. Lundy for me rotating in his lab to learn how to conduct in vivo taste experiments in rats and for his kindness to let me use his montage epifluorescence microscope. I thank Dr. Magnuson for his suggestions on my experiments, and his good habit to make notes on individual students that he advises impressed me a lot. I thank Dr. Petruska for his suggestions and also his support for Sean and me learning his lab's electrophysiology tricks from Kris. I thank Dr. Nigel G. Cooper, for him letting me rotating in his lab in my first semester and learned real time PCR technique and also his guidance on how to write research project report.

Additionally, I would also like to thank my previous and current fellow lab mates, Dr. Haiyang Wei, Arkadiusz S. Slusarczyk and Dr. Sean P. Masterson for their knowledge and guidance for numerous lab techniques and tricks. I am very thankful for Sean's contribution to all the maintenance and all the cells he recorded for my project. Thanks for Phillip S. Maire helping me manage the mice colony. Finally, I would like to thank all previous rotation lab members Xiangping Wang, Xiaohong Li and Yi Kang for teaching me their skills. I'm very grateful for the opportunity to be in such a wonderful research environment.

Last but not least, I would like to thank my family and friends for mental supporting. Especially for my friends Judy C. Hollkamp and Yi Kang, I thank them for being there all the time for me, always caring me and guiding me through my frustrating times. 


\begin{abstract}
CIRCUITS FOR ACTIVE VISION: PARALLEL TECTOTHALAMOCORTICAL VISUAL PATHWAYS IN THE MOUSE
\end{abstract}

$\mathrm{Na}$ Zhou

June 23, 2017

Vision is a critical sensation for the interaction between humans and their surrounding environment. The eyes connect with the brain via retinal ganglion cell axons, which transmit visual sensory information from the periphery into the central nervous system for further processing, eventually leading to visual perception and the visual guidance of movement. Two main targets of retinal axons are the superior colliculus (SC) and the dorsal thalamus. From the SC, visual information is conveyed to the dorsal thalamus, and from the dorsal thalamus visual information is conveyed to the cortex, striatum and amygdala. This dissertation is focused on the functional properties of two parallel pathways from the SC to the dorsal thalamus: a pathway from the $\mathrm{SC}$ to the retinorecipient dorsolateral geniculate nucleus (dLGN) to the cortex, and a pathway from the SC to the pulvinar nucleus to the cortex.

The experiments described in this dissertation used viral vector injections, tract tracing, in vitro whole cell patch clamp, optogenetics, electron and confocal microscopy, transgenic mouse lines and immunohistochemical staining techniques to 
elucidate the roles of the SC-dLGN-cortex pathway and SC-pulvinar-cortex pathway in visual coding. The first series of experiments revealed that SC and retinal inputs converge to innervate the proximal dendrites of cells in the dorsolateral shell of the dLGN that project to layer I of the striate cortex. The second series of experiments revealed the organization of subdivisions of the pulvinar nucleus in relation to inputs from the SC. The final series of experiments revealed the distribution and ultrastructure of pulvinocortical terminals, and identified the cell types activated by pulvinocortical synapses. Major targets of pulvinocortical terminals were identified as corticostriatal cells, suggesting that pulvinar acts as a hub connecting the SC, cortex and striatum. 


\section{TABLE OF CONTENTS}

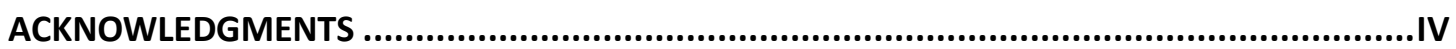

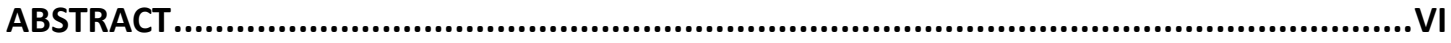

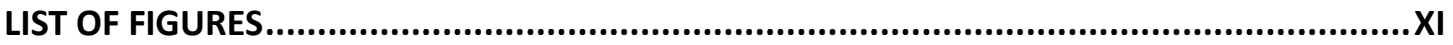

\section{CHAPTER}

I. INTRODUCTION

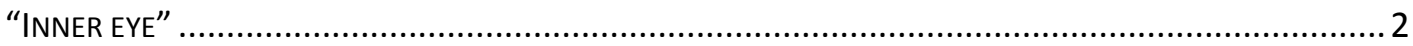

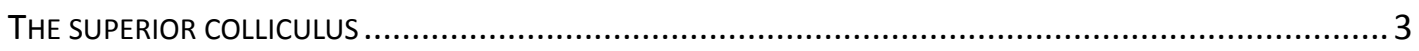

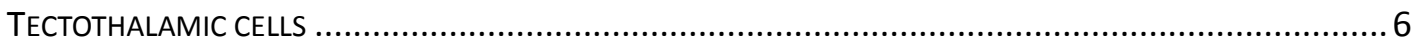

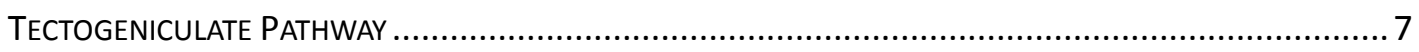

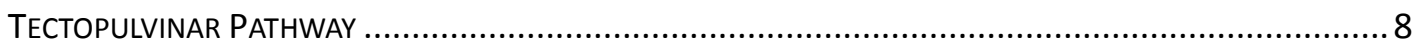

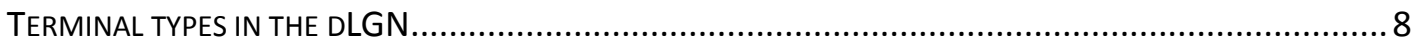

ULTRASTRUCTURE OF "DRIVERS" AND “MODULATORS” IN THE DLGN ........................................... 10

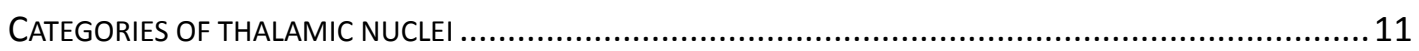

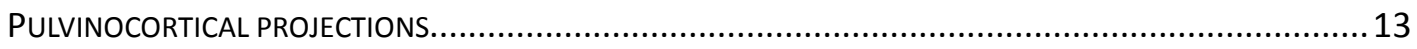

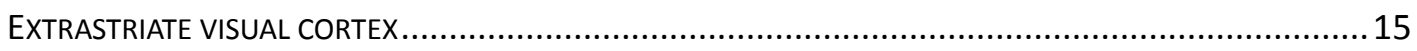

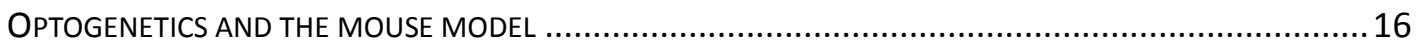

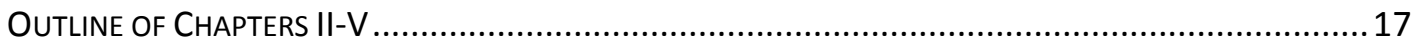

II. RETINAL AND TECTAL “DRIVER-LIKE” INPUTS CONVERGE IN THE SHELL OF THE MOUSE DORSAL LATERAL GENICULATE NUCLEUS ........................................... 18

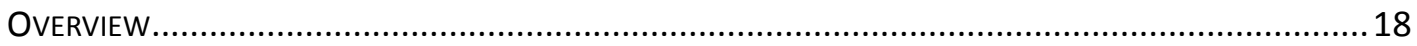

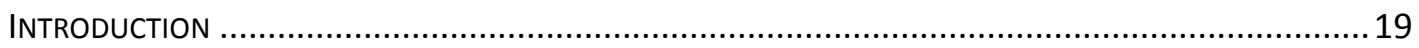

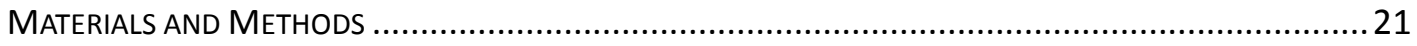

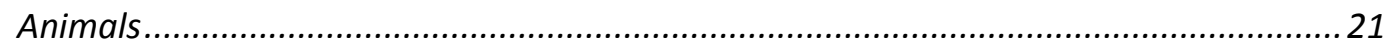

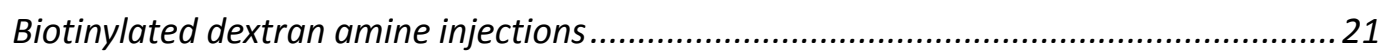

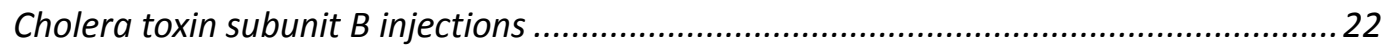

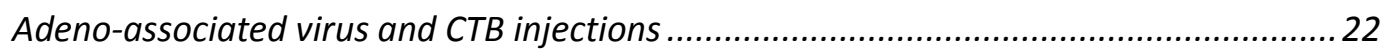

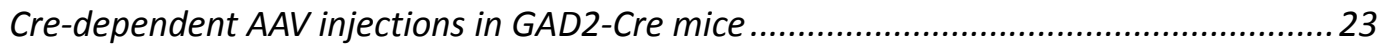

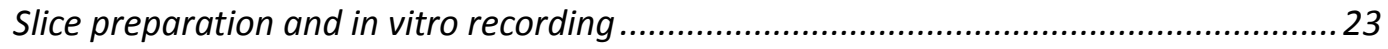

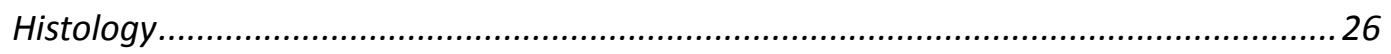

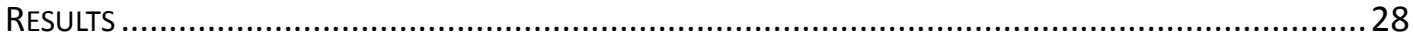

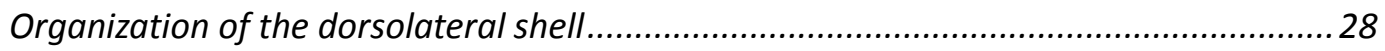

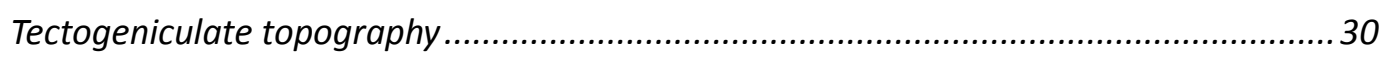

The vast majority of tectogeniculate projections are non-GABAergic ............................ 30 
TG terminals: a new class of dLGN synaptic profile

TG responses are "driver-like" and converge with $R G$ inputs ........................................ 34

Neurons postsynaptic to TG terminals contact non-GABAergic dendrites in V1 layer I ... 36

DISCUSSION

\section{THE MOUSE PULVINAR NUCLEUS: ORGANIZATION OF THE TECTORECIPIENT ZONES}

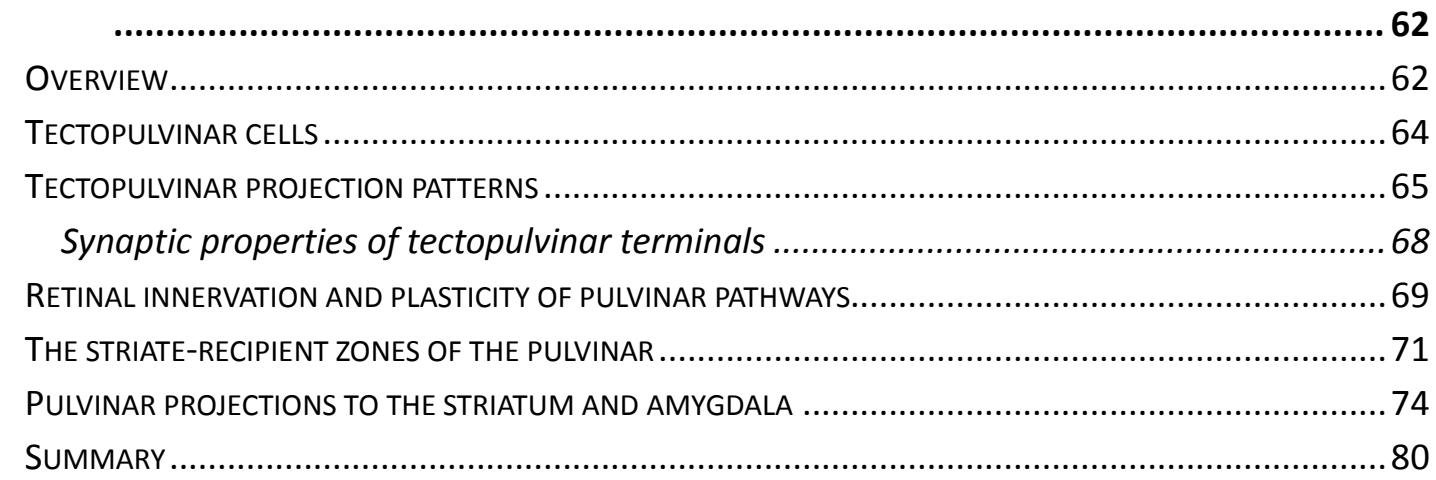

IV. THE MOUSE PULVINAR NUCLEUS LINKS THE LATERAL EXTRASTRIATE CORTEX, STRIATUM, AND AMYGDALA: CELL TYPE-SPECIFIC THALAMOCORTICAL CIRCUIT

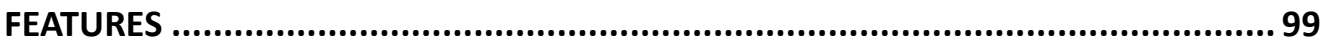

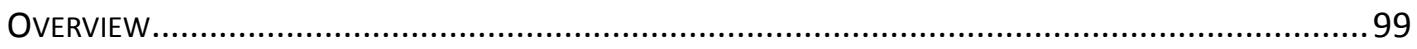

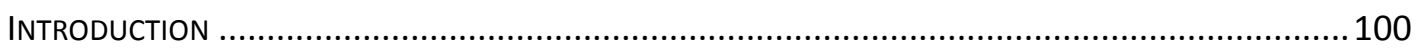

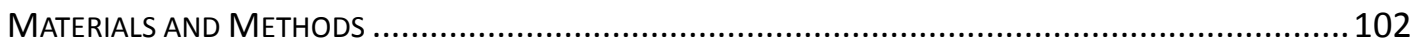

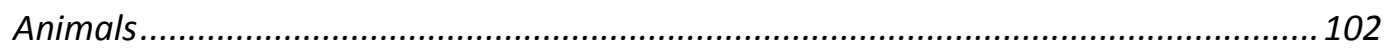

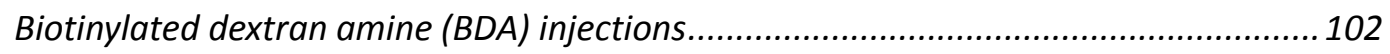

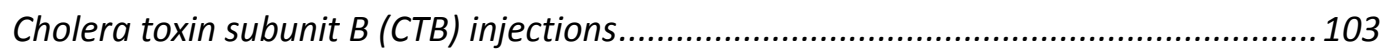

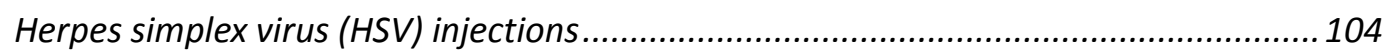

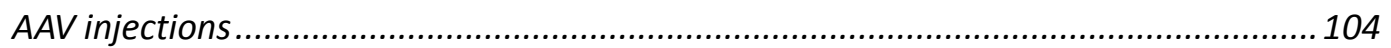

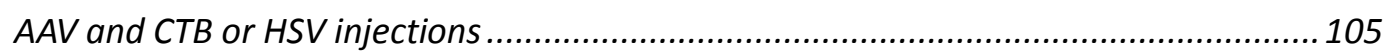

Slice preparation and optogenetic stimulation ......................................................... 105

Processing of cells filled during physiological recording .............................................. 108

Histology for tissue used for anatomical analyses .....................................................109

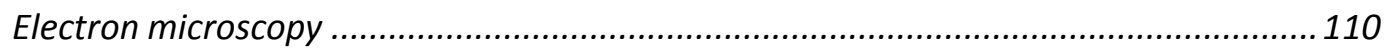

Experimental design and statistical analyses ..........................................................111

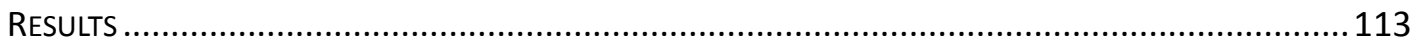

Extrinsic projections of the mouse pulvinar: comparison with the dLGN..................... 113

Optogenetic activation of thalamocortical terminals that originate from the pulvinar 115

Morphology and location of recorded cortical neurons ............................................. 116

Cell types responsive to optogenetic activation of pulvinocortical terminals ................117

Optogenetic activation of pulvinar terminals: effects on cortical interneurons..............118

Optogenetic activation of pulvinar terminals: effects on cortical output neurons ........120

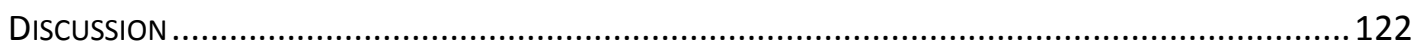

Morphology of thalamocortical terminals and their postsynaptic targets ....................122

Projection neurons of the mouse visual cortex............................................................. 124

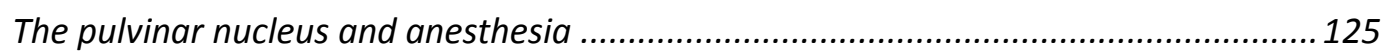

Is the pulvinar nucleus a hub for visually-triggered action selection? ...........................126

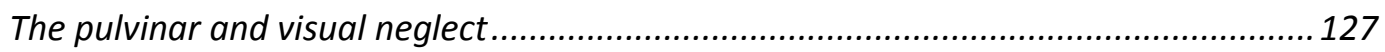




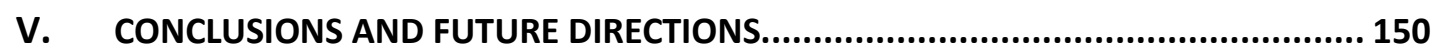

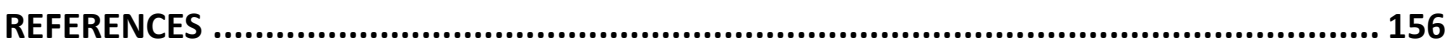

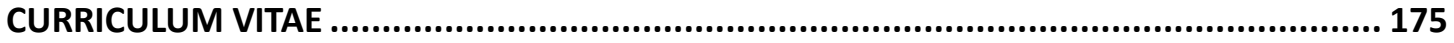




\section{LIST OF FIGURES}

FIGURE

Figure 1. Components of the dLGN dorsolateral shell........................................42

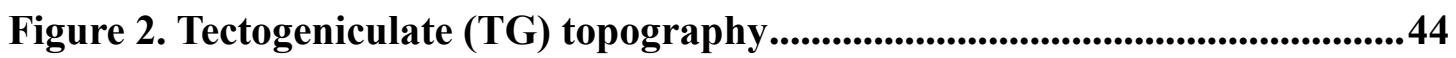

Figure 3. Tectogeniculate projections are primarily nonGABAergic ....................46

Figure 4. Ultrastructure of tectogeniculate (TG) terminals ..................................48

Figure 5. Light-evoked tectogeniculate (TG) responses ......................................50

Figure 6. Synaptic depression of tectogeniculate (TG) responses..........................52

Figure 7. Functional convergence of TG and RG inputs........................................54

Figure 8. Tectorecipient dLGN neurons project to V1 layer I .............................56

Figure 9. Synaptic targets of dLGN projections to V1 layer I ..............................58

Figure 10. Distinct functional circuits in the dLGN core and shell .......................60

Figure 11. The pulvinar nucleus contains two tectorecipient zones ........................81

Figure 12. Widefield vertical (WFV) cells project to the ipsilateral and contralateral pulvinar .83 
Figure 13. Caudal medial pulvinar (Pcm) cells express calretinin (CR) and neurokinin 1 (NK1) and align with bilateral superior colliculus (SC) projections .85

Figure 14. The Pcm contains a dense population of terminals that contain substance $\mathbf{P}$ .87

Figure 15. Ultrastructure of cortical and tectal terminals in the mouse pulvinar

Figure 16. Tectopulvinar and corticopulvinar terminals overlap in the caudal medial (Pcm) and lateral (PI) subdivisions of the mouse pulvinar .91

Figure 17. Potential input integration in the mouse pulvinar .93

Figure 18. The mouse pulvinar projects to the cortex, striatum and amygdala ..95

Figure 19. The tectorecipient mouse pulvinar forms interconnected loops with the cortex, striatum and amygdala

Figure 20. Laminar distribution and ultrastructure of thalamocortical terminals originating from the dLGN and pulvinar 128

Figure 21. Optogenetic activation of thalamocortical terminals that originate from the pulvinar 130

Figure 22. Pulvinar activation of pyramidal cells 132

Figure 23. Pulvinar activation of spiny stellate cells

Figure 24. Creation of flattened map from coronal sections 136

Figure 25. Distribution of cells that activated by the pulvinar 138

Figure 26. Characterization of GAD65-GFP cells in V1 140 
Figure 28. Interneuron properties 144

Figure 29. Pulvinar activation of extrinsic projection cells ..................................146

Figure 30. Summary of pulvinocortical circuits.............................................................. 148

Figure 31. Future DREADDS experiment .......................................................154 


\section{CHAPTER 1}

\section{INTRODUCTION}

Vision is not a simple representation of the surrounding environment; it also plays a crucial role in guiding an organism's interaction with the environment. Thus, vision includes two components, visual perception and visuomotor guidance. The classic primary visual pathway is from the retina to layer IV of the primary visual cortex (or striate cortex), via the dorsolateral geniculate nucleus (dLGN) of the dorsal thalamus (Diamond and Hall, 1969; LeVay and Gilbert, 1976; Peters and Feldman, 1977). This pathway is critical for visual perception because patients do not perceive their environment following lesions of the striate cortex (Weiskrantz, 1986). The retina also sends axons to innervate many other subcortical nuclei, including the superficial layers of the superior colliculus (SC or optic tectum) in the midbrain. From the superficial layers of the SC, there are two parallel pathways to the dorsal thalamus. One tectothalamic pathway transmits visual information to the pulvinar nucleus, which is subsequently forwarded to the striate and extrastriate cortex, as well as the striatum and amygdala. The other tectothalamic pathway innervates subregions of the dLGN to form a component of the geniculostriate pathway (Diamond and Hall, 1969; Schneider, 1969; Casagrande et al., 1972). These retino-tecto-thalamocortical pathways are known as secondary visual pathways, 
which are considered to play important roles in visual transmission that are independent of that served by the primary retino-geniculo-cortical pathway.

However, the function of the secondary visual pathways is not fully understood. This dissertation focuses on the tecto-geniculo-cortical and tecto-pulvinocortical pathways, using the mouse as a model, with the goal of understanding the underlying synaptic features of these pathways. This information is a necessary first step toward a complete understanding of the functions served by these two parallel tectothalamic pathways.

\section{"Inner eye"}

Weiskrantz and his colleagues coined the term "blindsight" to describe the phenomenon that, in spite being unaware of visual stimuli, residual visual abilities are preserved in patients with damage to the visual cortex, and this residual vision can guide movements (Weiskrantz et al., 1974). Blindsight patients could discriminate simple properties such as small spots of light in their blind visual receptive field (reported via eye movements or pointing). They could also distinguish straight from curved lines, stimuli differing in color, or the orientation and spatial frequency of visual stimulus gratings (Weiskrantz, 1986). Furthermore, blindsight patients could also detect the emotional expression of faces (de Gelder et al., 1999; Van den Stock et al., 2011), rapidly moving stimuli, rapid changes in brightness, the onset of motion, or the direction and velocity of isolated moving targets (Weiskrantz, 1986). However, blindsight patients fail to identify the direction of motion of patterns composed of multiple components (Azzopardi and Cowey, 2001). 
In addition to the visual capabilities demonstrated in human patients with striate cortex damage, experimental evidence indicates that in some species complete removal of the striate cortex (resulting in complete degeneration of the dLGN), does not impair discrimination of simple patterns, colors, or acuity (Snyder et al., 1966; Snyder and Diamond, 1968; Ware et al., 1972, 1974). These spared visual abilities are likely to rely on the secondary visual pathway from the SC to the pulvinar nucleus (Stoerig and Cowey, 1997). Thus, consciousness and unconsciousness visual functions may be served by the primary and secondary visual pathways respectively. However, the functions of tectothalamocortical pathways are still unclear. This dissertation examines the synaptic organization of these pathways in the mouse as a first step toward determining their function.

\section{The superior colliculus}

The SC is a highly-laminated area in all mammals. Regions of the SC that are primarily visually-responsive are the superficial layers, which include (from dorsal to ventral) the stratum zonale (SZ), the stratum griseum superficiale (SGS), and the stratum opticum (SO) (Dräger and Hubel, 1975). Retinotectal terminals are mainly located in the SZ and SGS; few are located in the SO (Schönitzer and Holländer, 1984). The SC receives bifurcating projections from single retinal ganglion cells (RGC) that branch to the dLGN (Kondo et al., 1993, 1994; Dong et al., 1995; Ellis et al., 2016). It has been reported that almost all RGC types innervate the $\mathrm{SC}$ in rabbits and over $90 \%$ of RGC neurons directly project to the SC in rats (Vaney et al., 1981; Linden and Perry, 1983; Dean and Redgrave, 1984). In mice, functional 
characterization of RGCs revealed that there are several classes of SC-projecting RGCs that avoid the dLGN, and these RGCs appear to exhibit more transient responses and respond best to small stimuli (Ellis et al., 2016).

Retinal inputs first invade the SC as early as embryonic day 15 (E15) in the mouse (Edwards et al., 1986), and activity-dependent mechanisms establish a precise topography of retinotectal inputs (Chandrasekaran et al., 2005). The nasal retina of the contralateral eye and the temporal retina of both eyes innervate the SC (Rapaport and Wilson, 1983). The nasotemporal and ventrodorsal axes of the retina projects onto the caudorostral and mediolateral axes of the contralateral SC respectively (Sauvé et al., 2001). The retinotopic organization of the $\mathrm{SC}$ has been reported to be consistent among different mammals, with the zero vertical meridian represented rostrally, and the most peripheral part of the contralateral visual field represented caudally; the upper visual field is represented medially/rostrally in the SC, and the lower visual field is represented laterally/caudally in the SC (Berman and Cynader, 1972; Cynader and Berman, 1972; Dräger and Hubel, 1975, 1976; Bickford et al., 2015).

In vivo recording studies have shown that superficial SC cells exhibit antagonistic center-surround receptive fields, in which excitatory responses are evoked when stimuli are presented in the center region of the receptive field, whereas visual stimuli can cause surrounding suppression if they presented in the surround area. It has been suggested that secondary visual pathways, in comparison to the geniculostriate pathway, may provide a stronger representation of the peripheral visual field and are more sensitive to small transient visual stimuli (Dean and Redgrave, 1984). 
In contrast to the superficial SC, the deep layers of SC are comprised of multisensory neurons and premotor neurons, which receive inputs from various nonvisual sensory structures and send descending/ascending projections to the brainstem, spinal cord, and thalamus. Ample evidence suggests that the deep layers of $\mathrm{SC}$ are involved in the translation of sensory signals into motor commands in order to orient eye, head, hand, trunk and pinnae movements (Sparks and Hartwich-Young, 1989; Isa, 2002). Two studies in hamster found that following damage restricted to the superficial layers of SC, animals experienced a near loss of orienting to sunflower seeds in the upper and peripheral visual field, whereas if the damage also destroyed or undercut the deep layers of SC, the hamsters' ability to orient was completely abolished (Finlay et al., 1980; Mort et al., 1980). Similarly, an early tree shrew study suggested that the superficial layers and deep layers of SC channel different functions, and the behavioral effects caused by lesions in the deep layers partially depended on the input from the superficial layers (Casagrande et al., 1972). However, how information is transmitted between the superficial layers and deep layers of SC is still largely unknown.

The importance of the balance of activity across visual areas for proper visuomotor control was demonstrated by the phenomenon known as the "Sprague effect" (Sprague, 1966). Sprague found that in cats, unilateral visual cortex damage caused a "visual neglect" of one hemifield (detection, attention or orientation visual deficits in the lesioned visual hemifield), but subsequent lesions of the contralateral 
SC relieved this neglect, and animals regained their ability to orient toward the previously neglected hemifield (Sprague, 1966).

\section{Tectothalamic cells}

A ubiquitous laminar segregation of SC neurons that project to the dLGN or the pulvinar (or lateral posterior nucleus, LPN) has been described in a number of species including cat (Straschill and Hoffmann, 1969; Kawamura et al., 1980), tree shrew (Albano et al., 1979; Graham and Casagrande, 1980), hamster (Rhoades and Chalupa, 1976; Mooney et al., 1988), rat (Donnelly et al., 1983), rabbit (Graham and Berman, 1981), squirrel monkey (Kadoya et al., 1971) and grey squirrel (Robson and Hall, 1977). Neurons that innervate the dLGN are always located in the upper onehalf of the stratum griseum superficiale (SGS) and the majority of these cells are narrow field vertical cells (NFV), whereas those neurons that project to the pulvinar are located in the lower half of the SGS and stratum opticum (SO), and the majority of these cells are wide field vertical cells (WFV) (Mooney et al., 1988; Chomsung et al., 2008; Fredes et al., 2012; Gale and Murphy, 2014). It has also been noted that the somata of tectopulvinar cells are larger than those of tectogeniculate neurons (Albano et al., 1979; Kawamura et al., 1980; Caldwell and Mize, 1981; Graham and Berman, 1981; Harrell et al., 1982). NFV cells have narrow dendritic fields that extend dorsally and ventrally from their somata; these dendrites are oriented perpendicular to the dorsal surface of the SC. WFV or tectopulvinar cells have very wide dendritic fields that extend dorsally from their somata. The distal portions of these dendrites end either in the most superficial SGS, or in the middle of the SGS, and their tips exhibit 
unique bottlebrush endings (arrays of spiny dendritic tufts).( Karten et al., 1997; Luksch et al., 1998; Major et al., 2000). WFV cells have large receptive fields and respond to small stimuli that move in any direction. In contrast, NFV cells may be specialized to code more detailed motion parameters based on their small receptive fields and strong direction selectivity (Gale and Murphy, 2014).

\section{Tectogeniculate Pathway}

SC input to the dLGN has been observed in 19 different mammalian species (Harting et al., 1991). In the primate, tectal inputs project to the koniocellular layers of the dLGN; these layers subsequently project to layer 1 of the striate cortex (Diamond et al., 1991; Harting et al., 1991). Likewise, in the tree shrew, tectal input innervates layers 3 and 6 of the dLGN and these dLGN layers project to supragranular layers in V1. In rodents, tectogeniculate inputs innervate the dorsolateral shell, a region located along a thin strip of the dLGN that runs adjacent and parallel to the optic tract (Reese, 1984; Harting et al., 1991; Grubb and Thompson, 2004). Interestingly, at least 3 classes of identified direction-selective retinal ganglion cells project to the dorsolateral shell, as well as to the SGS (Kim et al., 2008, 2010; Huberman et al., 2009; Kay et al., 2011; Rivlin-Etzion et al., 2011). A recent study of mouse NFV cells reported that all were direction selective (Gale and Murphy, 2014). Moreover, studies of the receptive field properties of mouse dLGN neurons have revealed that direction-selective cells are specifically located in the dorsolateral shell (Marshel et al., 2012; Piscopo et al., 2013). These studies suggest a possible convergence of projections from direction-selective NFV cells and direction-selective 
ganglion cells within regions of the mouse dLGN that exhibit direction-selective responses. Chapter II of this dissertation will describe the underlying circuitry of this pathway as a first step toward understanding its function and roles in the visual system.

\section{Tectopulvinar Pathway}

In 1949, Rose and Woolsey proposed that the pulvinar and LPN are intrinsic thalamic nuclei, receiving information relayed by the dLGN (Rose and Woolsey, 1949). However, Karten and Revzin described a projection from the tectum to the nucleus rotundus in birds via tract tracing techniques in 1966 (Karten and Revzin, 1966), and subsequently in 1973 Harting confirmed that the pulvinar is heavily innervated by the SC instead of the dLGN using lesion techniques in tree shrews (Harting et al., 1973a, 1973b). Since these initial studies, various features of tectopulvinar pathways have been studied and these are reviewed in Chapter III of this dissertation.

\section{Terminal types in the dLGN}

In 1969, four basic terminal types were identified in the cat dLGN which were designated RLP, RS, F1 and F2 profiles (Guillery, 1969). RLP profiles (large profiles with round vesicles and pale mitochondria) are glutamatergic terminals that have been found to originate from the retina (Rafols and Valverde, 1973; Robson and Mason, 1979; Rapisardi and Miles, 1984; Wilson et al., 1984). These large terminals contain mitochondria that have widened cristae, making them appear paler than surrounding mitochondria. RLP profiles form asymmetrical synapses, with more thickening of the 
postsynaptic density relative to the presynaptic density. In the mammalian brain, most excitatory synapses are asymmetrical with round vesicles whereas most inhibitory synapses are symmetrical with flattened or pleomorphic vesicles. RS profiles (small profiles with round vesicles) are either glutamatergic terminals that originate from the cortex or cholinergic terminals that originate from the parabigeminal nucleus (PBG) or the pedunculopontine tegmentum (PPT), although some are noradrenergic or serotonergic terminals (Jones and Powell, 1969; Erişir et al., 1998). F1 profiles (originally named for their vesicles which flatten with certain fixation procedures) contain gamma aminobutyric acid (GABA) and densely packed vesicles, and form symmetric synapses. These terminals originate from the thalamic reticular nucleus (TRN), pretectum (PT) and dLGN interneuron axons and are strictly presynaptic (Ohara et al., 1980; Montero and Scott, 1981; Fitzpatrick et al., 1984; Wilson et al., 1984; Hamos et al., 1985; Montero and Singer, 1985; Arcelli et al., 1997; Wang et al., 2001, 2002; Li et al., 2003c). F2 profiles are GABAergic terminals that originate from the dendrites of dLGN interneurons and are both presynaptic and postsynaptic (Bickford et al., 2000, 2010). The vesicles in F2 profiles are more sparsely distributed than those contained within F1 profiles.

In cat dLGN, axonal F1 terminals form simple, conventional synaptic contact onto the dendrites of thalamocortical cells; whereas the F2 dendritic terminals are both postsynaptic to various terminals and presynaptic to thalamocortical cells (Datskovskaia et al., 2001a; Dankowski and Bickford, 2003). There are many more F2 outputs per interneuron than F1 outputs (Sherman, 2004). 
RLPs are associated with a remarkable synaptic arrangement called a glomerulus, which contains RLP terminals, F terminals (both F1 and F2), and RS terminals from the brainstem. Within these glomeruli, the RLP profiles often form triads in which the RLP profile contacts both a relay cell dendrite and an F2 profile, and this same F2 profile is also presynaptic to the same relay cell dendritic appendage or shaft that is contacted by the RLP profile. Subsequent investigations of the dLGN identified a $5^{\text {th }}$ terminal type, designated RLD (round vesicles, large profile with dark mitochondria), which originates either from the axon collaterals of geniculocortical cells (Bickford et al., 2008) or the PPT (Erisir et al., 1997).

\section{Ultrastructure of "drivers" and "modulators" in the dLGN}

Even though the vast majority of synapses in the dLGN originate from nonretinal inputs (Guillery, 1969; Wilson et al., 1984; Van Horn et al., 2000), the receptive field properties of dLGN relay cells have been found to be nearly identical to those of their presynaptic retinal inputs (Cleland et al., 1971). Thus, retinal inputs have been defined as the "drivers" of geniculate activity (Sherman and Guillery, 1998), and inputs that exhibit similar features (i.e. large glutamatergic inputs that synapse on proximal dendrites) have also been classified as driving inputs (Sherman and Guillery, 1998). In contrast, corticogeniculate inputs have been found to have more subtle effects on dLGN neurons (Kalil and Behan, 1987; Sillito and Jones, 2002), so these inputs have been classified as "modulators" of geniculate activity and inputs that exhibit similar features (smaller terminals that innervate more distal dendrites) are considered to exert more subtle effects on thalamic activity. Although 
ultrastructure alone cannot determine the effect of input on its postsynaptic target, it provides a starting point for understanding its influence relative to other inputs. As described in Chapter II, the ultrastructure of tectogeniculate inputs was found to be correlated with their influence on geniculate neurons relative to retinal inputs.

\section{Categories of thalamic nuclei}

As proposed by Sherman and Guillery (1998), the thalamus contains two basic types of nuclei: first order nuclei and higher order nuclei, which are categorized based on the source of their "driver" inputs. First order nuclei receive their driving inputs from peripheral noncortical sources, whereas higher order nuclei receive their driving afferents from layer $\mathrm{V}$ cortical cells within the visual, auditory and somatosensory systems (Shepherd, 2004). At least some subdivisions of the pulvinar nucleus can be considered "higher order" because they receive input from layer V cells of the visual cortex (Purushothaman et al. 2012).

Anatomical studies have shown that layer V neurons in the striate cortex form relatively large terminals in the pulvinar, whereas neurons in layer VI of striate cortex form smaller terminals in the pulvinar nucleus (Trojanowski and Jacobson, 1977; Mason and Groos, 1981; Abramson and Chalupa, 1985; Bourassa and Deschênes, 1995; Vidnyánszky et al., 1996; Feig and Harting, 1998). In general, the tectorecipient pulvinar subdivisions receive afferent projections that originate exclusively from layer VI of the extrastriate cortex (Kawamura and Diamond, 1978; Chomsung et al., 2010). These corticopulvinar synapses from extrastriate cortex have been found to be located distal to the tectopulvinar synapses on the dendritic arbors of pulvinocortical cells, 
suggesting that the SC provides the "driving" input to the pulvinar neurons

(Chomsung et al., 2010). However, as reviewed in Chapter III, Masterson et al. (2009, 2010) proposed that tectorecipient subdivisions of the pulvinar nucleus should be considered as "second order" rather than "first order" or "higher order", because tectal terminals do not exhibit the characteristic of either the "drivers" or "modulators" defined by Sherman and Guillery 1998. In addition, as described in Chapter II, certain regions of the dLGN also do not fit the criteria or first or higher order nuclei.

\section{Functional properties of the pulvinar nucleus}

The pulvinar nucleus is the largest thalamic nucleus in primates, accounting for a quarter of the total thalamic mass (Grieve et al., 2000). The pulvinar nucleus is composed of several subdivisions based on its inputs and outputs (details discussed in Chapter III) and therefore multiple pulvinar functions have been described such as higher-order visual and visuomotor transduction, organization of saccades (Robinson et al., 1986), visual attention (Van Essen, 2005) and visual salience (inhibition of inappropriate eye movements) (Robinson and Petersen, 1992). It has been shown that the pulvinar integrates object feature and spatial properties (Benevento and Port, 1995) and damage of the pulvinar may elicit contralateral visual neglect (Wilke et al., 2010).

The receptive field properties of neurons in pulvinar resemble those in the SC to a certain degree. Similar to neurons in SC, single neuron recording studies from awake monkey have shown that cells in inferior and lateral pulvinar (PI and PL) respond to stimulus movement over a wide range of speeds. PI neurons respond to 
spots of light and inhibitory surrounds, possess relatively large receptive fields that increased with eccentricity and have minimal presaccadic activity (Robinson et al., 1991; Berman and Wurtz, 2011). However, there are differences between the receptive fields of SC and pulvinar neurons. In cats, tecto recipient pulvinar neurons have large receptive fields which are twice the size of the average receptive fields recorded in the superficial SC. Most superficial SC cells in cats prefer a horizontal movement of visual targets, but pulvinar neurons do not have this preference. Some $\mathrm{SC}$ recipient pulvinar neurons show orientation selectivity, but this feature has never been seen in superficial SC (Mason, 1981; Chalupa and Abramson, 1988;

Rauschecker, 1988). Responses of tectorecipient pulvinar cells are facilitated by binocular stimulation, but SC cells are not (Chalupa and Abramson, 1988).

Studies of different primate species and humans have shown that there are two retinotopic maps within the inferior pulvinar (Gattass et al., 1978; Standage and Benevento, 1983; Li et al., 2013; Arcaro et al., 2015; Allen et al., 2016). In humans and macaque monkeys, these maps represent the contralateral visual field, with the lower visual field represented dorsally and the upper visual field represented ventrally. In macaque monkeys, there is an expanded representation of central vision located caudally within the crescent of the pulvinar, while peripheral vision is represented rostrally (Standage and Benevento, 1983).

\section{Pulvinocortical projections}

In 1949, Rose and Woolsey made significant progress in understanding the connections of the pulvinar nucleus using the Nauta degeneration method. With small 
lesions in the extrastriate cortex, no apparent degeneration was found either in the dLGN or pulvinar. Surprisingly, with more extensive large lesions in the same region, they found only the pulvinar nucleus showed degeneration (Rose and Woolsey, 1949). Thus, extensive areas of the cortex in primates are connected to the pulvinar nucleus. It has been suggested that the higher order regions of the pulvinar play a potential role in transthalamic corticocortical communication (Sherman and Guillery, 2011), while the second order tectorecipient pulvinar is involved in coordinating the appropriate motor reactions in response to visual signals (Day-Brown et al., 2010). In primates, the pulvinar neurons that project to that striate cortex are distinct from neurons that project to the extrastriate cortex, based on their different arbor sizes and terminal fields; this suggests that pulvinar projections to different cortical areas may have different functions (Florence and Casagrande, 1987). Chomsung et al (2010) provided first and the only morphological description of the synaptic connections of pulvinocortical terminals published to date; they found that pulvinocortical terminals originating from the tectorecipient zones of the pulvinar nucleus densely innervate layers of I-IV of the extrastriate cortex and synapse with spines that didn't contain gamma amino butyric acid (GABA). It has been found that activation or inactivation of the pulvinar nucleus can increase or decrease overall cortical activity levels and alter the directional and/or orientation selectivity of cortical neurons (Soares et al., 2004; Saalmann and Kastner, 2011; Purushothaman et al., 2012). However, it is currently unknown how the tectorecipient pulvinar affects cortical receptive field 
properties. Its extensive input from superior colliculus may critically shape the functional organization of its output to the visual cortex.

\section{Extrastriate visual cortex}

Studies have suggested that there are at least 9 higher visual areas that exist in mouse and each area includes a distinct retinotopic representation and displays a unique combination of spatiotemporal features (Wang and Burkhalter, 2007; Marshel et al., 2011). These areas are known as posterior, postrhinal, lateromedial, and laterointermediate, anteriorlateral, rostrolateral, anterior, anteromedial, and posterior medial cortex: P, POR, LM, LI, AL, RL, A, AM, PM (Wang and Burkhalter, 2007). Relating these areas to previous nomenclature of extrastriate visual areas, PM and AM (Wang and Burkhalter, 2007) are equivalent to area V2ML (Franklin and Paxinos, 2008) whereas V2L (Franklin and Paxinos, 2008) is broadly equivalent to areas RL, AL, LM, LI, P, and POR (Watson, 2012). However, this dissertation is going to focus on the lateral extrastriate cortex areas P, POR, LM and LI. Functional studies have shown that compared to neurons in V1, neurons in LI prefer higher spatial and temporal frequencies, whereas neurons in LM prefer up to three times faster temporal frequencies and significantly lower spatial frequencies than V1 (Marshel et al., 2011).

It is widely known that the response properties in the rodent visual cortex have a dispersed "salt and pepper" organization, which is very different from the columnar organization of the primate cortex. In monkeys, it has been reported that the motionselective MT area is topographically organized with the pulvinar projection (in which retrograde tracers filled cells in the pulvinar nucleus were arranged correspondingly 
with individual injection sites at different retinotopic locations in MT), with the lower visual field represented dorsally and the upper visual field represented ventrally (Standage and Benevento, 1983). Olavarria and Torrealba found that in rats, the receptive field properties in area 18 a were relatively slightly affected with destruction of striate cortex (Olavarria and Torrealba, 1978). With the removal of the whole striate cortex, tree shrews retain the ability to discriminate between different patterns and different colors, but additional ablation of extrastriate areas 18 and 19 rendered tree shrews unable to discriminate between upright and inverted triangles (Snyder et al., 1966; Snyder and Diamond, 1968). However, how the pulvinar influences cortical circuitry has not been explored. In all mammals, there are two main types of neurons in the neocortex, one type are the neurons which have spines on their dendrites and are excitatory (glutamatergic) and project both within and outside of the cortex, while the other type are neurons that have smooth dendrites, are inhibitory (GABAergic), and do not project outside of the cortex (interneurons). The experiments described in Chapter IV were designed to determine which types of cortical neurons are directly innervated by pulvinar and whether the pulvinar nucleus targets specific cortical output channels.

\section{Optogenetics and the mouse model}

Brain perturbation techniques have evolved from ablation or chemical lesions, to reversible chemical or cooling inactivation/electrical activation, to the current widely used optogenetic techniques. Optogenetics allows activation or inactivation of specific brain pathways using light. This enables experiments to address questions 
that could not be accurately addressed before. The pathways studied in this dissertation could not be preserved in slices and therefore had not previously been studied using in vitro preparations. Thus, we adopted an optogenetic approach combined with in vitro whole cell recordings to address our questions. Due to the expanding research carried out using mice, the convenience and availability of transgenetic mouse lines, and the lack of information regarding the mouse pulvinar nucleus, all experiment were designed to utilize this model species.

\section{Outline of Chapters II-V}

As a first step toward understanding the role that the SC plays in the tectogeniculate pathway, I carried out a detailed comparison of the ultrastructural characteristics of tectal input, cortical input, and retinal input in the dLGN (Chapter II). To further understand the tectopulvinar pathway, I reviewed previous studies and added evidence from current mouse studies (Chapter III). Finally, in Chapter IV, I investigated the ultrastructure and synaptic characteristics of pulvinocortical connections, and also explored the cortical output channels affected by the pulvinar nucleus. In Chapter V, I summarized my findings and discussed the direction of future studies related to the exploration of tectothalamocortical pathways. 


\section{CHAPTER II}

\section{RETINAL AND TECTAL “DRIVER-LIKE” INPUTS CONVERGE IN THE SHELL OF THE MOUSE DORSAL LATERAL GENICULATE NUCLEUS ${ }^{1}$}

\section{Overview}

The dorsal lateral geniculate nucleus ( $\mathrm{dLGN}$ ) is a model system for understanding thalamic organization and the classification of inputs as "drivers" or "modulators." Retinogeniculate terminals provide the primary excitatory drive for the relay of information to visual cortex (V1), while nonretinal inputs act in concert to modulate the gain of retinogeniculate signal transmission. How do inputs from the superior colliculus, a visuomotor structure, fit into this schema? Using a variety of anatomical, optogenetic, and in vitro physiological techniques in mice, we show that dLGN inputs from the superior colliculus (tectogeniculate) possess many of the ultrastructural and synaptic properties that define drivers. Tectogeniculate and retinogeniculate terminals converge to innervate one class of dLGN neurons within the dorsolateral shell, the primary terminal domain of direction-selective retinal ganglion cells. These dLGN neurons project to layer I of V1 to form synaptic contacts with dendrites of deeper-layer neurons. We suggest that tectogeniculate inputs act as

1. Bickford ME, Zhou N, Krahe TE, Govindaiah G, Guido W. J Neurosci 35:10523-10534. 2015. DOI: 10.1523/JNEUROSCI.3375-14.2015. 
"backseat drivers," which may alert shell neurons to movement commands generated by the superior colliculus.

\section{SIGNIFICANCE STATEMENT}

The conventional view of the dorsal lateral geniculate nucleus (dLGN) is that of a simple relay of visual information between the retina and cortex. Here we show that the dLGN receives strong excitatory input from both the retina and the superior colliculus. Thus, the dLGN is part of a specialized visual channel that provides cortex with convergent information about stimulus motion and eye movement and positioning.

\section{Introduction}

The concept of defining afferents as "drivers" or "modulators" has reshaped our current view of thalamic function. The dorsal lateral geniculate nucleus (dLGN), the principal relay of retinal information to the visual cortex, is widely accepted as a prototype for delineating the structural and functional organization of these two classes of inputs (Sherman and Guillery, 1998; Guillery and Sherman, 2002). While retinal inputs provide the primary excitatory drive onto thalamocortical (relay) cells in the dLGN, they comprise only about $10 \%$ of all synapses in this nucleus. Instead, the vast majority of synapses arise from nonretinal sources, such as projections from the visual cortex, brainstem, and thalamic reticular nucleus. These nonretinal inputs have been characterized as modulators because they have little impact on the receptive field structure of relay cells, but provide a powerful substrate for modulating the gain of retinogeniculate $(\mathrm{RG})$ signal transmission in a state-dependent manner. 
A projection from the superior colliculus (SC), or tectum, to the dLGN has been identified in a number of mammalian species (Harting et al., 1991), but remarkably, an understanding of how tectogeniculate (TG) input fits within the framework of thalamic function is lacking. In rodents, TG projections are confined to the dorsolateral shell, a thin lamina that resides just beneath the optic tract (OT; (Reese, 1988; Grubb and Thompson, 2004). This region is innervated by retinal ganglion cells that are sensitive to the direction of visual motion [direction-selective ganglion cells (DSGCs); Huberman et al., 2009; Kay et al., 2011; Rivlin-Etzion et al., 2011; Dhande et al., 2013; Cruz-Martín et al., 2014] and also contains dLGN cells that exhibit direction-selective responses (Marshel et al., 2012; Piscopo et al., 2013; Scholl et al., 2013; Zhao et al., 2013). Such convergence suggests that TG projections participate in coding the direction of visual stimulus movement. This idea is further supported by the fact that TG cells are located in the most superficial regions of the SC stratum griseum superficiale (SGS; Mooney et al., 1988; Diamond et al., 1991). Like the dorsolateral shell of the dLGN, the SGS is innervated by DSGCs (Huberman et al., 2009; Kay et al., 2011; Rivlin-Etzion et al., 2011; Dhande et al., 2013a) and contains neurons that respond selectively to visual motion, some of which have been identified as TG cells (Mooney et al., 1985, 1988; Waleszczyk et al., 2004; Wang et al., 2010; Gale and Murphy, 2014; Inayat et al., 2015). Thus, the dorsolateral shell of the dLGN appears to receive two distinct sources of direction-selective signals, one from the retina and one from the SC. Surprisingly, we found that TG inputs display the signature characteristics of driver synapses. Moreover, TG and retinal inputs 
converge to innervate a distinct class of relay cells that project to layer 1 of V1.

Together our results provide further evidence for structurally and functionally distinct parallel visual channels within the mouse dLGN.

\section{Materials and Methods}

\section{Animals}

All breeding and experimental procedures were approved by the University of Louisville and Virginia Commonwealth University Institutional Animal Care and Use Committees. Experiments were performed using mice, of either sex, of the following lines: C57/BLK6; mice that the express green fluorescent protein (GFP) in thyrotropin-releasing hormone receptor (TRHR)-expressing ganglion cells (RivlinEtzion et al., 2011); the Cre driver line GAD2-ires-Cre (Jackson Labs, stock \#010802); Gad2tm2(cre)Zjh/J (Taniguchi et al., 2011); and the GAD2-ires-Cre mice crossed with the Ai9 reporter line [Jackson Labs, stock \#007900, strain B6.CgGt(ROSA)26Sortm9(CAG-tdTomato)Hze/J].

\section{Biotinylated dextran amine injections}

To label TG or geniculocortical (GC) projections via anterograde transport, mice ranging in age between postnatal day 30 (P30) and P60 were deeply anesthetized with a mixture of ketamine $(120-140 \mathrm{mg} / \mathrm{kg})$ and xylazine $(12-14 \mathrm{mg} / \mathrm{kg})$ and placed in a stereotaxic apparatus (Angle Two Stereotaxic, Leica). An incision was made along the scalp, and a small hole was drilled in the skull above the SC or dLGN. A glass pipette (10 $\mu \mathrm{m}$ tip diameter) containing a 5\% solution of biotinylated dextran amine (BDA; Invitrogen) in saline was lowered into the SC or dLGN, and BDA was 
iontophoretically ejected using $3 \mu \mathrm{A}$ continuous positive current for $20 \mathrm{~min}$. After removal of the pipette, the scalp skin was sealed with tissue adhesive (n-butyl cyanoacrylate), and the animals were placed on a heating pad until mobile. After surgery, animals were carefully monitored for proper wound healing, and the analgesic buprenorphine $(0.02 \mathrm{mg} / \mathrm{kg})$ was administered every $12 \mathrm{~h}$ for $48 \mathrm{~h}$.

\section{Cholera toxin subunit B injections}

To label TG cells via retrograde transport, P30-P60 wild-type or GAD2-creAi9 mice were prepared as described above. A glass pipette (10-20 $\mu \mathrm{m}$ tip diameter) containing a $0.2 \%$ solution of cholera toxin subunit B (CTB) conjugated to Alexa Fluor 488 (CTB-488) in PBS [0.01 m phosphate buffer (PB) with $0.9 \% \mathrm{NaCl}$ ] was lowered into the dLGN, and CTB was iontophoretically ejected using $3 \mu \mathrm{A}$ continuous positive current for $15 \mathrm{~min}$. After removal of the pipette, the wound was closed and the animals were treated during recovery as described above.

\section{Adeno-associated virus and CTB injections}

An adeno-associated virus (AAV; serotype 2/1) carrying a vector for the Channelrhodopsin variant Chimera EF with I170 mutation (ChIEF) fused to the red fluorescent protein, tdTomato, was injected into the SC of wild-type mice to label TG terminal arbors in the dLGN for light and electron microscopic analysis as well as to photoactivate TG terminals during in vitro physiology experiments (for production details, see Jurgens et al., 2012). For virus delivery, mice ranging in age between P22 and P24 were deeply anesthetized with a mixture of ketamine and xylazine. An incision was made along the scalp, and a small hole created in the skull above the SC. 
Virus was delivered via a 34 gauge needle attached to a Nanofil syringe inserted in an UltraMicroPump. Volumes of 100-200 $\mathrm{nl}$ were injected at a rate of $10 \mathrm{nl} / \mathrm{min}$.

In some animals, following virus injections in the SC, an additional hole was drilled in the skull above V1. The dura was carefully removed, and a small piece of filter paper that had been immersed in a $0.1 \%$ solution of CTB-488 in PBS was placed on the cortex surface to label geniculocortical cells by retrograde transport. The wound was then closed and the animal monitored during recovery as described above.

\section{Cre-dependent AAV injections in GAD2-Cre mice}

Flex-rev-oChIEF-tdTomato (plasmid 30541, Addgene) was packaged using AAV serotype 9. This viral vector was injected into the SC of GAD2-cre mice to examine GABAergic projections from this structure. For virus delivery, mice ranging in age between P25 and P36 were deeply anesthetized with a mixture of ketamine and xylazine. An incision was made along the scalp, and a small hole created in the skull above the SC. Virus was delivered via a 34 gauge needle attached to a Nanofil syringe inserted in an UltraMicroPump. Volumes of 60-75 $\mathrm{nl}$ were injected at a rate of 10-20 $\mathrm{nl} / \mathrm{min}$.

\section{Slice preparation and in vitro recording}

At 8 to $12 \mathrm{~d}$ following injection of the AAV serotype $2 / 1$, mice were deeply anesthetized with Avertin $(0.5 \mathrm{mg} / \mathrm{gm})$, rapidly decapitated, and brains were placed into cold $\left(4^{\circ} \mathrm{C}\right)$, oxygenated $(95 \% \mathrm{O} 2 / 5 \% \mathrm{CO} 2)$ slicing solution containing the following (in mm): $2.5 \mathrm{KCl}, 26 \mathrm{NaHCO} 3,2.5 \mathrm{KCl}, 1.25 \mathrm{NaH} 2 \mathrm{PO} 4,10 \mathrm{MgCl} 2,2$ $\mathrm{CaCl} 2,234$ sucrose, and 11 glucose. Coronal slices $(300 \mu \mathrm{m})$ were cut at the level of 
dLGN and SC using a vibrating tissue slicer (Leica). Then slices were incubated in oxygenated (95\% O2/5\% CO2) artificial CSF (ACSF) [containing (in mm) $126 \mathrm{NaCl}$, $26 \mathrm{NaHCO} 3,2.5 \mathrm{KCl}, 1.25 \mathrm{NaH} 2 \mathrm{PO} 4,2 \mathrm{MgCl} 2,2 \mathrm{CaCl} 2$, and 10 glucose] at $32^{\circ} \mathrm{C}$ for $30 \mathrm{~min}$ and later maintained at room temperature.

Whole-cell recordings were obtained from dLGN neurons as described previously with minor modifications (Govindaiah et al., 2012). Briefly, individual brain slices were transferred to a recording chamber that was maintained at $32^{\circ} \mathrm{C}$ and continuously perfused with oxygenated ACSF $(2.5 \mathrm{ml} / \mathrm{min}, 95 \% \mathrm{O} 2 / 5 \% \mathrm{CO} 2)$. Neurons were visualized on an upright microscope (Olympus BX51WI) equipped with both differential interference contrast optics and filter sets for visualizing CTB488 (Chroma 49002) or tdTomato (Chroma 49005) using a $10 \times$ or $60 \times$ waterimmersion objective (Olympus) and a CCD camera. Recording pipettes were pulled from borosilicate glass capillaries using a vertical puller (Narishige) and filled with an intracellular solution containing the following (in mm): $117 \mathrm{~K}$-gluconate, $13.0 \mathrm{KCl}, 1$ $\mathrm{MgCl} 2,0.07 \mathrm{CaCl} 2,0.1$ EGTA, 10 HEPES, $2 \mathrm{Na}-\mathrm{ATP}$, and $0.4 \mathrm{Na}-\mathrm{GTP}$. The pH and osmolality of internal solution were adjusted to 7.3 and $290 \mathrm{mOsm}$, respectively. Whole-cell recordings were obtained using a Multiclamp 700B amplifier (Molecular Devices). Data were filtered at $2.5 \mathrm{kHz}$, digitized at $10 \mathrm{kHz}$, and analyzed using pClamp 10 (Molecular Devices). In some experiments, data acquisition and analysis were accomplished using Strathclyde Electrophysiology Software (Whole Cell Analysis Program, version 3.8.2), and digitized at 10-100 kHz through an analog-todigital board (National Instruments, PCI-6221). A $10 \mathrm{mV}$ junction potential was 
subtracted for all voltage recordings. For voltage measurements, recordings were made from cells with a resting membrane potential between -55 and $-75 \mathrm{mV}$, whereas membrane currents were obtained at $-70 \mathrm{mV}$. Pipette capacitance, series resistance, and whole-cell capacitance were carefully monitored and compensated electronically during the recording. Only experiments in which series resistance remained relatively stable (20\% change) were included for analysis.

For photoactivation of tectogeniculate terminals, light from a blue light emitting diode (Prizmatix UHP 460) was reflected into a $4 \times$ or $60 \times$ objective. This produced a spot of blue light onto the submerged slice with an approximate diameter of 2.2 or $0.45 \mathrm{~mm}$, respectively. Pulse duration and frequency were under computer control. For repetitive stimulation, pulse duration was between 2 and $7 \mathrm{~ms}$.

For electrical activation of retinogeniculate terminals, a monopolar electrode was placed in the OT. Synaptic responses were evoked with various intensities (25$150 \mu \mathrm{A})$ and frequencies $(1-100 \mathrm{~Hz})$ at $10 \mathrm{~s}$ interstimulus intervals. EPSCs were evoked at a holding potential of $-70 \mathrm{mV}$ in the presence of the $\mathrm{GABA}_{\mathrm{A}}$ receptor $\left(\mathrm{GABA}_{\mathrm{A}} \mathrm{R}\right)$ antagonist SR95531 (4-[6-imino-3-(4-methoxyphenyl) pyridazin-1-yl] butanoic acid hydrobromide; $10 \mu \mathrm{m})$.

The peak amplitude of synaptic responses was measured from baseline values for three to five traces for each condition using pClamp 10 software (Molecular Devices). To quantify the degree of facilitation in responses evoked by repetitive trains of light or electrical stimulation, the amplitude of the second to tenth responses was divided by the amplitude of the initial response. The paired-pulse ratio (PPR) was 
determined by dividing the amplitude of the second EPSC by the amplitude of the first EPSC.

\section{Histology}

At the conclusion of in vitro recordings, dLGN slices were placed in a fixative solution of $4 \%$ paraformaldehyde in $0.1 \mathrm{~m} \mathrm{~PB}$. Slices were kept in this solution overnight at $4{ }^{\circ} \mathrm{C}$ and then washed in PB. For labeling, slices were treated with $0.1 \%$ Triton X-100 and Alexa Fluor 633 conjugated to strepavidin (1:1000; Invitrogen, catalog no. S21374) in PBS for 24 h. Slices were then rinsed with PBS, mounted with Prolong Gold (Invitrogen, catalog no. P36930), and coverslipped. Images of filled cells were collected using a confocal microscope (Olympus, model no. FV1200BX61).

Two days following injection of CTB-488, $7 \mathrm{~d}$ following injection of BDA, 10 $\mathrm{d}$ following the injection of AAV 2/1 or AAV 2/1 and CTB-488, or 10-16 d after the injection of cre-dependent AAV9, mice were deeply anesthetized with Avertin (0.5 $\mathrm{mg} / \mathrm{gm}$ ) and transcardially perfused with a fixative solution of $4 \%$ paraformaldehyde in $0.1 \mathrm{~m} \mathrm{~PB}, 2 \%$ paraformaldehyde and $2 \%$ glutaraldehyde in $0.1 \mathrm{~m} \mathrm{~PB}$, or $4 \%$ paraformaldehyde and $2.5 \%$ glutaraldehyde in $0.1 \mathrm{~m}$ cacodylate buffer. Brains were removed from the skulls and 70- $\mu \mathrm{m}$-thick coronal sections of cortex and thalamus were cut using a vibratome (Leica). Sections that contained tdTomato and/or CTB488 were mounted on slides and imaged using a confocal microscope (Olympus FV1200BX61). 
Sections that contained CTB-488 injections in the dLGN and CTB-488labeled TG cells were incubated overnight in a rabbit anti-CTB antibody (Sigma, catalog \#C3062; 1:10,000). For wild-type animals that received CTB injections, the following day, the sections were incubated in a 1:100 dilution of a biotinylated goatanti-rabbit antibody $(1 \mathrm{~h})$ followed by a 1:100 dilution of avidin and biotinylated horseradish peroxidase (ABC solution, Vector Laboratories; $1 \mathrm{~h}$ ) and reacted with nickel-enhanced diaminobenzidine (DAB). The sections were then mounted on slides, and a Neurolucida system (MBF Bioscience) was used to plot the distribution of the CTB-labeled TG cells. The MBF Bioscience solid modeling extension module was then used to reconstruct the SC and view the distribution of TG cells across its dorsal surface. For GAD2-cre-Ai9 animals that received CTB injections, sections were incubated overnight in the rabbit anti-CTB antibody $(1: 10,000)$, and the following day the sections were incubated in a 1:100 dilution of a biotinylated goat-anti-rabbit antibody $(1 \mathrm{~h})$, followed by a 1:100 dilution of avidin conjugated to Alexa Fluor 488 (Vector Laboratories). The sections were then mounted on slides and imaged using a confocal microscope.

For ultrastructural analysis of tdTomato-labeled terminals following virus injections, the sections were incubated in a 1:1000 dilution of a rabbit-anti-DsRed antibody (Clonetech, catalog \#632496) overnight. The following day, the sections were incubated in a biotinylated goat-anti-rabbit antibody, $\mathrm{ABC}$ solution, and reacted with DAB (as described above). To reveal the location of BDA, sections were incubated overnight in $\mathrm{ABC}$ solution and reacted the next day with $\mathrm{DAB}$. 
For electron microscopy, sections that contained DAB-labeled terminals were postfixed in $2 \%$ osmium tetroxide, dehydrated in an ethyl alcohol series, and flat embedded in Durcupan resin between two sheets of Aclar plastic (Ladd Research). Durcupan-embedded sections were first examined with a light microscope to select areas for electron microscopic analysis. Selected areas were mounted on blocks, ultrathin sections were cut using a diamond knife, and sections were collected on Formvar-coated nickel slot grids. Selected sections were stained for the presence of GABA, as described previously (Chomsung et al., 2010). Briefly, we used a rabbit polyclonal antibody against GABA (Sigma, catalog \#A2052; used at a dilution of 1:1000-1:2000) that was tagged with a goat-anti-rabbit antibody conjugated to $15 \mathrm{~nm}$ gold particles (GE Healthcare). The sections were air dried and stained with a $10 \%$ solution of uranyl acetate in methanol for $30 \mathrm{~min}$ before examination with an electron microscope.

\section{Results}

\section{Organization of the dorsolateral shell}

Figure 1 depicts the structural composition of the dorsolateral shell of the dLGN in a mouse line (TRHR) that expresses GFP in DSGCs that respond to posterior motion (Rivlin-Etzion et al., 2011). GFP-labeled retinogeniculate projections in these mice terminate in a thin band that corresponds to the dorsolateral shell (Fig. 1A-F, green). To examine the relationship between DSGC terminal domains and TG input, virus injections were placed in the SC (Fig. 1C, inset) to induce the expression of TdTomato (Fig. 1A-D, red) in TG terminals $(n=2)$. As expected, TG projections 
terminate in the dorsolateral shell and overlap with DSGC projections throughout the rostrocaudal extent of the dLGN (Fig. 1A-C). To quantify the distribution of TG terminals relative to TRHR terminals, we calculated the number of red pixels contained within the region of GFP-labeled terminals and divided this by the number of red pixels within the borders of the dLGN ( $n=5$ sections). This analysis revealed that $95.67 \pm 3.7 \%$ of labeled tectogeniculate terminals were located in the dorsolateral shell, as defined by the presence of GFP-labeled TRHR terminals.

To examine the type of dLGN cells that receive input from these two sources, we made in vitro whole-cell recordings in the dorsolateral shell of TRHR mice and filled recorded cells with biocytin (Fig. 1E, F). Confocal reconstructions of biocytinfilled cells revealed that relay neurons residing in GFP-labeled terminal domains $(\mathrm{n}=$ 12) had a hemispheric dendritic architecture resembling "W-like" cells (Krahe et al., 2011). In fact, recordings restricted to this region in wild-type mice, showed that all filled cells $(\mathrm{n}=32)$ were W-like (Figs. 1, 4, 7). Using our previously reported Scholl ring analysis (Krahe et al., 2011), all filled cells in the dorsolateral shell $(\mathrm{n}=12$ in TRHR and $n=32$ in wild type) exhibited direction of orientation index values of $0.5-$ 0.79. The overlap of tectal terminals and W-like cells in the mouse is consistent with the pattern seen in the carnivore $\mathrm{C}$ laminae and the primate koniocellular layers of the dLGN (Stanford et al., 1981; Harting et al., 1991; Lachica and Casagrande, 1993), suggesting that across species, the tectogeniculate pathway is a component of a distinct visual channel. 


\section{Tectogeniculate topography}

To examine the distribution of TG cells, small iontophoretic injections of the retrograde tracer CTB were confined to different regions of the dLGN in wild types (n $=8$ ). These injections labeled cells in restricted regions of V1 and the SGS of the SC. Following injections in the lateral dLGN (Fig. 2A, injection site photo; F, injection site reconstructed in red), corticogeniculate (CG) cells were labeled in rostral V1 (A), and TG cells were confined to lateral regions of the SGS (D, labeled TG cells, red arrow; G, full distribution of TG cells, red dots), whereas injections in the medial dLGN (C, injection site photo; F, injection site reconstructed in green) labeled CG cells in caudal V1 (B) and TG cells in the medial regions of the SGS (E, labeled cells, green arrow; $\mathrm{G}$, full distribution of TG cells, green dots). These results indicate that TG projections are organized in a topographic manner. Comparison of the distribution of TG cells labeled by retrograde transport to the organization of visual receptive fields in the mouse SGS (Dräger and Hubel, 1976) suggests that TG projections to the medial or lateral dLGN represent the upper/nasal and lower/temporal visual fields, respectively (Fig. 2G). Together, these results indicate that $\mathrm{CG}$ and TG projections are in register with the visual field representation in V1 (Dräger, 1975) and the dLGN (Piscopo et al., 2013).

\section{The vast majority of tectogeniculate projections are non-GABAergic}

To determine whether TG projections contain GABA, we performed two different experiments. First, we iontophoretically injected CTB-488 into the dLGN of GAD2-cre-Ai9 mice (in which tdTomato is expressed in GABAergic neurons) to label 
TG cells by retrograde transport, as described and illustrated above $(n=3)$. A one in two series of sections from each case was mounted on slides, and all SC sections that contained CTB-labeled cells were imaged using a confocal microscope using $2 \mu \mathrm{m}$ optical sections (Fig. 3A). In each imaged section, the number of CTB-labeled cells within the SGS that did (Fig. 3B, C, arrow) or did not (asterisks) contain TdTomato were counted. This analysis revealed that very few TG cells contained TdTomato (case 1, 19 of 497 TG cells, or $3.82 \%$; case 2,36 of 629 TG cells, or 5.72\%; case 3, 9 of $226 \mathrm{TG}$ cells, or $3.98 \%$; three cases combined, 64 of $1352 \mathrm{TG}$ cells, or $4.73 \%$ ), indicating that $95 \%$ of TG cells are non-GABAergic cells.

Next, we injected a cre-dependent virus in the SC of GAD2-cre mice $(n=8$; Fig. 3D) to label GABAergic cells in the SC and their axons projections with the red fluorescent protein tdTomato. The tdTomato-labeled axon projections of GABAergic SC neurons were densely distributed within the ventral lateral geniculate nucleus and pretectum. Sparse projections could also be detected in the dLGN (Fig. 3E), as described previously (Gale and Murphy, 2014). Together, these experiments demonstrate that a small population of TG cells are GABAergic and sparsely innervate the dLGN.

\section{TG terminals: a new class of dLGN synaptic profile}

The majority of cells in the mouse SGS prefer relatively small stimuli $\left(6-10^{\circ}\right)$, although this varies widely with cell type (Wang et al., 2010; Gale and Murphy, 2014). In the dLGN, direction-selective cells prefer larger spot sizes $\left(17.2 \pm 3.66^{\circ}\right.$; Piscopo et al., 2013), potentially due to convergence of retinogeniculate and/or TG 
inputs on their widespread dendritic arbors (Fig. 1F; Krahe et al., 2011). To determine how TG projections are distributed on the dendritic arbors of the $\mathrm{W}$-like cells in the dorsolateral shell, we next examined the ultrastructure of TG synaptic terminals and their postsynaptic dendrites and compared these projections to those formed by RG and CG projections.

Four major types of terminals have been found to make up the neuropil of the dLGN (Guillery, 1969; Bickford et al., 2010): (1) large glutamatergic terminals that contain round vesicles and distinctive mitochondria with widened cristae that make them appear pale relative to surrounding mitochondria (RLP profiles), identified as RG terminals (Robson and Mason, 1979; Fig. 4G,J,K, red); (2) small glutamatergic terminals with round vesicles and dark mitochondria (RS profiles; Fig. 4G, blue), which primarily originate from the cortex (Erisir et al., 1997); (3) profiles that contain GABA within densely packed flattened or pleomorphic vesicles (F1 profiles), contributed by the thalamic reticular nucleus, pretectum, or interneuron axons (Wang et al., 2001, 2002); and (4) interneuron dendritic terminals (Hamos et al., 1985) that contain GABA and loosely packed vesicles (F2 profiles).

To examine the ultrastructure of TG terminals, we first placed injections of BDA in the mouse SC to label terminals by anterograde transport, and then stained dLGN sections containing labeled terminals with an antibody against GABA. We found that three types of terminals were labeled: (1) RLP profiles, (2) F1 profiles, and (3) non-GABAergic profiles that contained round vesicles and dark mitochondria but that were larger than RS profiles (RM profiles; Fig. 4A-F, J, K). 
We interpret the BDA-labeled RLP profiles as retinal terminals originating from axons that branch to innervate both the dLGN and SC (Tamamaki et al., 1995); we demonstrated previously in cats that SC BDA injections can be used to label the geniculate branches of retinotectal axons (Datskovskaia et al., 2001). The BDAlabeled F1 profiles originate either from the SC (based on the results described above) or from other sources, such as GABAergic pretectal cells that innervate the dLGN (Cucchiaro et al., 1991; Bickford et al., 2000; Wang et al., 2002) and SC (Baldauf et al., 2003). We interpret the BDA-labeled RM profiles as TG terminals that originate from the non-GABAergic TG cells described above.

To quantify TG terminal morphology without the complications of uptake by fibers of passage, we injected an AAV into the SC to induce the expression of the red fluorescent protein tdTomato in SC neurons and their axon projections (Fig. 1A-D). Using an antibody to detect the tdTomato, we then examined the ultrastructure of the virus-labeled terminals. It is important to note that none of the virus-labeled TG terminals contained pale mitochondria, indicating that TG terminals can be distinguished from $\mathrm{RG}$ profiles based on the ultrastructure of their mitochondria (Robson and Hall, 1977). We also found that virus-labeled TG terminals were significantly smaller than RLP profiles (Fig. 4H; one way ANOVA, p < 0.0001). Moreover, TG terminals were significantly larger than CG terminals, which we labeled by injecting BDA in V1 (Fig. 3G; one way ANOVA, p < 0.001). Thus, TG terminals constitute a new class of dLGN synaptic profile. 
Finally, we examined the size of the dendrites postsynaptic to RG terminals (identified by their ultrastructure; $n=179)$, virus-labeled TG terminals $(n=108)$, and BDA-labeled CG terminals $(\mathrm{n}=87)$. As described previously for other species (Wilson et al., 1984; Li et al., 2003a), mouse RG terminals contact significantly larger (more proximal) dendrites compared to the dendrites contacted by CG terminals (Fig. 4I; one way ANOVA, $p<0.0001)$. Surprisingly, we detected no significant difference in the size of dendrites contact by TG and RG terminals. This suggests that RG and TG terminals both innervate the more proximal dendrites of dLGN neurons. In fact, we noted that in some cases TG terminals contacted dendrites that were also contacted by RLP profiles (Fig. 4J, K), indicating convergence of TG and RG inputs onto single neurons of the dorsolateral shell.

\section{TG responses are "driver-like" and converge with RG inputs}

To examine the postsynaptic responses in the dLGN elicited by activation of TG inputs, we injected an AAV carrying the coding sequence for TdTomato and the channel rhodopsin variant Chimera EF with I170 mutation (Jurgens et al., 2012). This induced the expression of TdTomato/ChIEF in TG terminals within the dorsolateral shell (Fig. 1A-C). Whole-cell recordings of dLGN cells were obtained with biocytinfilled pipettes within regions of the dorsolateral shell that contained labeled TG terminals (Fig. 5A). Blue light stimulation of these regions (1-100 ms duration) evoked large and reliable EPSPs that were capable of eliciting trains of action potentials that faithfully followed the duration and temporal frequency of stimulation (Fig. 5B-D). At hyperpolarized membrane potentials, light-activated EPSPs could 
also evoke low-threshold $\mathrm{Ca}^{2+}$ spikes and burst firing (Fig. 5B). These light-evoked responses were unaffected by bath application of the $\mathrm{GABA}_{\mathrm{A}}$ receptor antagonist bicuculline $(10 \mu \mathrm{m})$, were reduced in amplitude by bath application of the NMDA antagonist AP5 $(50 \mu \mathrm{m})$, and completely abolished with the subsequent addition of the AMPA receptor antagonist DNQX (10 $\mu \mathrm{m}$; Fig. 5C, D). Gale and Murphy (2014) demonstrated previously that inhibitory postsynaptic potentials can be elicited in dLGN cells when the GABAergic TG projections are isolated for activation using optogenetic techniques in transgenic animals. However, our experimental protocols did not detect this minor projection. Reconstructions of biocytin-filled cells that were activated by blue light $(\mathrm{n}=32)$ indicated they all displayed W-like morphology (Fig. 5A; dendritic orientation index, 0.5-0.79; Krahe et al., 2011). Together, our anatomical and physiological results indicate that the vast majority of TG terminals are glutamatergic and contact the proximal dendrites of $\mathrm{W}$ cells in the dorsolateral shell of the dLGN.

Voltage-clamp recordings to pairs (Fig. 6A) or trains (B) of repetitive pulses of blue light revealed a strong synaptic depression. Paired-pulse stimulus presentation (100 ms interstimulus interval) showed an approximately twofold reduction in EPSC amplitude between the initial (EPSC1) and second response (EPSC2; PPR mean, $0.56 ; \mathrm{n}=15$; Fig. $6 \mathrm{~A}$ ). A similar form of paired-pulse depression was observed with a $20 \mathrm{~Hz}$ train of light, with response amplitudes of subsequent EPSCs showing no further reduction (Fig. 6B; $n=12$ ). To compare this feature of TG responses to those of simultaneously recorded retinogeniculate synaptic responses, we electrically 
stimulated the OT to activate RG terminals. We found that neurons that exhibited a light-evoked TG response were also activated by electrical stimulation of the OT (Fig. $7 ; \mathrm{n}=6$ ). Both sets of responses were large in amplitude (mean $\pm \mathrm{SEM} ; \mathrm{RG}=601 \pm$ $58.8 \mathrm{pA} ; \mathrm{TG}=238 \pm 34.1 \mathrm{pA}$, both $\mathrm{n}=6$; paired $\mathrm{t}$ test indicates significant difference at $p=0.002)$, blocked by glutamate antagonists $(n=3$; Fig. 6 , inset $)$, and showed paired-pulse depression. At shorter interstimulus intervals (50 ms vs $500 \mathrm{~ms}$ ), PPR values reflected a greater form of depression for TG to compared to RG stimulation (paired t test, $\mathrm{p}<0.001$ ). Such excitatory convergence is consistent with our ultrastructural results (Fig. 4J, K). The differences in overall EPSC amplitudes and PPRs of these two terminal types may reflect the smaller terminal profiles of TG input (Fig. $4 \mathrm{H}$ ) and/or the complement of presynaptic proteins that regulate the synaptic vesicle cycle (Kielland et al., 2006; Wei et al., 2011).

\section{Neurons postsynaptic to TG terminals contact non-GABAergic dendrites in V1}

\section{layer I}

In primates, TG projections are confined to the koniocellular layers of the dLGN (Harting et al., 1991), which project to the superficial layers of V1 (Hendry and Yoshioka, 1994; Casagrande et al., 2007). Similarly, W cells in the tectorecipient layers of the cat dLGN project primarily to the superficial layers of V1 (Anderson et al., 2009). To test whether a similar organization is found in the mouse, we combined retrograde tracing techniques with viral vector injections. To label dLGN cells that project to layer I, we placed small pieces of filter paper infused with CTB-488 on the surface of V1 (Fig. 8A); in the same animals, we placed virus injections in the SC 
(Fig. 8B) to label TG terminals. In cases in which there was no damage to the cortex surface and the placement of the tracer was confined to the most superficial layers (Fig. 8A), dLGN cells labeled by retrograde transport were confined to the dorsolateral shell, overlapping the distribution of TG terminals (Fig. 8C). This corresponds with a previous study in which tracer or virus injections confined to the superficial layers of V1 labeled cells in the dorsolateral shell (Cruz-Martín et al., 2014). To determine whether layer I projecting cells receive direct input from the SC, we repeated these dual tracing experiments and then prepared the tissue for acute in vitro electrophysiology. Using only cases in which we confirmed that the cortex was not damaged by our CTB placement, we targeted our recordings to CTB-488-labeled dLGN cells using epifluorescence to guide the placement of our pipettes and then activated the TG terminals using blue light pulses. As expected, layer I projecting cells displayed W-like morphology (Fig. 8D) and responded to light activation of TG input (E).

Finally, to identify the V1 synaptic targets of TG-recipient neurons, we placed BDA injections in the dLGN (Fig. 9A, inset) to label geniculocortical terminals (A) and examined the layer I projections (A, arrows; B) in tissue processed for electron microscopy. This tissue was also stained with an antibody against GABA. Although layer I contains a high density of GABAergic neurons (Ma et al., 2014), virtually all dLGN projections within layer I of V1 contacted non-GABAergic dendrites ( 85 of 87 synaptic contacts, or 98\%; Fig. 9C). Thus, TG projections likely influence the activity 
of lower-layer excitatory spiny cells, which extend their apical dendritic tufts within layer I of V1.

\section{Discussion}

The mouse dLGN contains at least two functional subdivisions: the core and its surrounding dorsolateral shell (Dhande and Huberman, 2014). Our results, combined with those of previous studies, indicate that the circuits formed by these two zones are fundamentally different (Fig. 10). Within the core, neurons exhibit Xor Y-like morphology (i.e., biconical or symmetrical dendritic fields; Krahe et al., 2011) and project to layer IV of V1, whereas neurons in the shell exhibit W-like morphology (hemispheric dendritic arbors) and project to layer I of V1 (Krahe et al., 2011; Cruz-Martín et al., 2014). Moreover, although both core and shell neurons receive cortical input on their distal dendrites, the innervation of their proximal dendrites is quite distinct. The proximal dendrites of core neurons are innervated by non-direction-selective ganglion cells (Huberman et al., 2008; Bickford et al., 2010; Kim et al., 2010; Kay et al., 2011), which drive center-surround receptive field properties (Piscopo et al., 2013). In contrast, the proximal dendrites of shell neurons receive convergent input from both direction-selective ganglion cells (Huberman et al., 2009; Kay et al., 2011; Rivlin-Etzion et al., 2011; Dhande et al., 2013; CruzMartín et al., 2014) and the SC. Presumably, the integration of these two inputs underlies emergent direction-selective properties of dorsolateral shell neurons (Marshel et al., 2012; Piscopo et al., 2013; Scholl et al., 2013; Zhao et al., 2013). 
The concept of driver inputs was defined based on the properties of RG terminals in regions homologous to the core of mouse dLGN (i.e., the A laminae of the cat dLGN; Sherman and Guillery, 1998). Inputs that can be classified as drivers are (1) large terminal profiles that synapse on proximal dendrites, (2) inputs that elicit fast primarily ionotropic glutamate transmission, and (3) inputs that when stimulated repetitively exhibit a high probability of glutamate release and synaptic depression (Guillery and Sherman, 2002; Li et al., 2003a, b). TG inputs exhibit many features of driver terminals. We found that these inputs are relatively large profiles that are located on proximal regions of the dendrites and display robust, fast excitatory transmission and synaptic depression. The similarities between RG and TG inputs suggest that in the dorsolateral shell, two driver-like inputs converge on single thalamocortical relay cells.

However, we use the term "driver-like" to describe converging TG and RG inputs with the proviso that such convergence contradicts the idea that each thalamic neuron is driven by one source of input, while all remaining inputs modulate this primary response (Sherman and Guillery, 1998). Similar examples of driver-like convergence illustrate the complexity of thalamic organization. The dorsal thalamus may be divided not only into "first order" (driven by ascending peripheral input) or "higher order" (driven by descending cortical input) nuclei (Guillery and Sherman, 2002), but also “second order" nuclei driven by convergent, ascending inputs (Kelly et al., 2003; Smith et al., 2007; Chomsung et al., 2008; Masterson et al., 2009, 2010), or nuclei that contain unique combinations of ascending and descending driver-like 
inputs (Baldauf et al., 2005; Rovo et al., 2012; Groh et al., 2014). Such diversity may dramatically increase the computational capabilities of the thalamus, reflecting its essential roles in sensory, motor, and sensory-motor circuits.

In the dLGN shell, the convergence of RG and TG inputs may be necessary to calculate the trajectory of visual stimuli in relation to movement of the eyes. In vitro studies of the SC indicate that tectothalamic cells in the superficial layers of the SC are disynaptically inhibited by deeper-layer premotor cells that innervate intrinsic GABAergic interneurons (Phongphanphanee et al., 2011). This circuit has been proposed to underlie "saccadic suppression," the attenuation of visual signals that occurs during the execution of eye or body movements to prevent blurring of the visual field. Alternatively, we suggest that this circuit could be used to adjust TG signals to reflect the direction and amplitude of impending body movements. In other words, TG inputs could be thought of as "backseat drivers" that alert shell neurons to movement commands generated by the superior colliculus. The resultant changes in the amplitude and/or timing of TG inputs could alter the response properties of dorsolateral shell neurons to reflect the contributions of self-generated and externally generated movement (for discussion of similar circuits, see Sommer and Wurtz, 2008). Thus, while silencing TG inputs in anesthetized animals may have relatively subtle effects on the receptive field properties of dLGN neurons (Xue et al., 1994), we predict that in behaving animals, TG inputs may strongly influence the manner in which neurons in the dorsolateral shell respond to moving visual stimuli. 
Using calcium imaging techniques, Cruz-Martín et al. (2014) demonstrated that geniculocortical axons in the superficial layers of mouse V1 exhibit directionselective responses, and our study indicates that these axons synapse on nonGABAergic dendrites. This arrangement suggests that tectorecipient dorsolateral shell neurons target the apical dendrites of neurons located in the deeper cortical layers of V1 (Fig. 10). Thus, V1 neurons may inherit direction selectivity not only via DSGCs (Cruz-Martín et al., 2014), but also from a driver-like circuit that originates in the SC. 


\section{Figure 1. Components of the dLGN dorsolateral shell}

In TRHR mice, in which GFP is expressed in direction-selective retinogeniculate terminals (green), virus injections were placed in the SC (inset in panel C) to induce the expression of TdTomato in tectothalamic terminals (red). A caudal (A) to rostral (C) series of sections illustrates the overlap of tectogeniculate and TRHR retinogeniculate terminals in the dorsolateral shell of the dLGN (also shown at higher magnification in panel D). Cells filled with biocytin in TRHR animals exhibit "W cell" morphology (E, arrow indicates cell shown at higher magnification in F). Scale bars: $\mathrm{C}=100 \mu \mathrm{m}$ and also applies to $\mathrm{A}$ and $\mathrm{B}, \mathrm{D}=20 \mu \mathrm{m}, \mathrm{E}=100 \mu \mathrm{m}, \mathrm{F}=25 \mu \mathrm{m}$. 


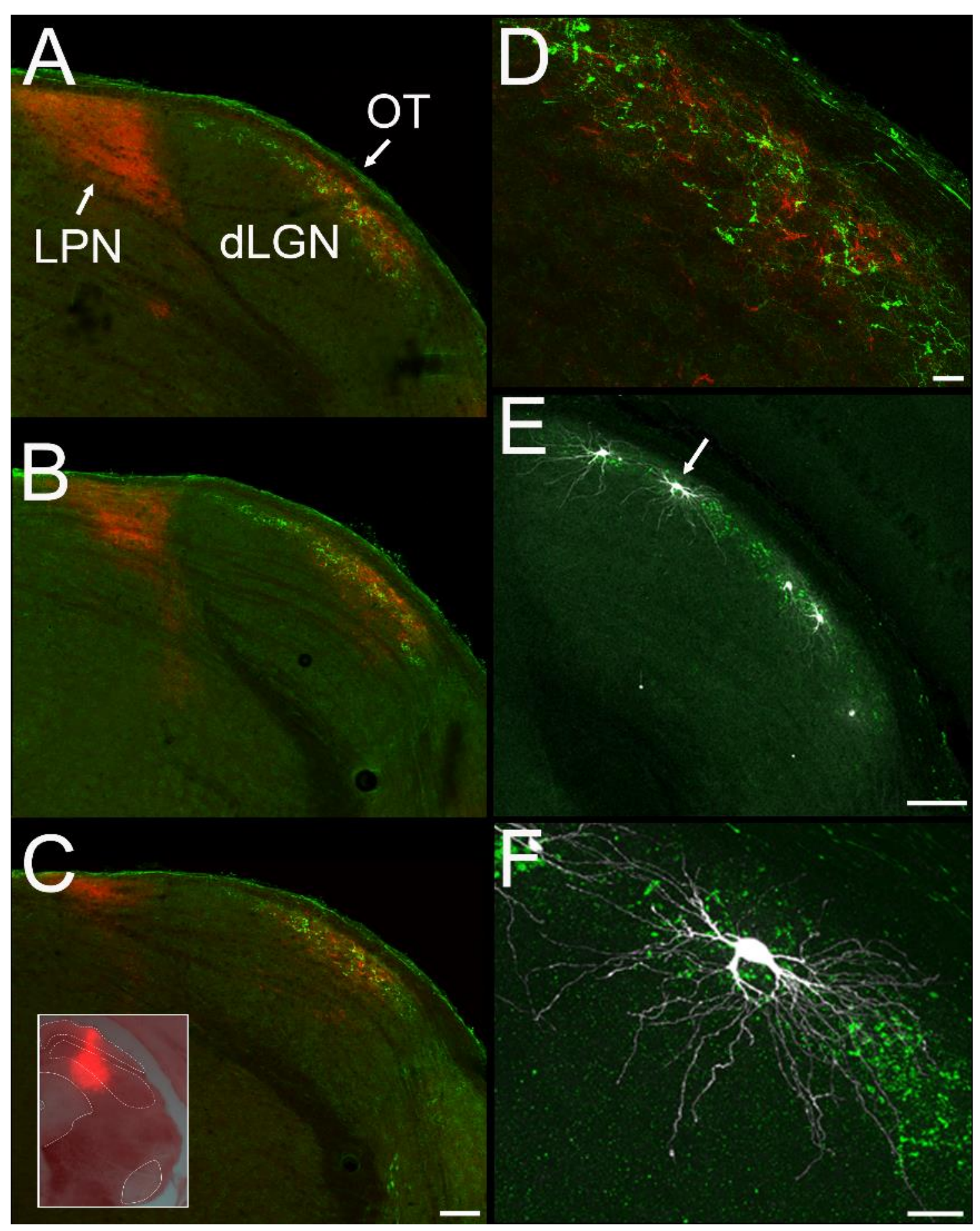




\section{Figure 2. Tectogeniculate (TG) topography}

Small iontophoretic injections of CTB in the lateral dLGN (A, coronal section, F, 3D reconstruction of dLGN, red) labeled corticogeniculate cells in layer VI of rostral V1 and the lateromedial (LM) cortex (A), and TG cells in the lateral stratum griseum superficiale (SGS) of the SC (D, coronal section, red arrow, G, 3D distribution, red dots). Small iontophoretic injections of CTB in the medial dLGN (C, coronal section, $\mathrm{F}, 3 \mathrm{D}$ reconstruction of $\mathrm{dLGN}$, green) labeled corticogeniculate cells in layer VI of caudal V1 (B) and TG cells in the medial SGS (E, coronal section, green arrow, G, 3D distribution, green dots). Based on the SC receptive field positions mapped by Dräger and Hubel (1976), TG projections to the medial and lateral dLGN likely represent upper and visual fields respectively (schematically indicated in G). Scale bars: $A=$ $500 \mu \mathrm{m}$ and applies to $\mathrm{B}$ and $\mathrm{C} . \mathrm{D}=250 \mu \mathrm{m}$ and also applies to E. Orientation of 3D reconstructions in $\mathrm{F}$ and $\mathrm{G}$ indicated by arrows, $\mathrm{D}$, dorsal, $\mathrm{L}$, lateral, $\mathrm{C}$, caudal. 


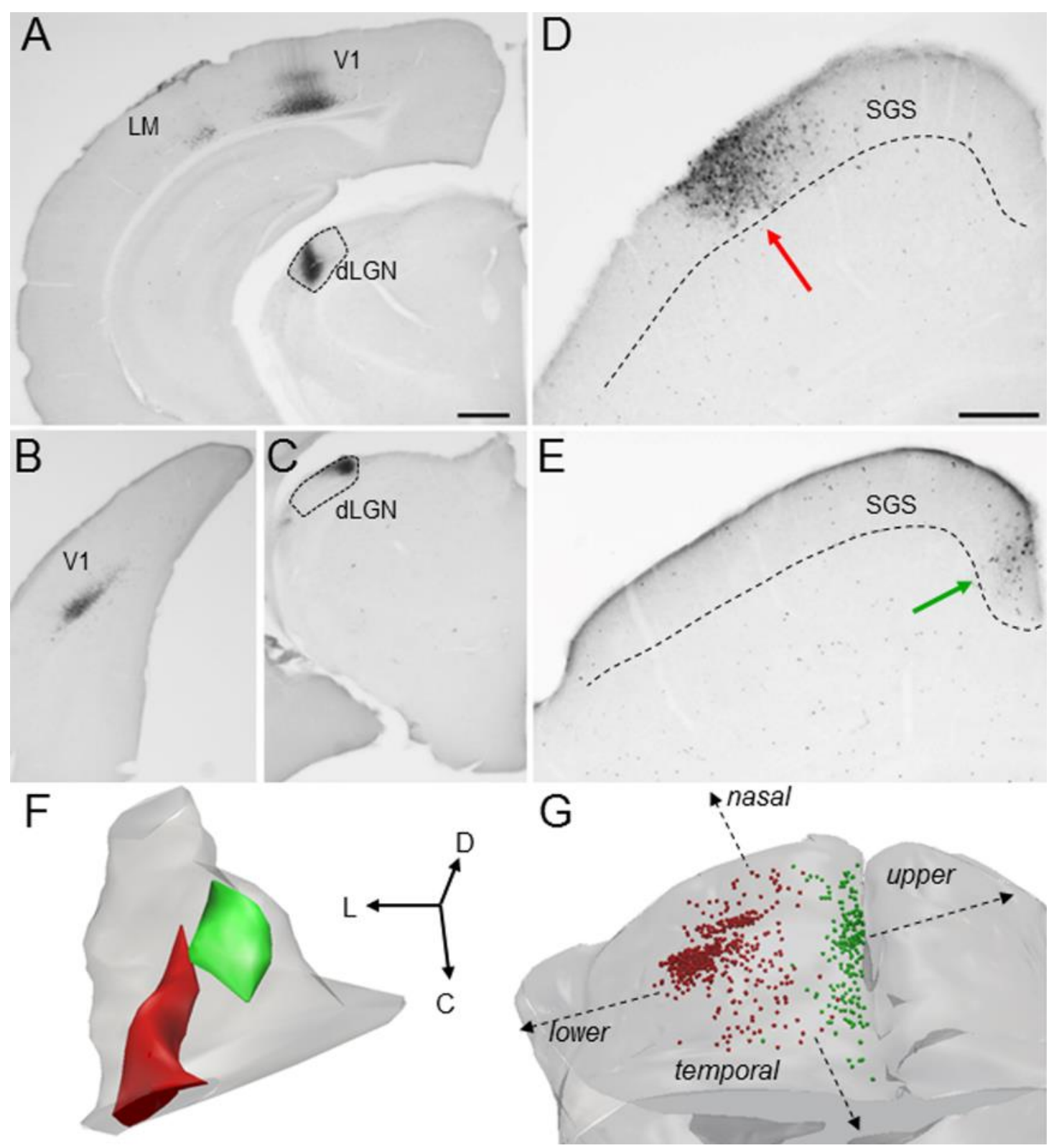


Figure 3. Tectogeniculate projections are primarily nonGABAergic

Injections of CTB-488 were inotophoretically injected into the dLGN of GAD2-cre mice crossed with Ai9 reporter mice. These injections labeled cells in the SGS (A, confocal $6 \mu \mathrm{m}$ optical image) by retrograde transport (green cells) that were largely non-overlapping with the population of GABAergic neurons labeled with tdTomato (red cells). The rectangle in A indicates the region shown in $2 \mu \mathrm{m}$ optical images at higher magnification in B (CTB and tdTomato-labeled cells) and C (tdTomato only). Most CTB-labeled neurons did not contain tdTomato (asterisks), but tdTomato could be detected in $5 \%$ of CTB-labeled cells (e.g. cell indicated by the arrows). Large injections of cre-dependent virus into the SC of GAD2-cre mice (D, pseudocolored green) labeled sparse projections in the dLGN (E, green). Scale bars: $A=50 \mu \mathrm{m}, \mathrm{B}=$ $25 \mu \mathrm{m}$ and also applies to $\mathrm{C}, \mathrm{D}=100 \mu \mathrm{m}$ and also applies to $\mathrm{E}$. 


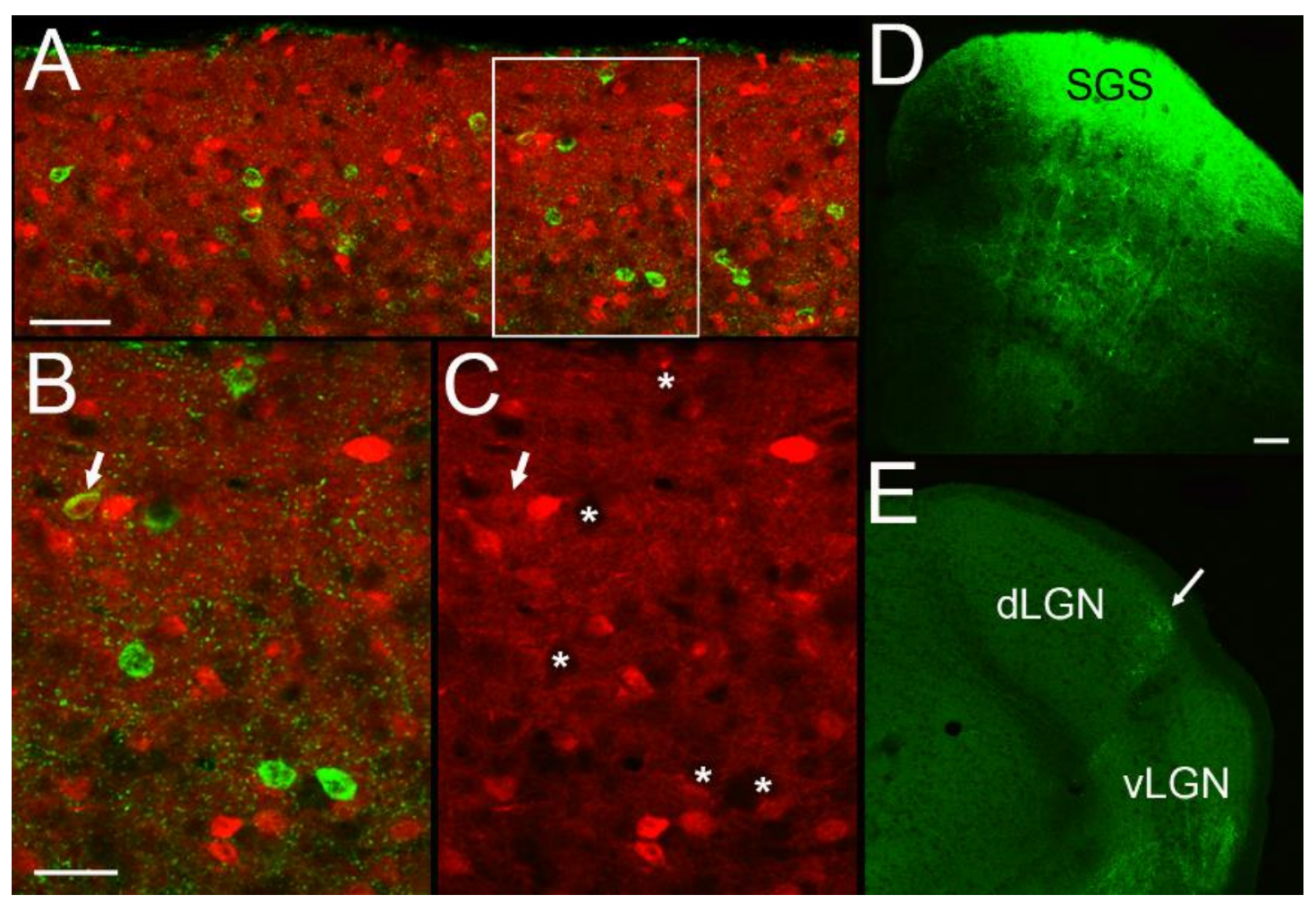




\section{Figure 4. Ultrastructure of tectogeniculate (TG) terminals}

TG terminals (dark reaction product A-F, J, K) are significantly larger than corticogeniculate (CG) terminals (blue, G), and significantly smaller than retinogeniculate terminals (red, G, J, K) identified by their pale mitochondria (*) as RLP profiles. The cumulative distribution of terminals sizes is illustrated in panel $\mathrm{H}$. RLP, TG and CG terminals primarily contact (arrows) nonGABAergic relay cell dendrites (green). GABAergic profiles are identified by a high density of overlying gold particles (purple). The cumulative distribution of postsynaptic dendrite sizes is illustrated in panel I. CG terminals contact dendrites that are significantly smaller than the dendrites contacted by TG and RLP terminals. RLP and TG converge to innervate larger caliber dendrites $(\mathrm{J}, \mathrm{K})$. Scale bar $=1 \mathrm{um}$ and applies to A-G, J, K. 

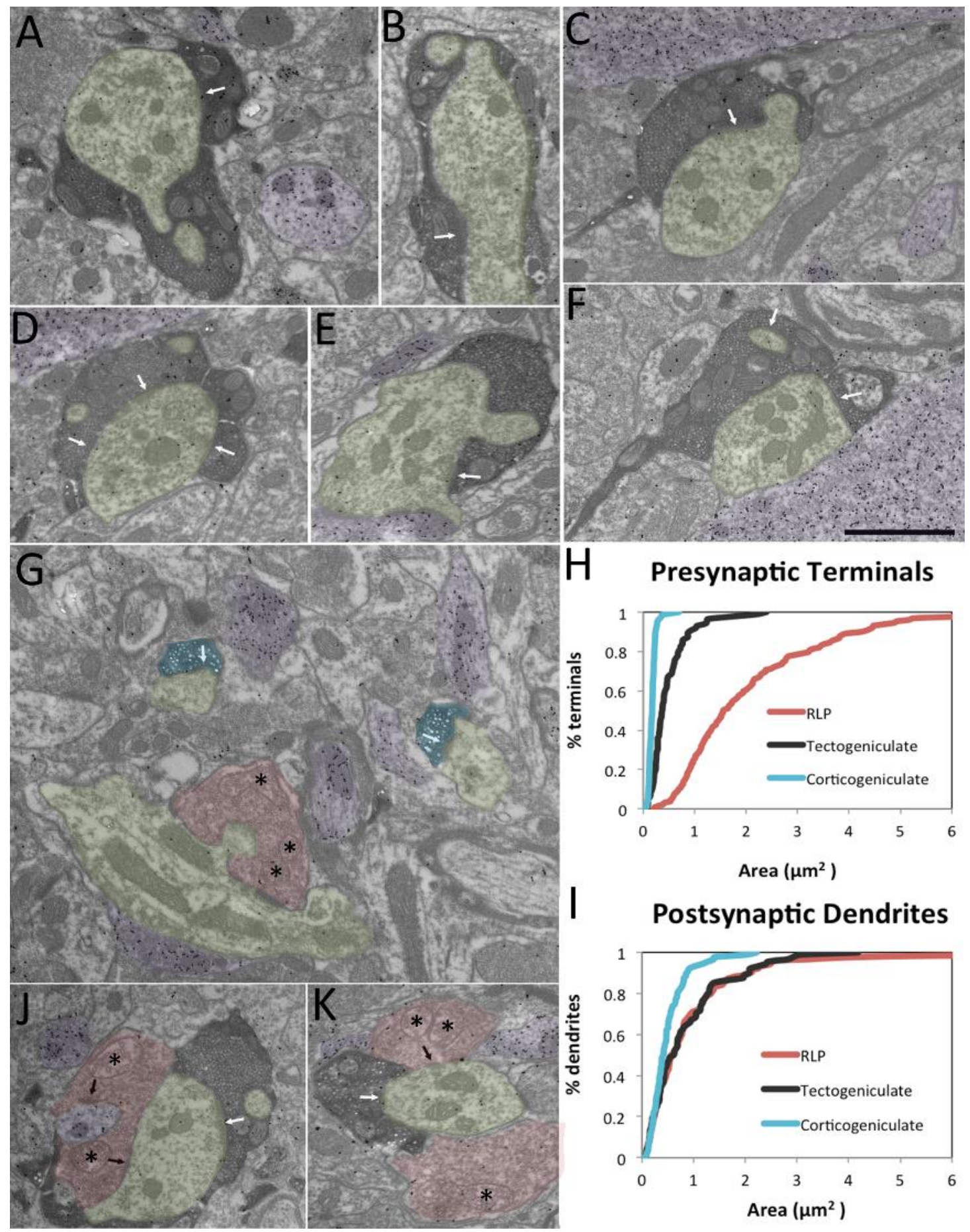


\section{Figure 5. Light-evoked tectogeniculate (TG) responses}

Confocal image of a coronal section of the dorsal lateral shell of the dLGN depicting a W-like biocytin-filled relay neuron (green) and tectogeniculate axons (red) expressing tdTomato following a virus injection in the SC (A). Whole cell currentclamp recording showing large postsynaptic excitatory responses of a dLGN cell evoked by blue light stimulation $(200 \mathrm{~ms}$ pulse) of tectogeniculate terminals expressing ChIEF. Responses recorded at different holding potentials (B). Optically evoked postsynaptic responses of the same cell before and during bicuculline (BIC,

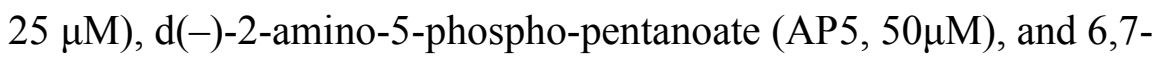
dinitroquinoxaline-2,3-dione (DNQX,10 $\mu \mathrm{M}$ ) application. BIC and AP5 had little to no effect to synaptic response whereas DNQX application completely abolished it (C). A similar effect could be observed when the cell was stimulated by a train of light pulses (20 pulses at $10 \mathrm{~Hz}$; D). 


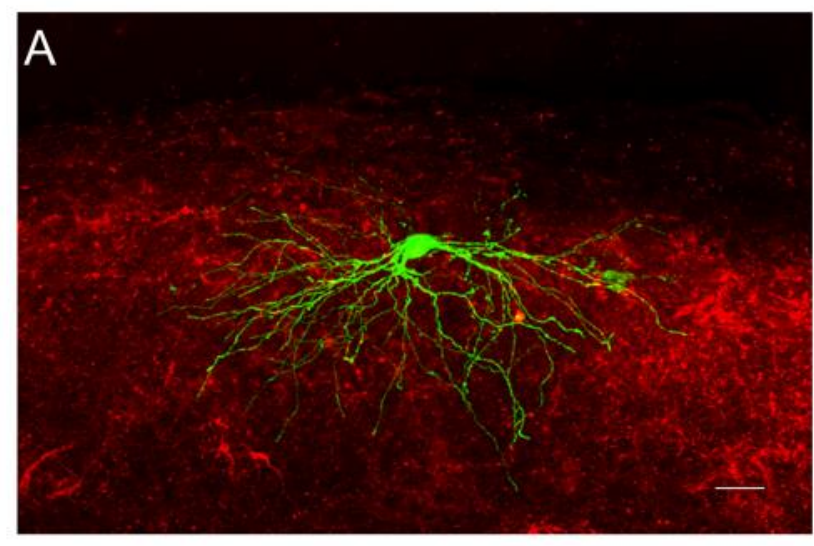

B

C
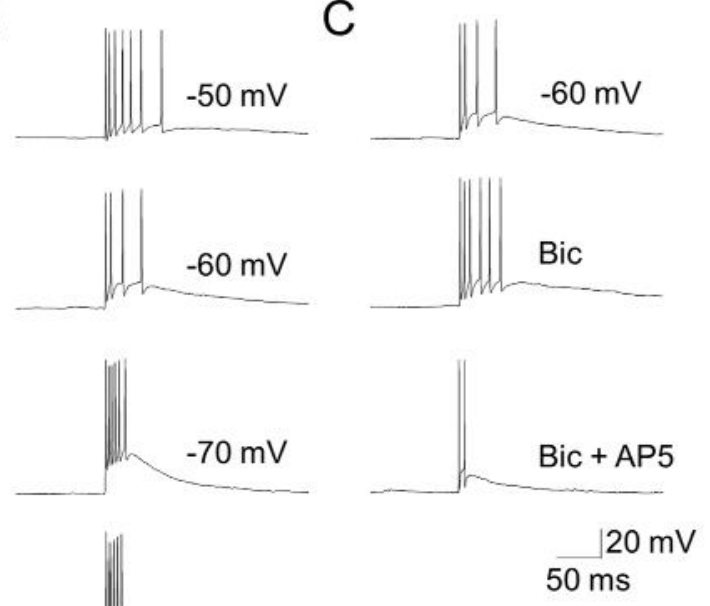

$-80 \mathrm{mV} \quad \mathrm{Bic}+\mathrm{AP} 5+\mathrm{DNQX}$

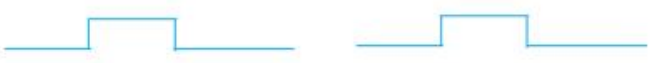

D
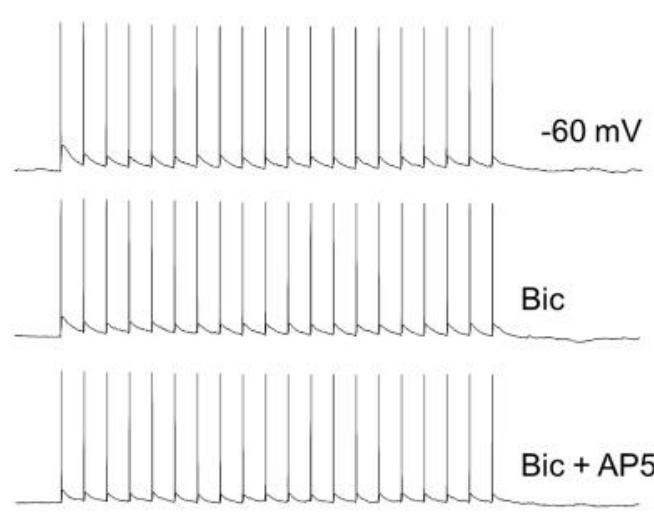

$\mathrm{Bic}+\mathrm{AP} 5+\mathrm{DNQX}$

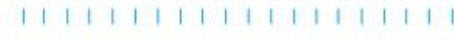

$20 \mathrm{mV}$

$200 \mathrm{~ms}$ 


\section{Figure 6. Synaptic depression of tectogeniculate (TG) responses}

A) Representative voltage-clamp recording of postsynaptic responses in dLGN evoked by paired-pulse light stimulation (100ms interstimulus interval) of ChIEF expressing SC terminals (average of 10 responses; top). Summary plot depicting the paired pulse ratio (EPSC2/EPSC1) for 15 relay cells evoked by the same stimulus conditions (bottom). Gray symbols represent PPR of individual cells and the black symbol represents the mean and S.E.M. PPRs reflect strong synaptic depression. B) Representative recording showing TG synaptic responses evoked by a $20 \mathrm{~Hz}$ train of light (average of 10 responses, top). Summary plot showing the degree of depression of light evoked EPSCs as a function of stimulus number for 12 neurons (bottom). To calculate percent depression the amplitude of the nth response was divided by the first response and multiplied by 100 . 
A

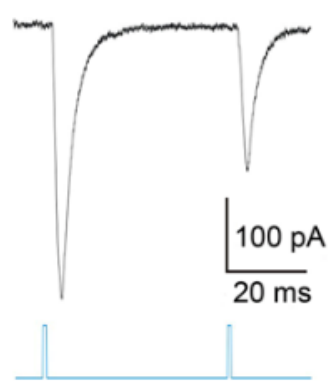

B
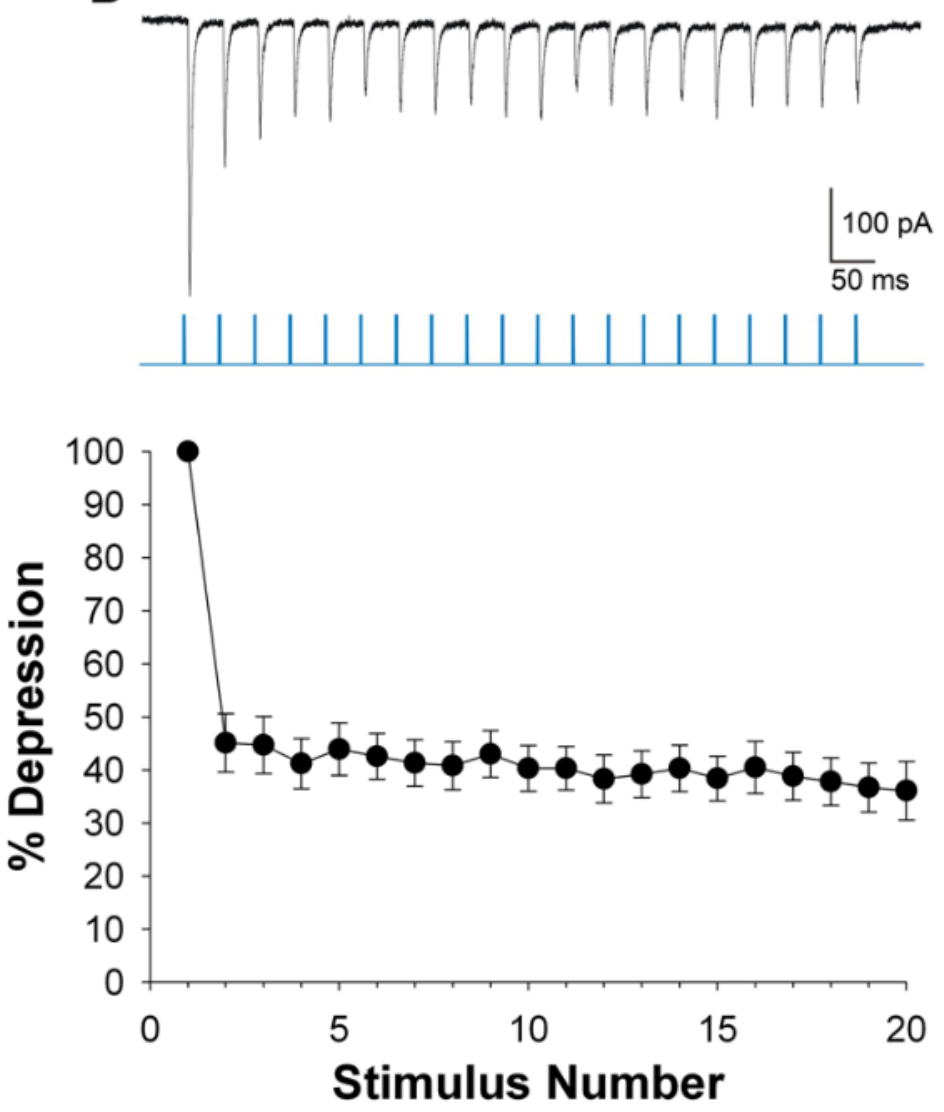


\section{Figure 7. Functional convergence of TG and RG inputs}

A, top: Schematic diagram showing whole-cell recordings from a dLGN relay neuron following electrical stimulation of RG axons in the optic tract, and photostimulation of TG terminals in the dorsolateral shell. Beneath are EPSCs recorded in a single cell evoked by repetitive activation $(20 \mathrm{~Hz})$ of $\mathrm{RG}$ (red traces) and TG (black traces) inputs. Both sets of responses showed synaptic depression. Red and blue ticks indicate corresponding stimulus protocols. A, Inset: Expanded traces of the first 2 RG (red) and TG (black) responses under control conditions. Superimposed gray traces depict responses following bath application of DNQX and CPP; these glutamate receptor antagonists abolished both sets of responses. B, Examples RG (red) and TG (black) EPSCs evoked by paired pulse stimuli (50 and $500 \mathrm{~ms}$ inter-stimulus intervals). C, Summary plot showing the mean and S.E.M. $(\mathrm{n}=6)$ of PPRs for RG (red) and TG (black) EPSCs at 50 and $500 \mathrm{~ms}$ interstimulus intervals. At $50 \mathrm{~ms}$, the TG PPR was smaller indicating greater synaptic depression. Asterisk depicts $\mathrm{p}<0.001$. 
A

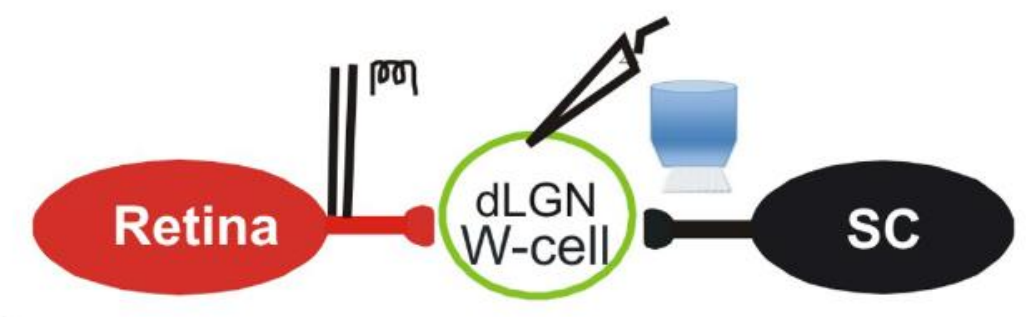

RG

TG

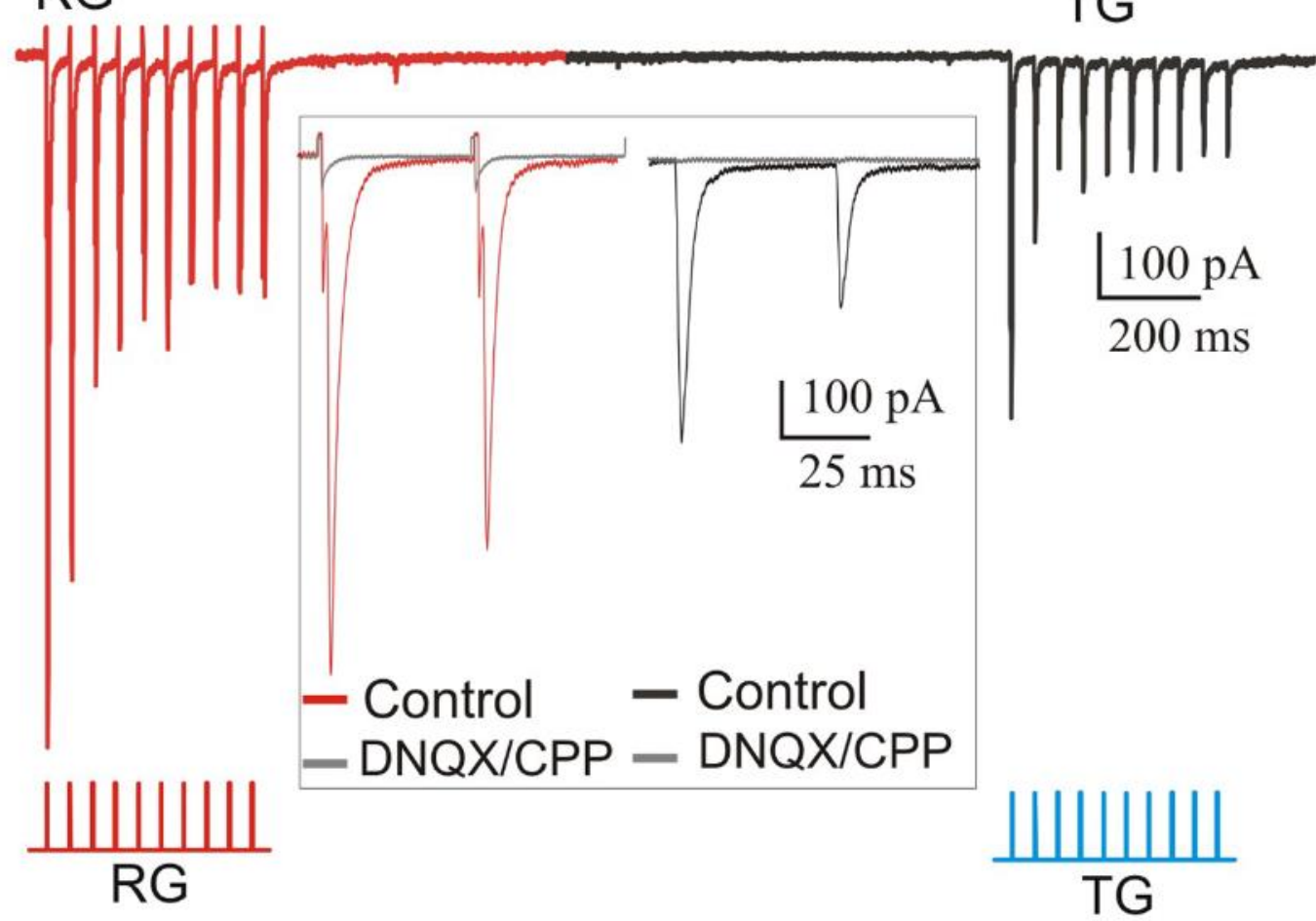

B

$50 \mathrm{~ms}$
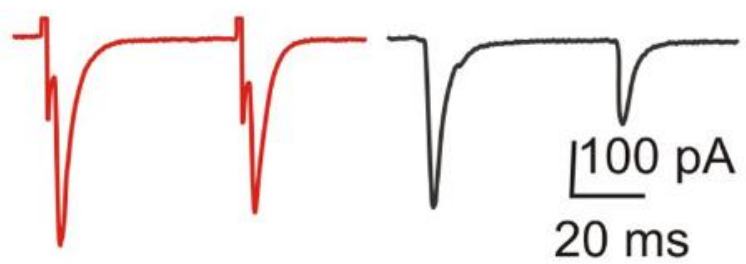

C

$500 \mathrm{~ms}$

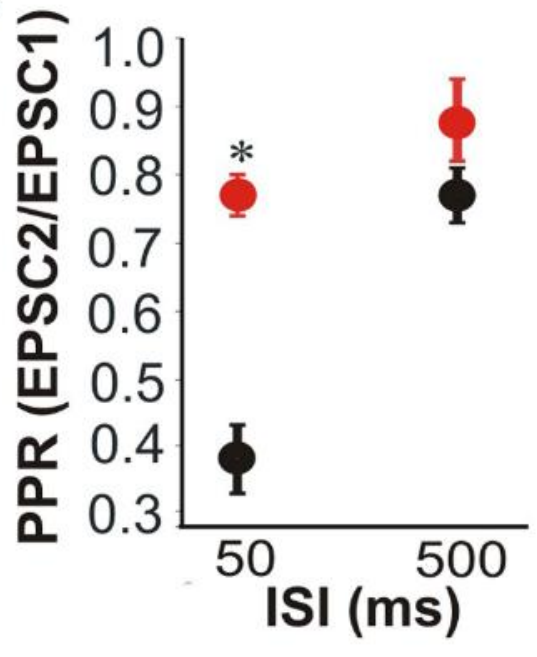




\section{Figure 8. Tectorecipient dLGN neurons project to V1 layer I}

Cholera toxin subunit B conjugated to Alexafuor 488 (CTB-488)-infused filter paper applied to layer I of V1 (A) paired with viral vector injections in the superior colliculus (SC, B) resulted in the retrograde labeling of dorsal lateral geniculate nucleus (dLGN) cells (green, C) in regions of the dLGN innervated by tectal terminals (red, C). CTBlabeled cells (green, D) filled with biocytin (red, D) responded to photoactivation of surrounding tectogeniculate terminals with large amplitude EPSCs that exhibited frequency-dependent depression (E). LPN, lateral posterior nucleus, OT, optic tract, SC, superior colliculus. Scale bars: A-C $=100 \mu \mathrm{m}, \mathrm{D}=20 \mu \mathrm{m}$. 


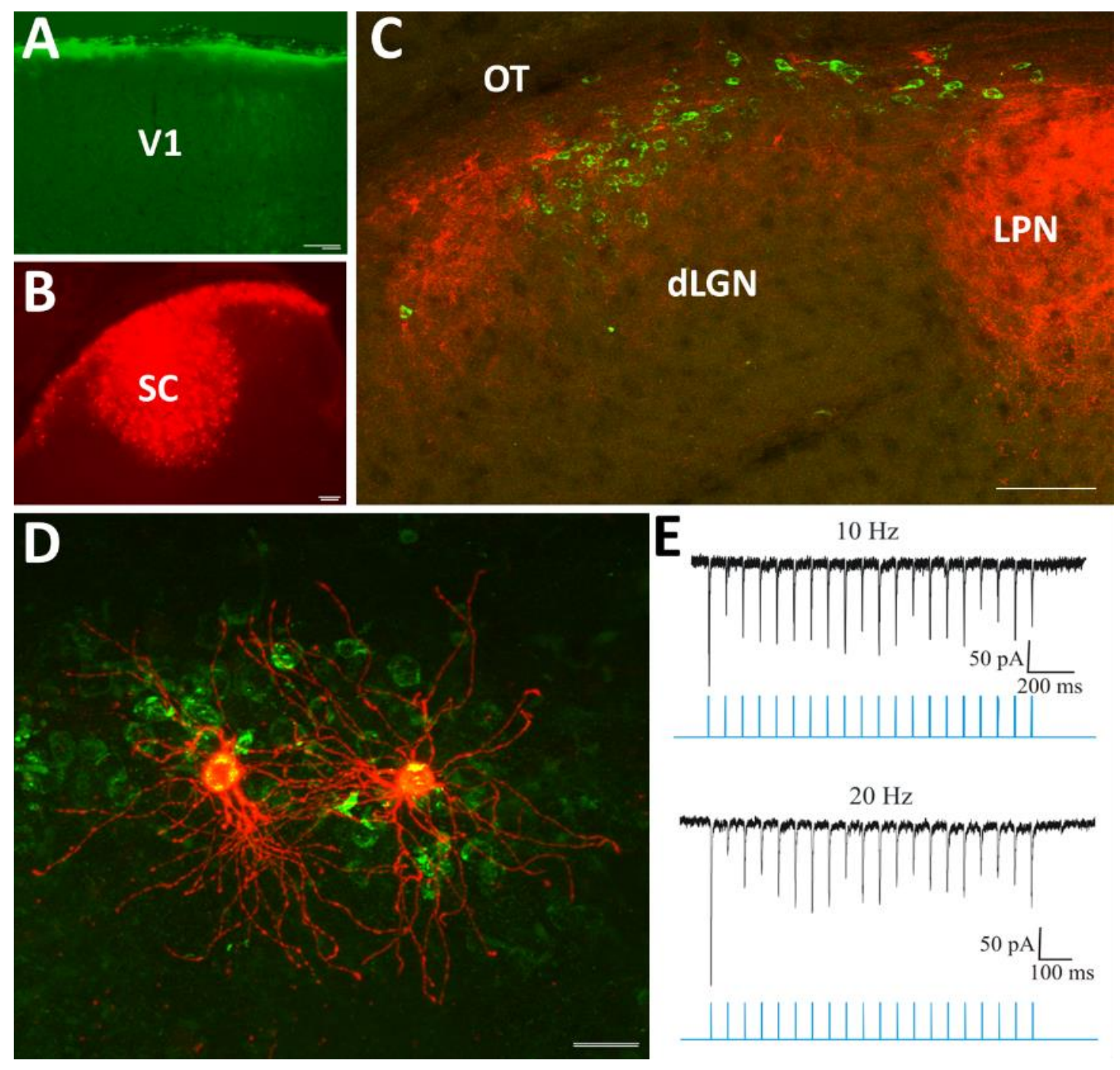




\section{Figure 9. Synaptic targets of dLGN projections to V1 layer I}

Injections of biotinylated dextran amine (BDA) in the dLGN (A, inset) labeled terminals that were distributed primarily in layer IV of V1 (A), but also innervated layer I of V1 (A, arrows, panel B). Electron microscopic analysis of BDA-labeled geniculocortical terminals in layer I of V1 indicated that the majority $(98 \%)$ of these terminals contact ( $\mathrm{C}$, arrows) nonGABAergic dendrites (C, pink). LPN, lateral posterior nucleus, OT, optic tract, SC, superior colliculus. Scale bars: $A=100 \mu \mathrm{m}, \mathrm{A}$ inset $=100$ $\mu \mathrm{m}, \mathrm{B}=10 \mu \mathrm{m}, \mathrm{C}=0.5 \mu \mathrm{m}$ 

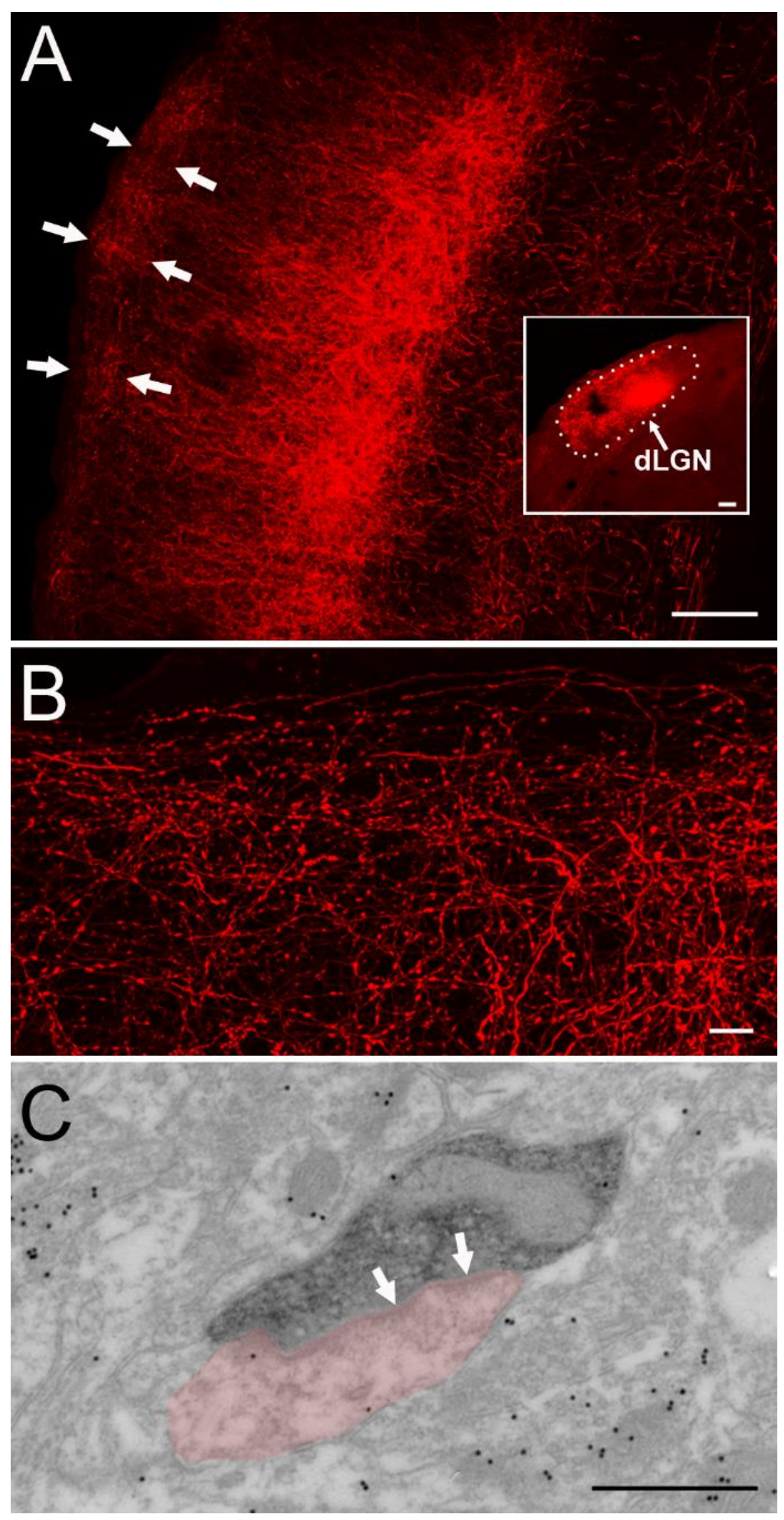


\section{Figure 10. Distinct functional circuits in the dLGN core and shell}

The summary diagram depicts the circuits of the dLGN shell revealed in the current study on the left side, and the circuits of the dLGN core revealed in previous studies on the right side.

Core: The core contains cells that display "X-like" and "Y-like" morphology (Krahe et al., 2011). These neurons receive input from V1 (gray, RS) on their distal dendrites and input from non-direction-selective (non-DS) retinal ganglion cells (green, RLP) on their proximal dendrites, which drive center-surround receptive field properties (Bickford et al., 2010; Huberman et al., 2008; Kay et al., 2011; Kim et al., 2010; Piscopo et al., 2013). Core cells project to layer IV of VI (Cruz-Martín et al., 2014).

Shell: The shell contains cells that display "W-like" morphology. Like core neurons, shell neurons receive input from the cortex (gray, RS) on their more distal dendrites, but their proximal dendrites are innervated by convergent input from direction-selective (DS) retinal ganglion cells (blue, RLP; Cruz-Martín et al., 2014), as well as the superior colliculus (red, RM). Presumably, the integration of DS retinal and SC inputs underlies emergent direction-selective properties of dorsolateral shell neurons (Marshel et al., 2012; Piscopo et al., 2013; Scholl et al., 2013; Zhao et al., 2013). Shell neurons project to layer I (current results as well as those of Cruz-Martin et al., 2014), where they contact the dendrites of nonGABAergic pyramidal cells. 


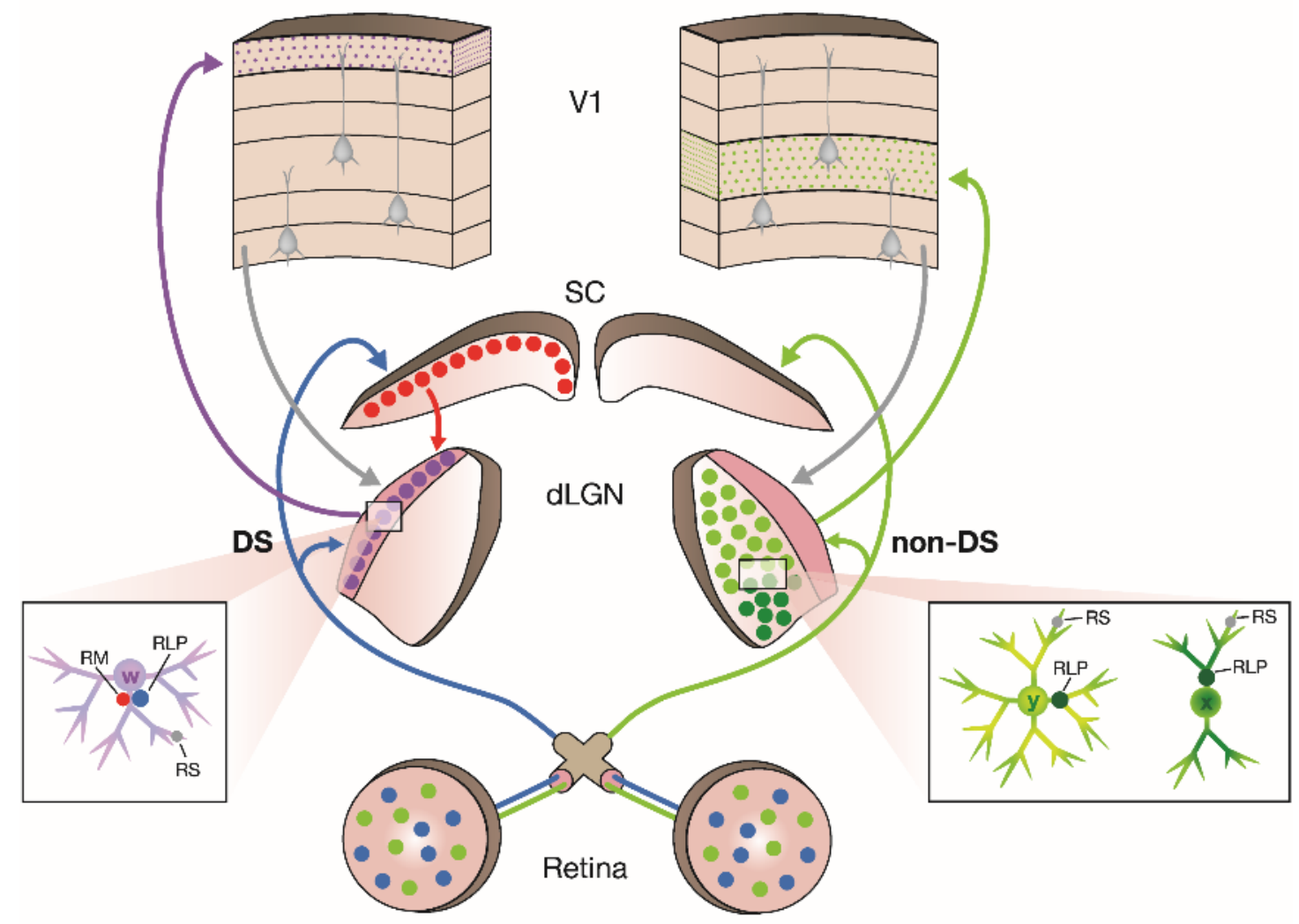




\section{CHAPTER III}

\section{THE MOUSE PULVINAR NUCLEUS: ORGANIZATION OF THE}

\section{TECTORECIPIENT ZONES ${ }^{2}$}

\section{Overview}

Comparative studies have greatly contributed to our understanding of the organization and function of visual pathways of the brain, including that of humans. This comparative approach is a particularly useful tactic for studying the pulvinar nucleus, an enigmatic structure which comprises the largest territory of the human thalamus. This review focuses on the regions of the mouse pulvinar that receive input from the superior colliculus, and highlights similarities of the tectorecipient pulvinar identified across species. Open questions are discussed, as well as the potential contributions of the mouse model for endeavors to elucidate the function of the pulvinar nucleus.

The pulvinar nucleus is considered one of the most enigmatic thalamic regions. Factors that contribute to its mystery are the vast array of anatomical connections that involve the pulvinar nucleus, its reduced activity in anesthetized or restrained animals, and the resulting difficulties in determining the circuits and stimuli that contribute to its receptive field properties. Additionally, although the pulvinar is commonly considered a single thalamic nucleus, it contains a number of distinct subregions which may be

2. Zhou N, Maire PS, Masterson SP, Bickford ME. Vis Neurosci. Published online: 27 June 2017. DOI. https://doi.org/10.1017/S0952523817000050. 
differentially involved in the various functions ascribed to the pulvinar (e.g. visual attention, decision making, motor planning, perceptual suppression, synchronization of cortical activity, detection of faces or fearful stimuli; Dominguez-Vargas et al., 2017; Grimaldi et al., 2016; Van Le et al., 2014; Le et al., 2014, 2016; McFadyen et al., 2017; Soares et al., 2017; Wilke et al., 2009, 2010, 2013; Zhou et al., 2016). In order to understand how the pulvinar contributes to these various tasks, the synaptic circuits within each subregion must first be defined.

This review focuses on circuits of the mouse lateral posterior nucleus (LPN), a region considered to be the homologue of the primate pulvinar nucleus (Harting et al., 1972). As schematically illustrated in Figure 11, this homology is based to a large extent on commonalities in the projections of the superficial (visual) layers of the superior colliculus (SC), or optic tectum, to the primate pulvinar nucleus, rodent/carnivore LPN, and avian nucleus rotundus (Harting et al., 1973b; Robson and Hall, 1977; Berson and Graybiel, 1978; Mooney et al., 1984; Takahashi, 1985; Abramson and Chalupa, 1988; Luppino et al., 1988; Hutsler and Chalupa, 1991; Villeneuve and Casanova, 2003; Kelly et al., 2003; Chomsung et al., 2008; Masterson et al., 2009, 2010; Baldwin et al., 2011; Wei et al., 2011b; Fredes et al., 2012; Baldwin et al., 2013). Because of these similarities, we will refer to this region of the mouse thalamus as the pulvinar nucleus. We hope that this nomenclature will assist in comparative studies that may contribute to our understanding of the organization and function of the pulvinar nucleus across species, including that of humans. In order to most explicitly relate the organization of the mouse pulvinar to that of other species, this review emphasizes the regions that receive 
input from the SC. Although the size of the tectorecipient zones relative to the entire extent of the pulvinar nucleus varies across species, there are a number of similarities in the organization of these zones as discussed below.

\section{Tectopulvinar cells}

The SC projections to the pulvinar nucleus originate from a unique class of cells, termed widefield vertical (WFV) cells (Figure 12). WFV cells have been identified in a variety of species (chicken, pigeon, mouse, rat, ground squirrel, gray squirrel, tree shrew; (Mooney et al., 1988; Karten et al., 1997b; Luksch et al., 1998, 2001; Major et al., 2000; Marín et al., 2003; May, 2006; Chomsung et al., 2008; Endo et al., 2008; Isa and Hall, 2009; Kaneda et al., 2011; Fredes et al., 2012; Gale and Murphy, 2014); in each case these cells display very large dendritic fields that cover significant regions of the SC or optic tectum. Based on the configuration of their dendritic arbors, and interaction with retinotectal inputs in vitro (Luksch et al., 2001; Endo et al., 2008), WFV cells have been referred to as motion detectors (Major et al., 2000). This concept has been corroborated in vivo in the mouse, where it has been demonstrated that WFV cells respond best to a small visual stimulus moving in any direction within a large visual field (Gale and Murphy, 2014, 2016).

In the ground squirrel, two types of WFV cells have been identified. Type I WFV cells extend their dendrites to the most superficial extent of the SC (within the most dorsal regions of the stratum griseum superficiale, or SGS), while type II WFV cell dendrites end in the middle of the SGS (Major et al., 2000). These two cell types have been found to project to different regions of the pulvinar nucleus (Fredes et al., 
2012; described in more detail below). Similar to type I and type II WFV cells, the dendrites of type I and type II tectorotundal cells end in different lamina of the chick optic tectum (Luksch et al., 1998), and each type responds differentially to electrical stimulation of retinal input (Luksch et al., 2001).

In the mouse, WFV cells have not been subdivided. However, the availability of transgenic mouse lines (e.g. Byun et al., 2016; Gale and Murphy, 2014, 2016) may help to facilitate the categorization of these cells. If subclasses of WFV cells exist in the mouse, those that extend dendrites most superficially within the SC (Figure 12C) could potentially be innervated by populations of retinal axons that are restricted to the most superficial regions of the SGS (e.g. those that originate from directionselective ganglion cells; Rivlin-Etzion et al., 2011). Future studies in mice may take advantage of ganglion cell-specific transgenic lines to determine whether WFV cells are innervated by single ganglion cell subtypes (to form dedicated parallel channels of information flow to the pulvinar) or whether they receive convergent input from multiple classes of ganglion cells.

\section{Tectopulvinar projection patterns}

The projections of WFV cells target specific subregions of the pulvinar. In the mouse, the caudal medial pulvinar $(\mathrm{Pcm})$ receives bilateral input from WFV cells and the lateral pulvinar $(\mathrm{Pl})$ receives input from ipsilateral WFV cells (Figure 13). Similar projection patterns have previously been identified in the rat (Takahashi, 1985), and these two subdivisions can be distinguished with a variety of immunocytochemical markers (Nakamura et al., 2015). In the mouse, the Pcm contains a dense population 
of terminals that contain substance P (Figure 14). Similarly, the primate posterior (PIp) and central medial (PIcm) subdivisions of the inferior pulvinar (Figure 11) also contain a dense population of terminals that stain for substance P (Stepniewska et al., 2000). The mouse Pcm can also be defined based on cells that contain both the calcium-binding protein calretinin and express the substance P receptor neurokinin 1 (NK1, Figure 13); in contrast, the Pl does not stain with antibodies against substance P, NK1, or calretinin (Figures 13 and 14).

The organization of tectorecipient zones in the mouse pulvinar is very similar to that identified in the ground squirrel, where the caudal pulvinar receives bilateral, nontopographic SC projections that originate from type I WFV cells, while the rostral pulvinar receives topographic, ipsilateral SC projections that originate from type II WFV cells (Fredes et al., 2012). As illustrated in Figure 11, two types of tectopulvinar projections, nontopographic or "diffuse" projections and topographic "specific" projections, have also been identified in gray squirrels (Baldwin et al., 2011), tree shrews (Chomsung et al., 2008; Luppino et al., 1988), and galagos (Baldwin et al., 2013). In the tree shrew, the nontopographic tectal projections are highly convergent. These tectopulvinar terminals form dense clusters that surround and synapse on single pulvinar dendrites. In contrast, the topographic projections are less convergent and form smaller, more discrete, synaptic clusters (Chomsung et al., 2008; Wei et al., 2011b). These two tectopulvinar innervation patterns have been revealed across species using antibodies against the type 2 vesicular glutamate transporter (vGLUT2, contained in tectopulvinar terminals; Wei et al., 2011b); vGLUT2 staining is very 
dense in regions of the pulvinar that receive convergent tectal input, and lighter in regions that receive topographic tectal projections (Chomsung et al., 2008; Baldwin et al., 2011, 2013). Multiple tectopulvinar pathways that originate from separate SC cell types have also been identified in the cat (Abramson and Chalupa, 1988; Kelly et al., 2003), and in the pigeon, a unique interdigitated pattern of tectorotundal projections originate from separate optic tectum cell types (Marín et al., 2003).

The precise organization of tectopulvinar projections has not been studied in mice. Tracing the axonal projections of single WFV cells would facilitate our understanding of the organization and potential topography of this pathway. Monosynaptic circuit tracing (Wickersham et al., 2007) in transgenic mice (e.g. calretinin-cre mice), could also help to determine whether subclasses of WFV cells target distinct pulvinar subdivisions. In many species, the pulvinar has been subdivided using histochemical staining for the enzyme acetylcholinesterase and/or immunohistochemical staining for the neuromodulator substance P (Graybiel and Berson, 1980; Abramson and Chalupa, 1988; Luppino et al., 1988; Hutsler and Chalupa, 1991; Stepniewska et al., 1999; Kelly et al., 2003; Chomsung et al., 2008; Baldwin et al., 2011, 2013; Fredes et al., 2012). Where examined, these two stains overlap to a great extent, perhaps due to involvement of acetylcholinesterase in the hydrolysis of substance P (Goebel and Pourcho, 1992). Studies in the cat and rat suggest that the expression of substance $\mathrm{P}$ in tectopulvinar pathways is developmentally regulated, and influenced by visual input (Miguel-Hidalgo et al., 1990, 1991; Behan et al., 1993). The mouse is an ideal model to further define the role 
of substance $\mathrm{P}$ in tectopulvinar pathways by using transgenic lines, optogenetics, and/or designer receptors exclusively activated by designer drugs (DREADD) to manipulate substance $\mathrm{P}$ pathways and characterize any resulting behavioral effects.

\section{Synaptic properties of tectopulvinar terminals}

Tectopulvinar terminals have consistently been found to form clusters of relatively large terminals that surround and synapse on the proximal dendrites of pulvinar neurons (Partlow et al., 1977; Robson and Hall, 1977; Crain and Hall, 1980a; Kelly et al., 2003; Chomsung et al., 2008; Masterson et al., 2009; Wei et al., 2011b; Bickford, 2015); tectopulvinar terminals in the mouse exhibit similar characteristics (Figure 15B). In vitro slice studies in the rat and tree shrew have demonstrated that multiple tectopulvinar axons can converge on single cells (Masterson et al., 2010; Wei et al., 2011b), presumably contributing to the large receptive fields of pulvinar neurons (Chalupa et al., 1983; Mooney et al., 1984; Chalupa and Abramson, 1988; Casanova et al., 2001; Dumbrava et al., 2001; Berman and Wurtz, 2011a; Roth et al., 2016).

Tectopulvinar terminals release glutamate to activate ionotropic glutamate receptors on postsynaptic neurons (Masterson et al., 2010; Wei et al., 2011c). Stimulation of tectopulvinar terminals at frequencies of up to $20 \mathrm{~Hz}$ elicits postsynaptic responses that maintain relatively stable amplitudes (unlike the frequency-dependent amplitude changes demonstrated in other thalamic pathways; for review see Bickford, 2016). This frequency-independence may be due to the synaptic arrangements of these terminals and/or the presynaptic proteins contained within them (synapsin I and synapsin II; Wei et al., 2011b). Another unique feature of 
tectopulvinar terminals is that stimulation at $100 \mathrm{~Hz}$ can elicit their release of substance $\mathrm{P}$ which, through activation of neurokinin 1 receptors, can boost tectopulvinar responses (Masterson et al., 2010).

Again, the mouse is an ideal model to study further details of the synaptic properties of tectopulvinar terminals. These terminals can be specifically activated using optogenetic techniques (Maire et al., 2015) and transgenic lines (e.g. mice that lack synapsins; Kielland et al., 2006; Song and Augustine, 2015) can potentially be used to determine the mechanisms that underlie their unique frequency-independence. Studies in mice may also reveal whether substance $\mathrm{P}$ is contained in all tectopulvinar projections, or confined to those originating from specific WFV subclasses. Our previous in vitro studies in the rat suggested that all tectopulvinar projections contain substance P (Masterson et al., 2010). However, our investigation was limited to the caudal most regions of the pulvinar (likely corresponding to the mouse Pcm; Figure 14).

\section{Retinal innervation and plasticity of pulvinar pathways}

Tecto-pulvinar pathways have often been cited as the substrate mediating "blindsight": the ability, in the absence of visual perception, to navigate using visual cues and respond to negative or fearful facial expressions (Leopold, 2012; Schmid and Maier, 2015). However, it has recently been demonstrated that during development, the pulvinar transiently receives substantial direct input from the retina, which diminishes to sparser levels in adults. This pathway shows considerable plasticity: in situations where $\mathrm{V} 1$ is lost at an early age, this retinopulvinar pathway 
does not regress, and may account for the preservation of vision when lesions to $\mathrm{V} 1$ occur during infancy (Warner et al., 2012; Kaas, 2015; Bridge et al., 2016).

In the mouse, it has been demonstrated that at least some of the retinopulvinar projections arise from intrinsically photosensitive (melanopsin-containing) ganglion cells, and a portion of pulvinar neurons are functionally influenced by melanopsinderived signals (Allen et al., 2016). A melanopsin-dependent light aversion response in neonatal mice activates pulvinar cells, as well as cells in the amygdala (which as discussed below, receives input from the pulvinar; Delwig et al., 2012). Perhaps, as in primates, direct retinopulvinar projections in the mouse are also more robust during development and function to initiate basic movements in response to light. However, it is still unknown how direct retinopulvinar versus indirect retino-tecto-pulvinar pathways contribute to melanopsin-dependent pulvinar responses, and motor behaviors.

Lesion studies in the hamster demonstrated that terminals originating from the retina, SC and cortex all compete for territory in the developing pulvinar nucleus; retinopulvinar terminations expand after SC lesions and/or combined SC and cortex lesions (Crain and Hall, 1980, 1981). Further investigations in mice may help to define mechanisms underlying the developmental competition between retinopulvinar, tectopulvinar and corticopulvinar projections, and how this might correlate with transitions from the simple light-aversive movements of neonates to the more complex visually-guided escape, freezing or prey capture behaviors of adult mice (Yilmaz and Meister, 2013; De Franceschi et al., 2016; Hoy et al., 2016). 


\section{The striate-recipient zones of the pulvinar}

Across mammalian species, the pulvinar also contains zones that are innervated by the striate cortex (cat; Berson and Graybiel, 1983; Guillery et al., 2001; Huppé-Gourgues et al., 2006; rat; Li et al., 2003c; macaque; Ogren and Hendrickson, 1979). In rodents, terminals that originate from $\mathrm{V} 1$ innervate the $\mathrm{Pl}$, as well as more rostral thalamic regions (the rostral medial pulvinar, Prm, and lateral dorsal nucleus, LD; Bourassa and Deschenes 1995; Rubio-Garrido et al 2009). These more rostral regions are well segregated from the tectorecipient zones. However, the mouse P1 shows considerable overlap in the distribution of terminals originating from the SC and V1 (Figure 16L, 17B). The striate- and tectorecipient zones of the pulvinar are also well segregated in other species, but may contain some zones of overlap (e.g. the cat LP1-2; Abramson and Chalupa, 1988; Chalupa and Abramson, 1989; HuppéGourgues et al., 2006; Kelly et al., 2003).

The striate-recipient zones of the mouse pulvinar form reciprocal connections with V1, with pulvinocortical projections to V1 ending primarily in layers I and V (Figure 18B; Herkenham, 1980; Roth et al., 2016). Retrograde tracing studies in the mouse indicate that the pulvinocortical projections to V1 are organized in a roughly topographic manner, but this organization is clearly different from the precise topography of connections between V1 and the dorsal lateral geniculate nucleus (dLGN; Roth et al., 2016). In addition, tracing of single axons in the rat indicates that individual pulvinar cells that project to $\mathrm{V} 1$ also send projections to various areas of the extrastriate cortex, as well as the striatum (Nakamura et al., 2015). 
$\mathrm{V} 1$ projections to the pulvinar have been shown to arise from cells in layer $\mathrm{V}$, as well as cells in lower layer VI (cat; Abramson and Chalupa, 1985; rat; Bourassa and Deschênes, 1995; galago; Conley and Raczkowski, 1990; macaque; Lund et al., 1975; mouse; Roth et al., 2016). The terminals that arise from layer V cells are significantly larger than corticogeniculate terminals or tectopulvinar terminals (rat; Bourassa and Deschênes, 1995; tree shrew; Chomsung et al., 2008; Day-Brown et al., 2017; cat; Guillery et al., 2001; Kelly et al., 2003; Huppé-Gourgues et al., 2006; rat; Li et al., 2003c; Masterson et al., 2009), and similar large V1 corticopulvinar terminals are found in the mouse (Figure 15A, 17A).

Extrastriate connections of the mouse pulvinar nucleus

Visual areas of the mouse cortex have been defined on the basis of corticocortical connections with V1 (Wang and Burkhalter, 2007). In this way nine distinct visual areas that surround V1 have been identified: posterior $(\mathrm{P})$, postrhinal (POR), lateromedial (LM), laterointermediate (LI), anterolateral (AL), rostrolateral (RL), anterior (A) anteromedial (AM), and posterormedial (PM). All of these extrastriate visual areas are reciprocally connected to the mouse pulvinar nucleus (Tohmi et al., 2014), and also innervate the superior colliculus (Wang and Burkhalter, 2013). The tectorecipient zones of the pulvinar are primarily connected with the lateral extrastriate cortex (LES, Figure 16C, G; primarily areas P, POR, LM and LI). These connections are roughly topographic, with the Pcm forming reciprocal connections primarily with more ventral regions ( $\mathrm{P}$ and $\mathrm{POR}$ ) and the $\mathrm{P} 1$ primarily forming connections with more dorsal regions adjacent to V1 (LM and LI; Figure 18, 
9; Tohmi et al., 2014). However, given the widespread projections of single pulvinocortical axons identified in the rat (Nakamura et al., 2015), the exact organizational scheme of pulvinocortical projections remains an open question.

Within the extrastriate cortical areas connected with the tectorecipient pulvinar, pulvinocortical terminals are concentrated in layer IV, and corticopulvinar cells are concentrated in layer VI (Figure 18D; Abramson and Chalupa, 1985; Chomsung et al., 2010; Herkenham, 1980; Masterson et al., 2009; Nakamura et al., 2015; Roth et al., 2016). Cortical terminals that innervate the tectorecipient zones of the pulvinar nucleus primarily form smaller terminals that innervate smaller, distal dendrites (Figure 15C; Chomsung et al., 2010; Masterson et al., 2009; Robson and Hall, 1977). Electrical stimulation of corticopulvinar terminals in tectorecipient zones of the rat initially elicits small amplitude glutamatergic excitatory postsynaptic potentials (EPSPs), but repetitive stimulation rapidly increases EPSP amplitudes in a frequency-dependent manner (Masterson et al., 2010). This contrasts with electrical activation of corticopulvinar terminals in more rostral regions of the rat pulvinar nucleus, where a second type of large amplitude EPSP can also be elicited, which exhibits a frequencydependent decrease in amplitude (Li et al., 2003b). These two types of EPSPs, which presumably result from the activation of terminals that originate from layer $\mathrm{V}$ or layer VI corticopulvinar cells, also differ in the degree of convergence onto single pulvinar neurons. Electrical stimulation of layer VI corticopulvinar axons with increasing current levels results in a graded increase in the amplitude of postsynaptic responses, demonstrating that many terminals converge on postsynaptic neurons. In contrast, 
electrical stimulation of layer $\mathrm{V}$ corticopulvinar axons with increasing current levels results in "all or none" changes in the amplitude of postsynaptic responses, demonstrating that each postsynaptic neuron receives input from only a few of these axons (Li et al., 2003b; Masterson et al., 2010).

The function of layer V versus layer VI corticopulvinar projections is still unclear. It has been proposed that layer $\mathrm{V}$ corticopulvinar projections function to transfer signals from one cortical area to another (Guillery and Sherman, 2002). It has also been suggested that layer $\mathrm{V}$ corticothalamic projections could function to detect the relative timing of sensory events and ongoing cortical activity (Groh et al., 2008). Experiments in mice could be designed to specifically manipulate the activity of layer V versus layer VI corticopulvinar projections to determine the effects on pulvinar activity, cortical activity and/or behavior. Such experiments would be particularly important for testing the hypothesis that layer $\mathrm{V}$ corticopulvinar projections are the primary determinant (“drivers”) of pulvinar neuron receptive field properties (Sherman and Guillery, 1998).

\section{Pulvinar projections to the striatum and amygdala}

The tectorecipient zones of the pulvinar also project to the striatum and lateral amygdala (Takahashi, 1985; Harting et al., 2001; McHaffie et al., 2005; Day-Brown et al., 2010; Nakamura et al., 2015; Roth et al., 2016), suggesting pulvinar involvement in the visual guidance of movement. Recently, activation of the mouse SC-pulvinaramygdala pathway has been shown to elicit freezing responses, while inactivation of this pathway inhibits the innate freezing response to overhead looming stimuli (Wei et 
al., 2015). Similar pathways have been implicated in visually-triggered fear responses across species (Carr, 2015).

In the tree shew, pulvinar-amygdala cells are concentrated in the regions of the pulvinar that receive the non-topographic projections from the SC (Pd, Figure 1, DayBrown et al., 2010). Likewise, mouse pulvinar-amygdala cells appear to be concentrated in the Pcm (Wei et al., 2015). In the rat, SC contacts on pulvinaramygdala cells have been identified (Linke et al., 1999), and cells in regions corresponding to the Pcm branch to innervate the ventral temporal cortex and amygdala (Doron and Ledoux, 2000), or caudal striatum (Nakamura et al., 2015). Thus, the bilateral SC-pulvinar-amygdala pathway (Figure 19A) may primarily function to activate freezing or escape responses. Mice could be used for future studies to determine whether the unilateral SC-pulvinar-striatum projections (Figure 19B) trigger distinct motor responses, such as prey capture (Hoy et al., 2016). Cell types within the pulvinar nucleus

Our understanding of the organization of the dLGN was greatly advanced by the identification of morphological cells types that correlate with functional cell classes (e.g. Friedlander et al., 1981); identification of structure/function correlations for pulvinar neurons is expected to similarly advance our understanding of this nucleus. The pulvinar contains projection cells (Figure 17C; Nakamura et al., 2015), GABAergic interneurons (Figure 17D; Carden and Bickford, 2002; Chomsung et al., 2008; Li et al., 2003c), and a dense population of glial cells (glial to neuron ratio of approximately 3:1 in the tree shrew pulvinar; Wei et al., 2011a). In the rat, the axons 
of individual projection cells have been shown to innervate multiple cortical areas, multiple cortical lamina, as well as the striatum and amygdala (Nakamura et al., 2015). Evidence in the cat and primate also suggests that pulvinar axons innervate widespread cortical areas (Kaufman et al., 1984; Baleydier and Mauguière, 1987; Rockland, 2002). Therefore, the subdivision of pulvinar neurons based on projection targets is not straightforward.

In addition, the dendrites of pulvinar neurons are not restricted to specific input zones (Figure 17C, D; Ogren and Hendrickson, 1979; Imura and Rockland, 2006; Nakamura et al., 2015). The widespread distribution of pulvinar dendritic arbors may explain why SC cells are transynaptically labeled after pseudorabies virus injections in the middle temporal cortical area (Lyon et al., 2010), even though tectopulvinar terminals do no overlap the distribution of pulvinar somata labeled by retrograde tracer injections in the same cortical regions (Stepniewska et al., 1999). The distribution of pulvinar neuron dendritic arbors suggests that a substantial integration of inputs may occur even when the distributions of pulvinar afferents are largely segregated. For example, the dendritic fields of individual mouse pulvinar neurons can extend across both the Pcm and Pl (Figure 17C, D), potentially receiving input from bilateral and ipsilateral tectopulvinar projections (Figure 13A, D), V1 (Figure 16K, 7A), as well as extrastriate cortical areas (Figure 16C, G). Therefore, it may be challenging to identify subclasses of pulvinar neurons based on presynaptic inputs. 
Comparison of neurons recorded within tectorecipient and striate-recipient zones of the cat pulvinar complex have revealed differences in receptive field sizes, direction- and orientation selectivity (Chalupa et al., 1983; Abramson and Chalupa, 1988; Chalupa and Abramson, 1988, 1989). However, analysis of spatiotemporal receptive field properties in these two zones using white noise and reverse correlation analysis suggests a significant integration of V1 and SC inputs across subdivisions (Piché et al., 2015). Furthermore, as discussed above, retrograde tracing techniques demonstrated that mouse pulvinocortical projections to V1 are coarsely topographic (Roth et al., 2016). However, this same study revealed that individual pulvinocortical boutons are activated by widely dispersed locations across the visual field, suggesting that while pulvinocortical axon projections may be aligned with the retinotopic organization of V1, they can contribute a surround modulation of cortical neurons that extends well beyond what their anatomical topography might imply.

Again, the mouse may be a useful model to dissect potential structure/function relationships within the pulvinar. Transgenic mouse lines (e.g. calretinin-cre) may provide a starting point for subdividing neuron groups, and whole cell recordings may identify differences in membrane properties (Monckton and McCormick, 2002; Li et al., 2003a; Ramcharan et al., 2005; Wei et al., 2011a). However, perhaps the most important step in this process is the characterization of pulvinar receptive field properties in moving animals, as discussed below.

Pulvinar activity and visual context 
In the anesthetized mouse, spontaneous activity in the pulvinar is significantly lower than that recorded in the dLGN (Roth et al., 2016), and even in awake but inactive primates, the spontaneous activity of pulvinar neurons is less than half that of dLGN neurons (Ramcharan et al., 2005). In addition, in anesthetized mice the proportion of pulvinar neurons that respond to simple visual stimuli is approximately half that of dLGN neurons (Allen et al., 2016). These differences in activity levels/visual responsiveness likely reflect functional distinctions between these two visual pathways. Recently, imaging studies in actively-moving mice have demonstrated that pulvinocortical projections to V1 signal discrepancies between optic flow and running speed (Roth et al., 2016). A similar role for the pulvinar in visuomotor coupling is supported by primate studies, where inactivation of the pulvinar nucleus disrupts the planning of visually-guided eye and hand movements (Wilke et al., 2010). Thus, the activity of the pulvinar nucleus reflects vision in the context of movement, and this activity appears to be critical for the subsequent planning and execution of appropriate visually-guided action.

Given this evidence, it appears to be essential to characterize pulvinar receptive field properties in the context of movement. To accomplish this, experiments must be carried out in awake behaving animals. While across-species comparative studies are needed, mice can be used to efficiently address a number of initial open questions. For example, what is the source of the motor signals in the pulvinar nucleus? It has been established that premotor cells in the deep SC provide corollary discharge signals to the mediodorsal nucleus to signal impending 
movements (Bickford and Hall, 1989; Sommer and Wurtz, 2002; Wurtz et al., 2011). In vitro slice studies have shown premotor cells in the deep layers of the SC can affect the activity of tectothalamic cells in the superficial layers (Phongphanphanee et al., 2011); in this way WFV cells could potentially provide contextual signals to the pulvinar nucleus. Recordings from WFV cells in awake behaving mice could determine whether internally-generated movement commands modify their responses to moving visual stimuli.

The pulvinar projects directly to the striatum and amygdala (discussed above), and preliminary studies indicate that pulvinocortical terminals target corticostriatal and corticoamygdala cells (Zhou et al., 2016). Thus, the pulvinar is at the center of a hub connecting the cortex, striatum and amygdala (Figure 19). The interconnected nature of these circuits (as well as their potential influence on SC circuits via the substantia nigra and/or zona incerta; Bickford and Hall, 1992; Kim et al., 1992; McHaffie et al., 2005), suggests that the pulvinar actively participates in the dynamic coordination of body movements with the perception of visual signals. However, it is still unclear how activity levels in the striatum and amygdala might affect pulvinar activity. Recording visual receptive field properties of pulvinar neurons during optogenetic manipulation of the amygdala (Tye et al., 2011; Wei et al., 2015), or subpopulations of striatal projection cells (Kravitz et al., 2012), may help to reveal mechanisms that impart context to pulvinar signals. 


\section{Summary}

Many similarities have been identified in the organization of the pulvinar nucleus across species, and the mouse provides a very useful model to continue to unravel the function of this puzzling structure. The tectorecipient pulvinar forms interconnected loops with the cortex, striatum and amygdala, and emerging evidence suggests that these circuits may be designed to code visual signals in the context of ongoing movement. Thus, the pulvinar nucleus may play a key role in the planning and execution of appropriate visually-guided movements, which require the precise coordination of perception and action. Future studies designed to manipulate circuits may shed light on the repertoire or behaviors mediated by the pulvinar nucleus, and mechanisms underlying their selection. In this way, the mouse model may be a particularly useful tool to inform and guide our understanding of the human pulvinar nucleus. 


\section{Figure 11. The pulvinar nucleus contains two tectorecipient zones}

Schematic illustrations indicate regions of the pulvinar nucleus in the mouse, squirrel, tree shrew, galago and macaque monkey that have been shown to receive dense convergent input (brown) or less dense topographic projections (peach) from the superior colliculus. The non-tectorecipient zones of the pulvinar are indicated in blue, and the location of the dorsal lateral geniculate nucleus (dLGN, gray) is indicated for reference. Illustrations are not to scale (adapted from Stepniewska et al., 2000;

Chomsung et al., 2008; Baldwin et al., 2011, 2013; Day-Brown et al., 2017).

Subdivisions for Mouse: Pcm, caudal medial pulvinar, Pl, lateral pulvinar, Prm, rostral medial pulvinar, Squirrel: C, caudal pulvinar, RL, rostral lateral pulvinar, RLm, medial rostral lateral pulvinar, RLl, lateral rostral lateral pulvinar, RM, rostral medial pulvinar, Tree shrew: Pc, central pulvinar, Pd, dorsal pulvinar, Pv, ventral pulvinar, Galago and Macaque: PIcm, central medial inferior pulvinar, PIcl, central lateral inferior pulvinar, PIp, posterior inferior pulvinar, PIpl, posterior lateral inferior pulvinar, PL, lateral pulvinar, PM, medial pulvinar, Macaque: PIm, medial inferior pulvinar. 

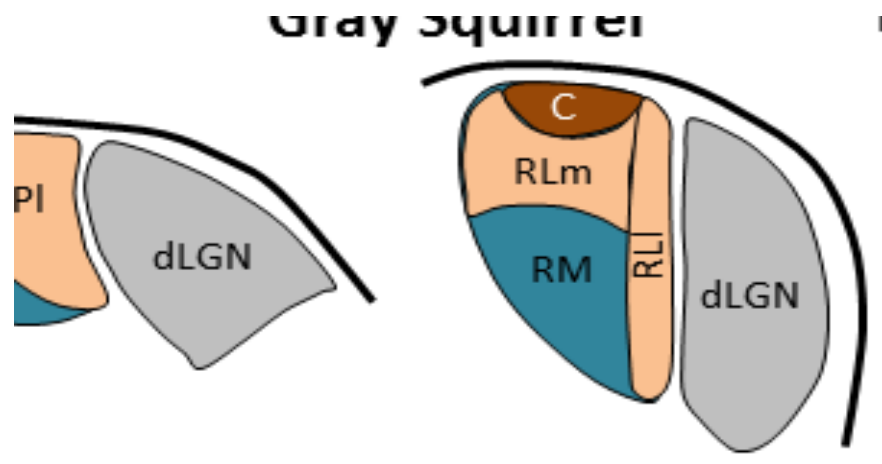

I ICC MIICvy

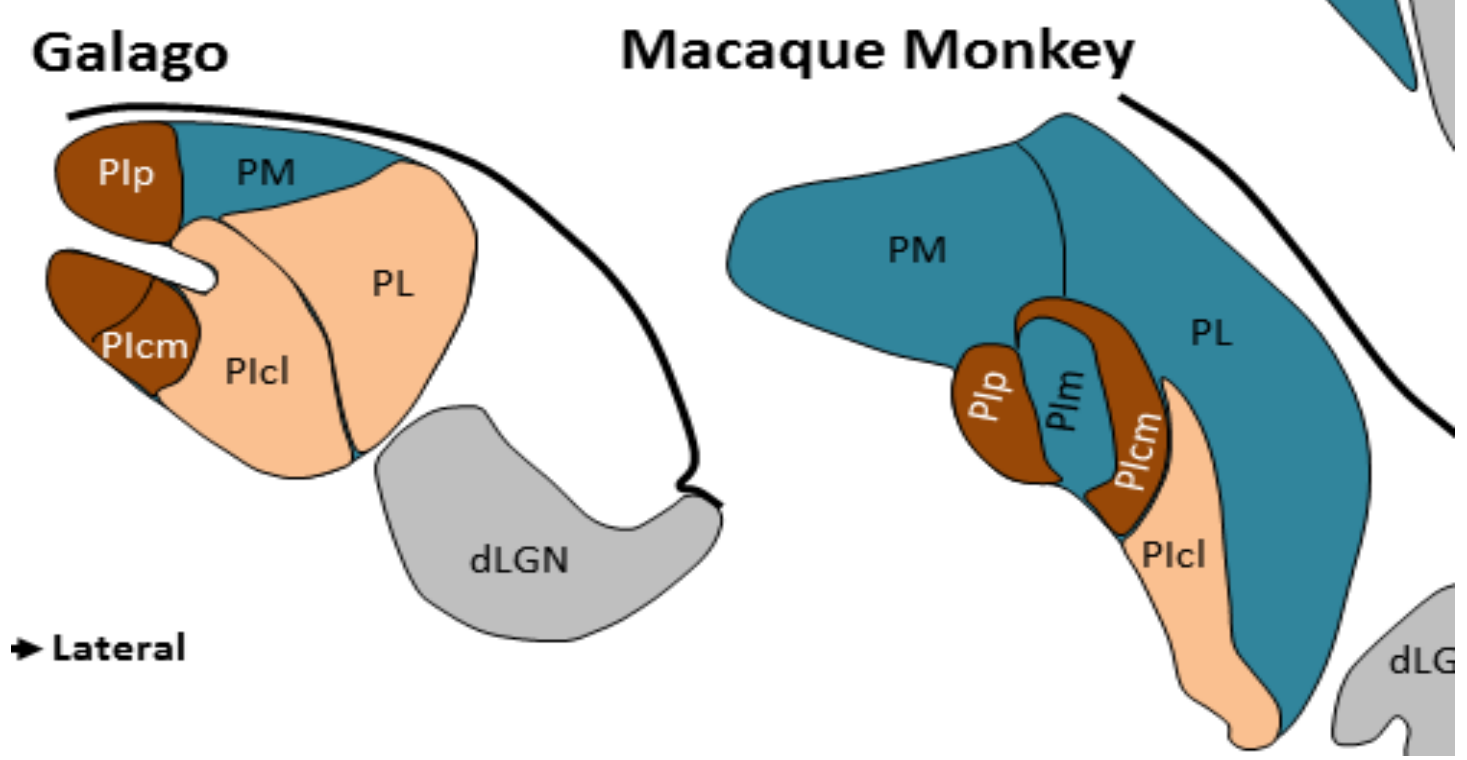


Figure 12. Widefield vertical (WFV) cells project to the ipsilateral and

\section{contralateral pulvinar}

Panel A illustrates an injection of a retrogradely transported virus (MIT viral vector core: hEF1 $\alpha$-EYFP-IRES-cre) in the pulvinar (PUL) of a wild type mouse that induced the expression of yellow fluorescent protein (YFP, green) in WFV cells of the superior colliculus (SC). Cells labeled by this injection are illustrated in panel B in a contralateral SC section that was stained with an antibody against calretinin (purple), which delineates the stratum griseum superficiale (SGS). The WFV tectopulvinar cells are located in the stratum opticum (SO) and lower SGS and extend dendrites to the surface of the SC, where they end in complex dendritic tufts (panel C). Panels D and E illustrate WFV cells labeled by injections of retrogradely transported cre-dependent viruses (MIT-viral vector core: hEF1 $\alpha$-LS1L-mCherry and hEF1 $\alpha$-LS1L-EYFP) in the left and right pulvinar of a substance P-cre mouse (Jackson Labs stock number 021877) to induce the expression of either YFP (green, left pulvinar injection) or mCherry (purple, right pulvinar injection) in cre-expressing neurons. Many WFV cells expressed both YFP and mCherry (white), demonstrating that a subpopulation of WFV cells bilaterally innervate the pulvinar, and that WFV cells express substance P. Scale bars: A and B = $100 \mu \mathrm{m}, \mathrm{C}=10 \mu \mathrm{m}, \mathrm{D}=50 \mu \mathrm{m}$ and also applies to $\mathrm{E}$. dLGN, dorsal lateral geniculate nucleus, PT, pretectum, OT, optic tract. Virus injection methods as in Bickford et al. (2015). 


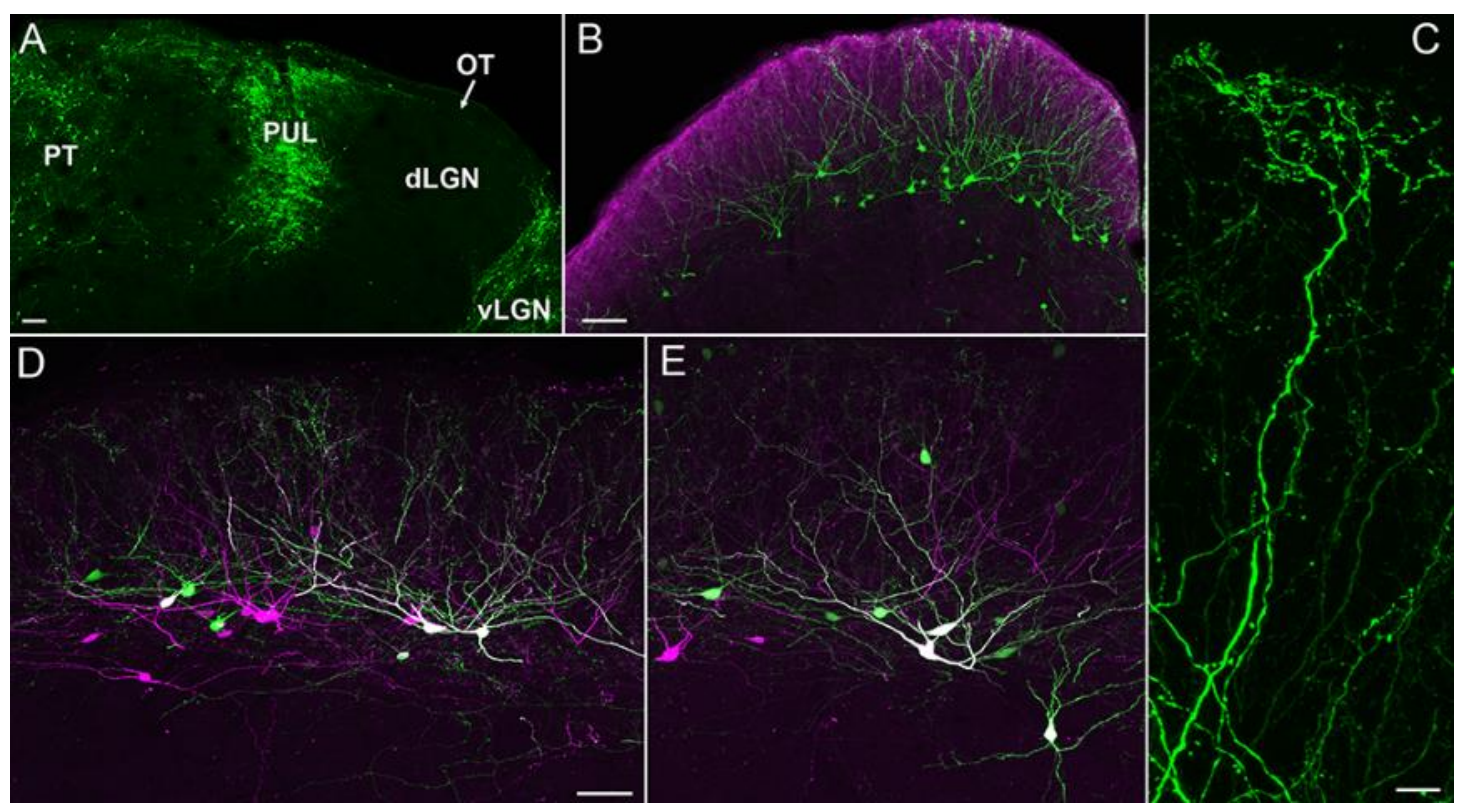


Figure 13. Caudal medial pulvinar ( $\mathrm{Pcm})$ cells express calretinin (CR) and neurokinin 1 (NK1) and align with bilateral superior colliculus (SC) projections Confocal images illustrate ipsilateral (A, C, green) and contralateral (D, F, green) projections to the pulvinar that were labeled by a unilateral virus injection in the SC. These sections were also stained with antibodies against CR (B, E, purple) to define the Pcm (which contains $\mathrm{CR}$ ) and the lateral pulvinar (Pl, which does not contain $\mathrm{CR}$ ). Adjacent sections (C, F) stained for CR (purple) and NK1 (green) illustrate that CRpositive Pcm cells express NK1. This expression pattern is shown at higher magnification in half micron optical sections in panels $\mathrm{G}(\mathrm{CR}$, purple), $\mathrm{H}(\mathrm{NK} 1$, green) and I(CR, purple, and NK1, green, asterisks indicate cells labeled with both antibodies). Scale in $A=50 \mu \mathrm{m}$ and applies to A-F. Scale in $\mathrm{G}=10 \mu \mathrm{m}$ and applies to G-I. dLGN, dorsal lateral geniculate nucleus, OT, optic tract, PT, pretectum. Methods as in Bickford et al. (2015) and Masterson et al. (2010). 


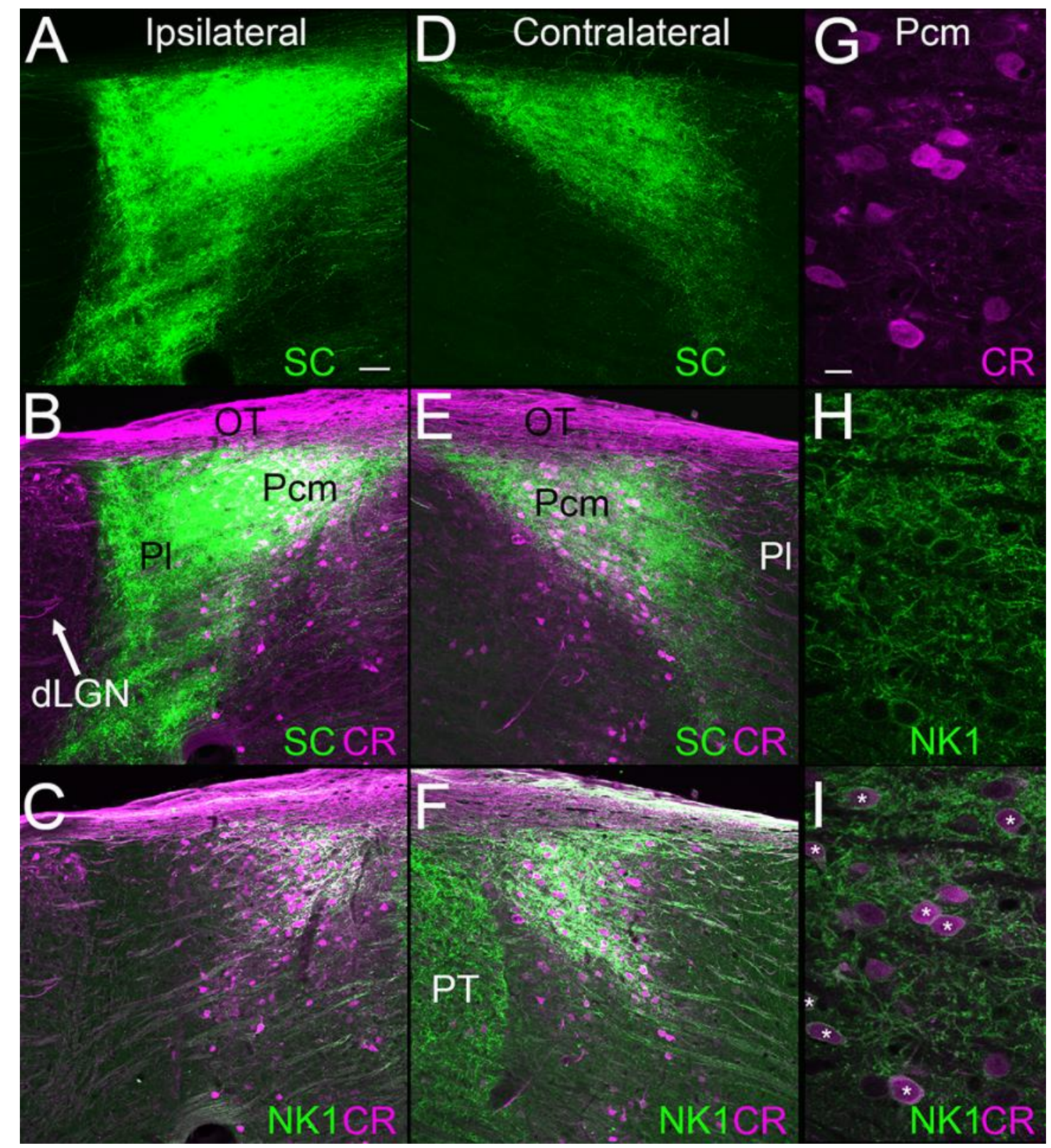


Figure 14. The Pcm contains a dense population of terminals that contain substance $\mathbf{P}$

A-C) Caudal to rostral sections stained with an antibody against substance P (visualized with a diaminobenzidine reaction). Staining is densest in the caudal and medial pulvinar $(\mathrm{Pcm})$. Little staining is observed in the lateral pulvinar $(\mathrm{Pl})$. Scale $=100 \mu \mathrm{m}$ and applies to all panels. dLGN, dorsal lateral geniculate nucleus, MGN, medial geniculate nucleus, OT, optic tract, PT, pretectum. Methods as in Masterson et al., 2010. 


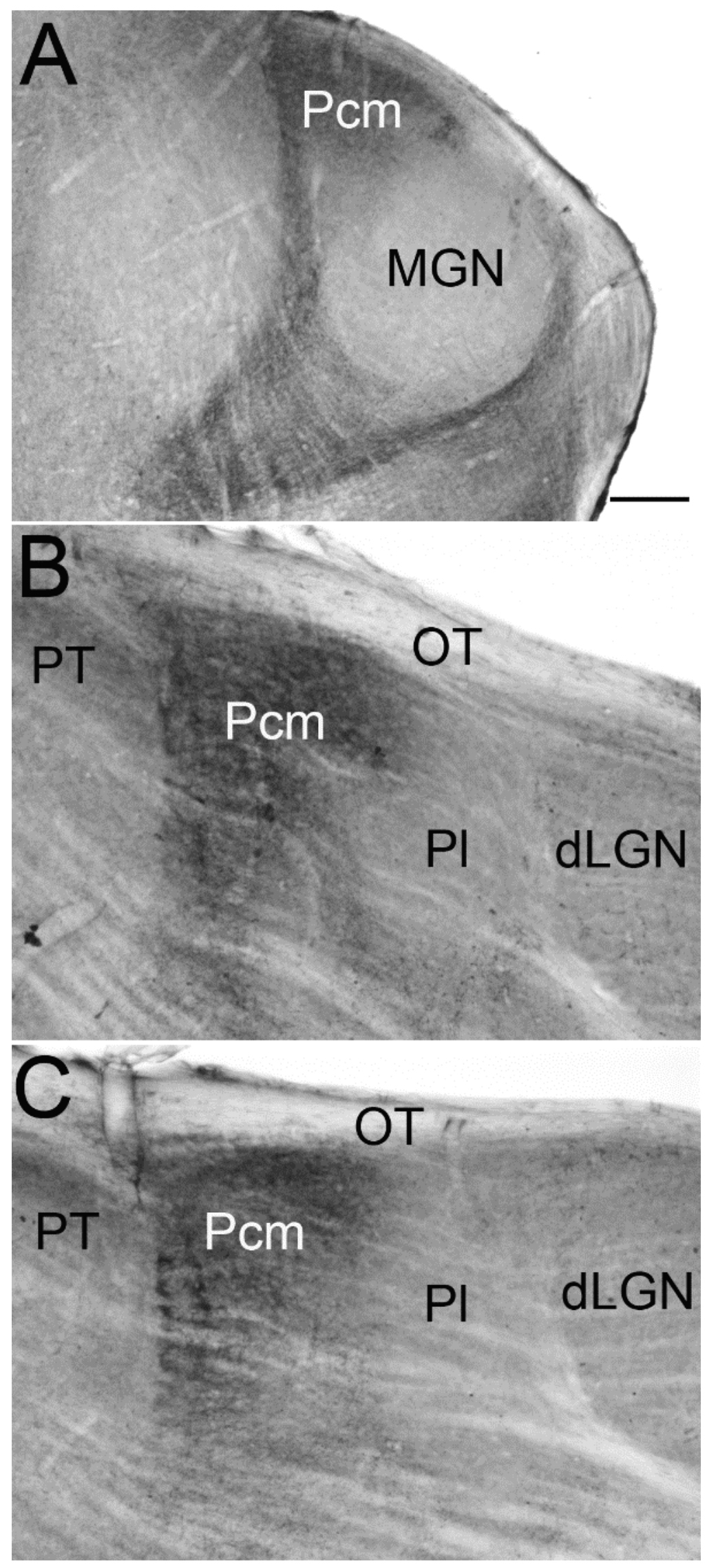


Figure 15. Ultrastructure of cortical and tectal terminals in the mouse pulvinar Terminals labeled by the anterograde transport of biotinylated dextran amine injected in V1 (A), superior colliculus (B) or the posterior/postrhinal cortex (C) contact (white arrows) the proximal (A, B) and distal (C) dendrites of pulvinar neurons (green overlay). Sections were additionally stained with gold particles to reveal the distribution of GABA. This indentifies two types of GABAergic terminals (purple overlay) in the mouse pulvinar: F2 profiles (B) contain a low density of vesicles and F1 profiles (C) contain a high density of vesicles. Scale $=600 \mathrm{~nm}$ and applies to all panels. Methods as in Li et al. (2003c). 


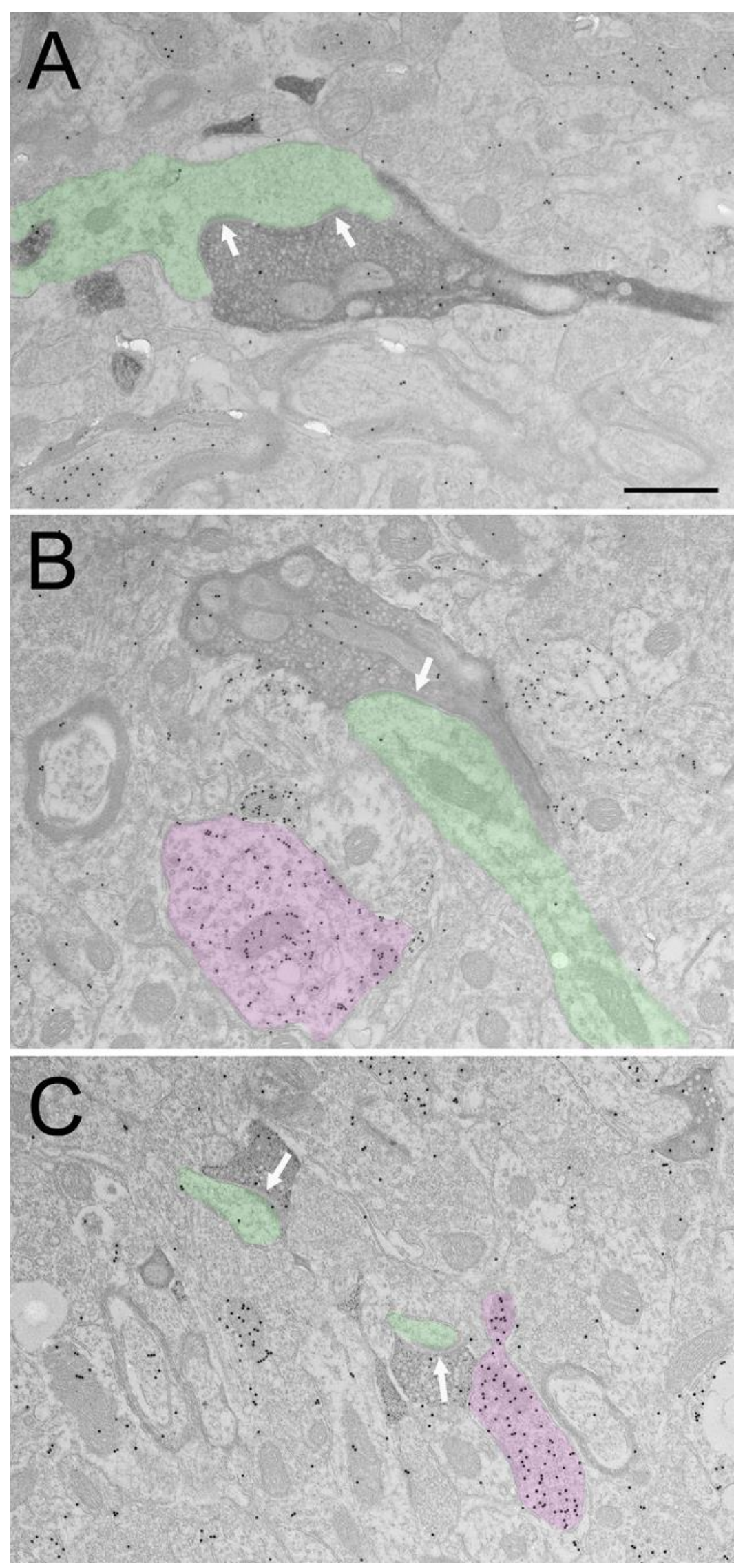


Figure 16. Tectopulvinar and corticopulvinar terminals overlap in the caudal medial (Pcm) and lateral (PI) subdivisions of the mouse pulvinar

This overlap is demonstrated via dual virus injections in the superior colliculus (SC) and lateral extrastriate cortex (LES, first 2 columns, A-D and E-H), or SC and V1 (last column, I-L). The Pcm and Pl subdivisions are defined using immunocytochemical staining for calretinin (CR, blue, first row, A, E, I). Virus injections were placed in the $\mathrm{SC}$ to induce the expression of yellow fluorescent protein (green, panels B, F, J), and in the cortex (V1 or LES) to induce the expression of TdTomato (red, panels C, G, K), Overlap of the CR and virus labeling patterns (panels D, H, L) show that the Pcm is innervated by the SC and LES, while the Pl is innervated by the SC, V1 and LES (panels D, H, L). Scale bar in D $=100 \mu \mathrm{m}$ and applies to all panels. dLGN, dorsal lateral geniculate nucleus, PT, pretectum. Methods as in Bickford et al. (2015) and Jurgens et al. (2012). 


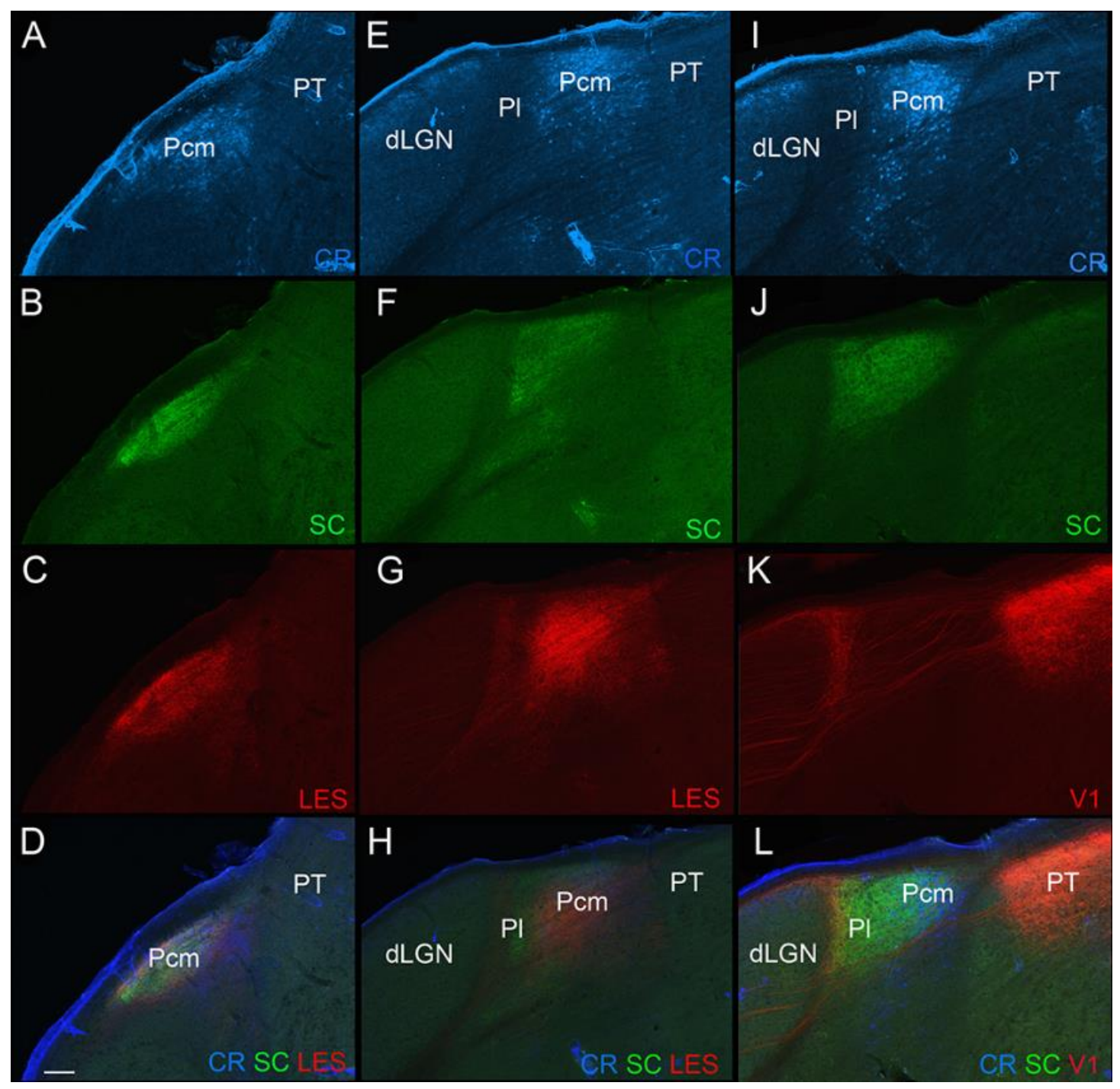




\section{Figure 17. Potential input integration in the mouse pulvinar}

Terminals labeled by a virus injection in V1 (green, A, B) and the ipsilateral SC (purple, B) overlap in the Pl. C) Two biocytin-filled pulvinar neurons (green) and surrounding tectopulvinar terminals (purple, labeled by a virus injection in the ipsilateral SC). The dendrites of the pulvinar neurons extend across subdivisions. D) Biocytin-filled pulvinar interneurons (purple) identified in a mouse line (Jackson Laboratories stock number 007677) that expresses green fluorescent protein in GABAergic neurons (green) extend dendrites across subdivisions. Scale bars $=20$ $\mu \mathrm{m}$. Methods as in Bickford et al., (2015). 


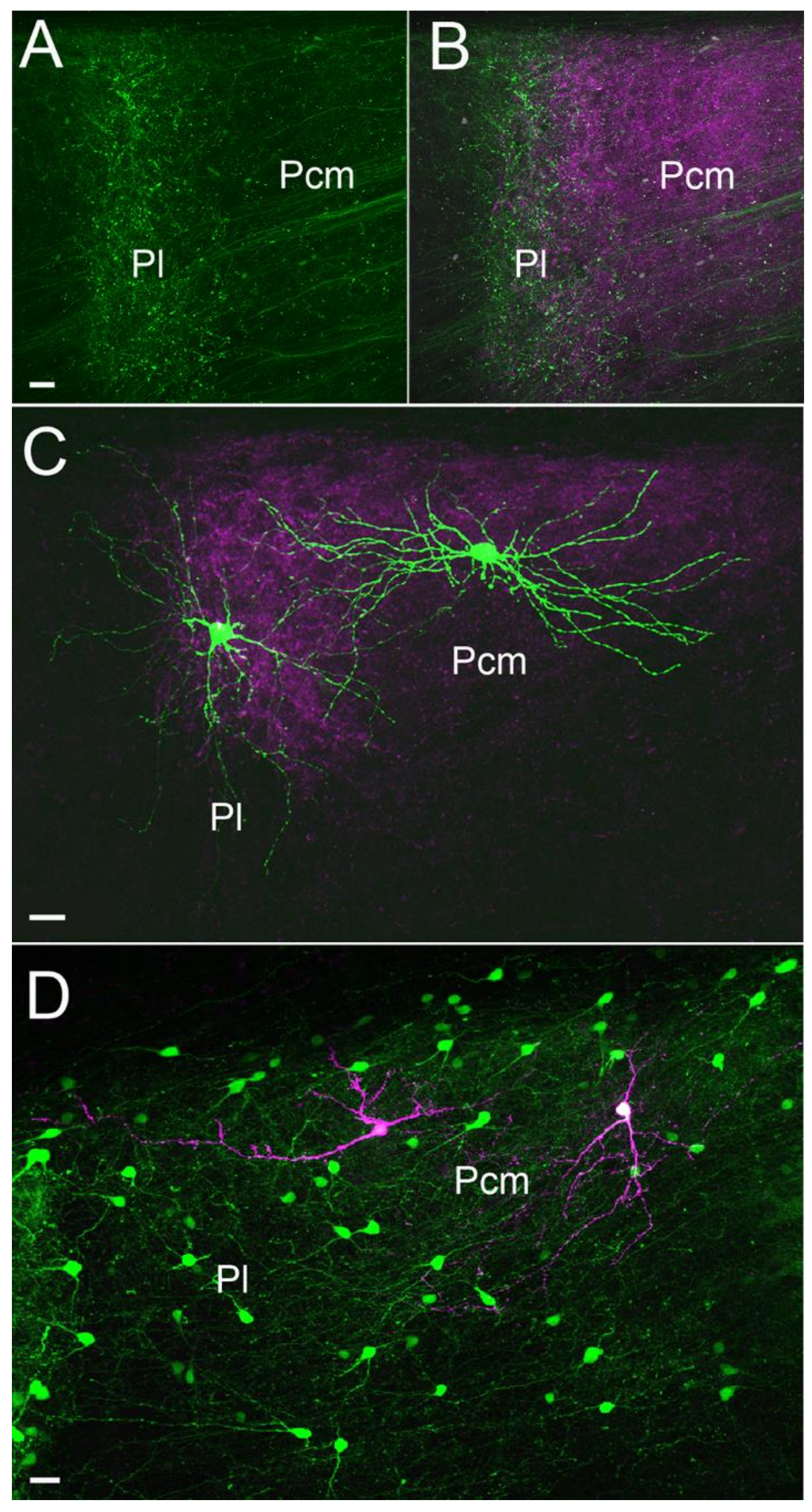


Figure 18. The mouse pulvinar projects to the cortex, striatum and amygdala Injections of biotinylated dextran amine in the mouse pulvinar (A) label terminals in V1 (B) and extrastriate cortex regions including the posterior medial area (PM, panel B) and the lateral medial area (LM, panel D). Cells in the superior colliculus (C) and LM (D) are also labeled by retrograde transport. E) The pulvinar also projects to the caudate and putamen $(\mathrm{CPu})$ and lateral amygdala (LA). Scale $=200 \mu \mathrm{m}$ and applies to all panels. Methods as in Chomsung et al., 2010. 


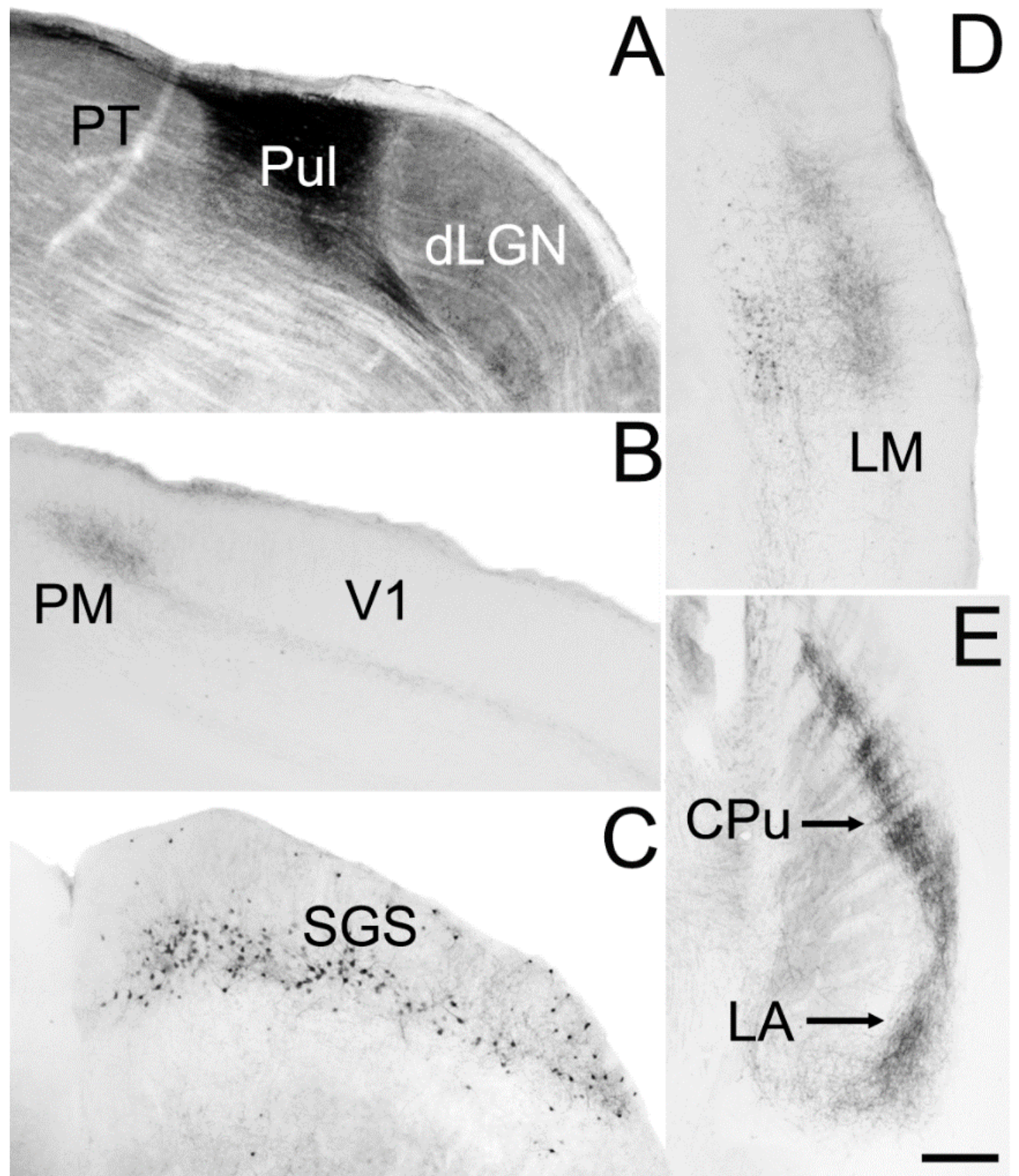


Figure 19. The tectorecipient mouse pulvinar forms interconnected loops with the cortex, striatum and amygdala

The schematic diagrams illustrate the main connections of the tectorecipient subdivisons of the mouse pulvinar. The caudal medial pulvinar (Pcm, red) receives bilateral input from widefield vertical (WFV) cells of the superior colliculus, and is reciprocally connected to the posterior $(\mathrm{P})$ and postrhinal (POR) regions of the cortex, where it innervates layers I and IV-VI. Both the Pcm and P/POR project to the caudal caudate/putamen $(\mathrm{CPu})$ and lateral amygdala $(\mathrm{LA})$. The lateral pulvinar ( $\mathrm{Pl}$, blue $)$ receives ipsilateral input from WFV cells, and is reciprocally connected to V1 and the lateral medial (LM) and lateral intermediate (LI) regions of the cortex. Within V1, the $\mathrm{Pl}$ projects to layers I and $\mathrm{Va}$. Within $\mathrm{LM}$ and $\mathrm{LI}$, the Pl projects to layer I and IV. The Pl, LM and LI project to the middle regions of the $\mathrm{CPu}$. 
Caudal medial pulvinar

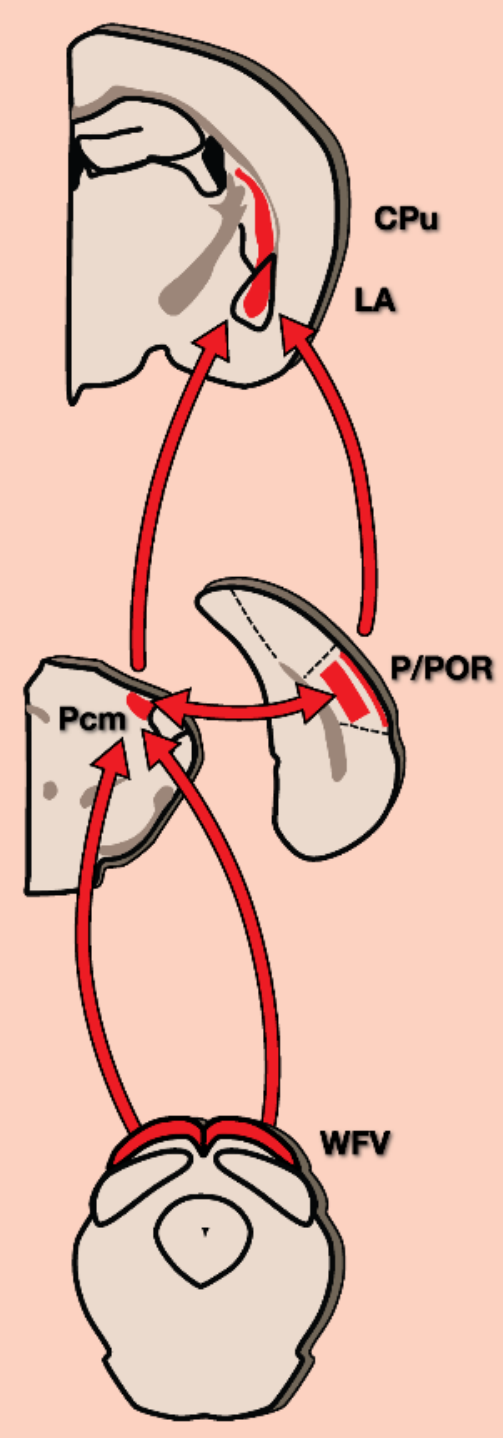

Lateral pulvinar

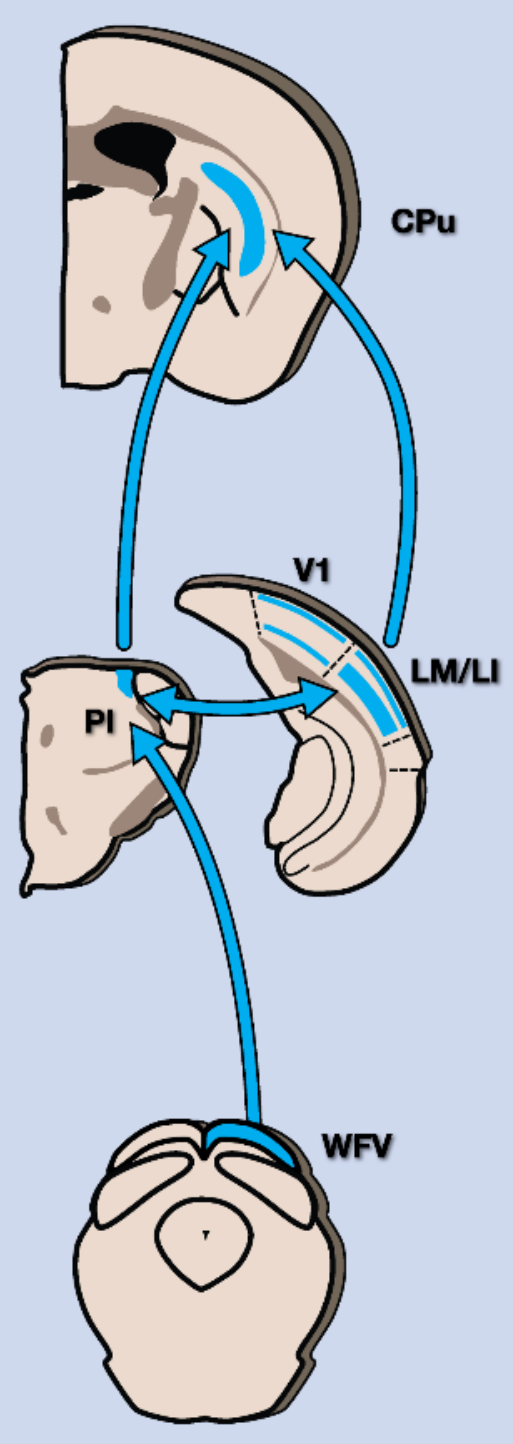

98 


\section{CHAPTER IV}

THE MOUSE PULVINAR NUCLEUS LINKS THE LATERAL EXTRASTRIATE CORTEX, STRIATUM, AND AMYGDALA: CELL TYPE-SPECIFIC THALAMOCORTICAL CIRCUIT FEATURES ${ }^{3}$

\section{Overview}

The pulvinar nucleus is a large thalamic structure involved in the integration of visual and motor signals. The pulvinar forms extensive connections with striate and extrastriate cortical areas, but the impact of these connections on cortical circuits has not previously been directly tested. Using a variety of anatomical, optogenetic, and in vitro physiological techniques in mice, we show that pulvinocortical terminals are densely distributed in the extrastriate cortex where they form synaptic connections with spines and small diameter dendrites. Optogenetic activation of these synapses in vitro evoked large excitatory postsynaptic responses in the majority of pyramidal cells, spiny stellate cells, and interneurons within the extrastriate cortex. However, specificity in pulvinar targeting was revealed when recordings were targeted to projection neuron subtypes. The neurons most responsive to pulvinar input were those

3. Zhou N, Masterson SP, Damron JK, Guido W, Bickford ME, submitted to Journal of Neuroscience $5-10-17$ 
that project to the striatum and amygdala (76\% responsive) or V1 (55\%), while neurons that project to the superior colliculus were rarely responsive $(6 \%)$. Because the pulvinar also projects directly to the striatum and amygdala, these results establish the pulvinar nucleus as a hub linking the visual cortex with subcortical regions involved in the initiation and control of movement. We suggest that these circuits may be particularly important for coordinating body movements and visual perception, and disruption of this network in humans may lead to visual neglect symptoms.

\section{Significance Statement}

We found that the pulvinar nucleus can strongly influence extrastriate cortical circuits and exerts a particularly strong impact on the activity of extrastriate neurons that project to the striatum and amygdala. Our results call into question the conventional hierarchical view of visual cortical processing, and in addition establish the pulvinar nucleus as a hub linking the visual cortex with subcortical regions involved in the initiation and control of movement. The execution of visually guided movements may rely on this network, since damage to homologous regions in human patients can produce symptoms of visual neglect.

\section{Introduction}

Vision is a very active process in that we constantly scan our surroundings using eye and body movements. As a consequence, there must be a tight coordination between the visual and motor circuits of the brain. A variety of studies suggest that the dorsal thalamus is involved in this visuomotor coupling. For example, in the primate, inactivation of the pulvinar nucleus disrupts the planning of visually-guided eye and 
hand movements (Wilke et al., 2010), and inactivation of the mediodorsal nucleus can both disrupt sequential saccadic eye movements, and shift visual receptive fields in the frontal cortex (Sommer and Wurtz, 2002, 2006). Most recently, projections to the striate cortex from the mouse pulvinar have been shown to signal discrepancies between optic flow and running speed (Roth et al., 2016). Therefore, pulvinar activity conveys visual information that relates to movement, and this activity appears to be crucial for the subsequent planning and execution of appropriate visually-guided actions. However, the synaptic mechanisms underlying these complex functions are currently unknown.

In particular, the impact of pulvinar projections on cortical circuits has not been directly tested. In fact, conventional hierarchical views of cortical organization imply a relatively minor impact of pulvinar projections on activity in extrastriate regions (Van Essen, 2005). Instead, corticocortical transfer of visual information from $\mathrm{V} 1$ is thought to drive receptive field properties in extrastriate regions of the visual cortex, because lesions of the striate cortex greatly diminish visually-driven activity in these areas (Girard and Bullier, 1989; Girard et al., 1991, 1992; Kaas and Krubitzer, 1992; Collins et al., 2003, 2005). Nevertheless, visually evoked activity can still be recorded in extrastriate areas following lesions or cooling of the striate cortex (Rodman et al., 1989; Girard et al., 1992; Azzopardi et al., 2003). Moreover, inactivation of the pulvinar nucleus can suppress visual activity within V1 (Purushothaman et al., 2012). Thus, pulvinar projections could effectively regulate cortical processing. 
In the current study, we sought to directly test the impact of pulvinar projections on cortical circuits by examining the synaptic properties of terminals that originate from this thalamic region. Using a combination of anatomical and optogenetic techniques, we found that the pulvinar nucleus can greatly impact the activity of the extrastriate cortex, and recordings targeted to specific projection neuron subtypes suggest that the pulvinar may act as a hub to dynamically coordinate body movements with the perception of visual signals.

\section{Materials and Methods}

\section{Animals}

All breeding and experimental procedures were approved by the University of Louisville Institutional Animal Care and Use Committees. Experiments were carried out using mice, of either sex, of a C57/BLK6 line, or a line in which neurons that contain the 65KD isoform of glutamic acid decarboxylase (GAD65) express green fluorescent protein (GFP; López-Bendito et al., 2004). A calretinin-cre driver line (Calb2-IRES-Cre, Jackson Labs stock number 010774, B6(Cg)-Calb2 ${ }^{\text {tml(cre)Zjh } / J) ~ w a s ~}$ used to generate a map of pulvinocortical projections.

\section{Biotinylated dextran amine (BDA) injections}

To label thalamocortical axon projections via anterograde transport, C57/BLK6 mice ranging in age between postnatal day (P) 22-35 were deeply anesthetized with a mixture of ketamine (100-150 mg/kg) and xylazine (10-15 $\mathrm{mg} / \mathrm{kg})$. The analgesic meloxicam (1-2 $\mathrm{mg} / \mathrm{kg})$ was also injected prior to surgery. The animals were then placed in a stereotaxic apparatus (Angle Two Stereotaxic, Leica, 
Wetzlar, Germany). An incision was made along the scalp, and a small hole was drilled in the skull. A glass pipette $(20-40 \mu \mathrm{m}$ tip diameter) containing a 5\% solution of biotinylated dextran amine (BDA, Molecular Probes) in saline was lowered into the dLGN (from Bregma: 2.14 posterior, 2.0 lateral, 2.89 ventral) or pulvinar (from Bregma: 2.11 posterior, 1.73 lateral, 2.73 ventral), and BDA was iontophoretically ejected using $3 \mu \mathrm{A}$ continuous positive current for 20 minutes. After removal of the pipette, the scalp skin was sealed with tissue adhesive (n-butyl cyanoacrylate), lidocaine was applied to the wound, and the animals were placed on a heating pad until mobile. Post-surgery, animals were carefully monitored for proper wound healing, and oral meloxicam (1-2 mg/kg) was administered for $48 \mathrm{~h}$.

\section{Cholera toxin subunit B (CTB) injections}

To label cortical projection cells via retrograde transport, P22-35 C57/BLK6, mice were prepared as described above. Either a glass pipette $(20-40 \mu \mathrm{m}$ tip diameter), or a Nanofil syringe with an attached 34 gauge needle, containing a $0.2 \%$ solution of cholera toxin subunit B conjugated to Alexafluor 488, 546 or 633 (CTB488, CTB-546, CTB-633) in phosphate buffered saline (PBS; 0.01M phosphate buffer with $0.9 \% \mathrm{NaCl}$ ) was lowered into cortical area V1 (from Bregma: 4.02 posterior, 2.5 lateral, 1.81 ventral), striatum and/or amygdala (from Bregma: 1.55 posterior, 3.58 lateral, 4.5 ventral) or superior colliculus (from Bregma: 4.09 posterior, 0.68 lateral, 1.7 ventral). CTB was iontophoretically ejected using $3 \mu \mathrm{A}$ continuous positive current for 15 or 30 minutes, or $100-210 \mathrm{nl}$ was ejected at a rate of $25 \mathrm{nl} / \mathrm{minute}$ using 
an ultramicropump. After removal of the pipette or needle, the wound was closed and the animals were treated during recovery as described above.

\section{Herpes simplex virus (HSV) injections}

The HSV virus hEF1a-EYFP-IRES-cre (obtained from the Massachusetts Institute of Technology viral vector core) was injected into the striatum and/or amygdala to label projection cells in the LES via retrograde uptake. This virus induced the infected cells to express a yellow fluorescent protein (YFP). P22-60 C57/BLK6 mice were deeply anesthetized and prepared as described above and a Nanofil syringe and ultramicropump were used to deliver volumes of 100-250nl at a rate of $10 \mathrm{nl} / \mathrm{minute}$. The wound was then closed, and the animals monitored during recovery as described above.

\section{AAV injections}

To label and activate the projections of the pulvinar, an adeno-associated virus (AAV) serotype 2/1 carrying a vector for the Channelrhodopsin variant Chimera EF with $\mathrm{I} 170$ mutation (ChIEF) fused to the red fluorescent protein, tdTomato (production details in Jurgens et al., 2012) was injected unilaterally or bilaterally into the pulvinar. To label projections from the pulvinar to subsequently construct a map of cortical areas in the coronal plane, a cre-dependent virus, Flex-rev-oChIEFtdTomato (Plasmid \#30541, Addgene), packaged using AAV serotype 9, was injected unilaterally into the pulvinar of calretinin-cre mice. For virus delivery, P22-60 C57/BLK6 or GAD65-GFP mice were deeply anesthetized with a mixture of ketamine and xylazine as described above. An incision was made along the scalp, and 
a small hole created in the skull above the left and/or right pulvinar. Virus was delivered via a 34-gauge needle attached to a Nanofil syringe inserted in an ultramicropump. A volume of $75 \mathrm{nl}$ was injected into each pulvinar at a rate of 20nl/minute.

\section{AAV and CTB or HSV injections}

In some cases, in the same animals that received unilateral AAV injections in the pulvinar, an additional injection of CTB-488 or HSV-YFP was placed in the ipsilateral cortex, striatum/amygdala, or SC as described above. The wound was then closed, and the animals monitored during recovery as described above.

\section{Slice preparation and optogenetic stimulation}

Eight to 12 days following virus injections, mice were deeply anesthetized with avertin $(0.5 \mathrm{mg} / \mathrm{kg})$. Mice used for slice preparation ranged in age from P29-P37 (average age P31). Mice were either directly decapitated or transcardinally perfused with cold $\left(4{ }^{\circ} \mathrm{C}\right)$, oxygenated $\left(95 \% \mathrm{O}_{2} / 5 \% \mathrm{CO}_{2}\right)$ slicing solution containing the following (in mM): $2.5 \mathrm{KCl}, 26 \mathrm{NaHCO}_{3}, 2.5 \mathrm{KCl}, 1.25 \mathrm{NaH}_{2} \mathrm{PO}_{4}, 10 \mathrm{MgCl}_{2}, 2$ $\mathrm{CaCl}_{2}, 234$ sucrose, and 11 glucose, before rapid decapitation (in mice older than P35). The brain was removed from the head, chilled in the cold slicing solution described above for 2 mins, and was quickly transferred into a petri dish with room temperature slicing solution to block the brain for subsequent sectioning. Coronal slices $(300 \mu \mathrm{m})$ were cut in room temperature slicing solution using a vibratome (Leica VT1000 S). Then slices were transferred into an incubation solution of oxygenated $\left(95 \% \mathrm{O}_{2} / 5 \% \mathrm{CO}_{2}\right)$ artificial cerebrospinal fluid (ACSF) containing (in 
$\mathrm{mM}): 126 \mathrm{NaCl}, 26 \mathrm{NaHCO}_{3}, 2.5 \mathrm{KCl}, 1.25 \mathrm{NaH}_{2} \mathrm{PO}_{4}, 2 \mathrm{MgCl}_{2}, 2 \mathrm{CaCl}_{2}$, and 10 glucose at $32^{\circ} \mathrm{C}$ for 30 mins, and later maintained at room temperature.

Individual slices were transferred into a recording chamber, which was maintained at $32^{\circ} \mathrm{C}$ by an inline heater and continuously perfused with room temperature oxygenated ACSF $\left(2.5 \mathrm{ml} / \mathrm{min}, 95 \% \mathrm{O}_{2} / 5 \% \mathrm{CO}_{2}\right)$. Slices were stabilized by a slice anchor or harp (Warner Instruments 64-0252). Neurons were visualized on an upright microscope (Olympus BX51WI) equipped with both differential interference contrast optics and filter sets for visualizing CTB-488 and YFP (Chroma 49002) or tdTomato (Chroma 49005) using a 4x or 60x water-immersion objective (Olympus) and a CCD camera. Recording electrodes were pulled from borosilicate glass capillaries (World Precision Instrument Inc.) by using a MODEL P-97 puller (Sutter Instrument Co., Novato, CA). The electrode tip resistance was 4-6 $\mathrm{M} \Omega$ when filled with an intracellular solution containing the following (in $\mathrm{mM}$ ): $117 \mathrm{~K}$-gluconate, 13.0 KCl, $1 \mathrm{MgCl}_{2}, 0.07 \mathrm{CaCl}_{2}, 0.1$ EGTA, 10 HEPES, $2 \mathrm{Na}_{2}$-ATP, and 0.4 $\mathrm{Na}_{2}$-GTP with PH adjusted to 7.3 with $\mathrm{KOH}$ and osmolarity 290-295 mOsm. Biocytin (0.5\%) was added to this intracellular solution to allow morphological reconstruction of the recorded neurons.

Whole-cell recordings were obtained from the LES regions of the cortex. For single injection experiments (in which only the pulvinar was injected with virus), cells in layers IV and $\mathrm{V}$ were targeted for recording within the pulvinar termination zones. For GAD65-GFP experiments, interneurons labeled with GFP with overlapping pulvinar terminals were targeted for recording. For double injection experiments (in 
which the pulvinar was injected with virus and the SC, striatum or cortex were injected with CTB-488 or HSV-YFP to label LES projection cells via retrograde transport), labeled and unlabeled cells were targeted for recording with the pulvinar termination zones. Video images of the patched cell locations, and the CTB/YFP/GFP within patched cells, were recorded using the CCD camera.

Recordings were obtained with an AxoClamp 2B amplifier (Axon Instruments, Foster City, CA) and a Digidata 1440A was used to acquire electrophysiological signals. The stimulation trigger was controlled by Clampex 10.3 software (Molecular Devices). The signals were sampled at $20 \mathrm{kHz}$ and data were analyzed offline by pClamp 10.0 (Molecular Devices). Series resistance was compensated by a bridge protocol and only recordings with stable series resistance were included in the analysis. For current clamp recordings, voltage signals were obtained from cells with resting potential of $-60 \mathrm{mV}$ to $-75 \mathrm{mV}$. For voltage clamp recordings, membrane currents were obtained at $-65 \mathrm{mV}$ to $-75 \mathrm{mV}$.

For photoactivation of pulvinocortical terminals, light from a blue light emitting diode (Prizmatix UHP 460) was reflected into a 60X water immersion objective. This produced a spot of blue light onto the submerged slice with an approximate diameter of $0.3 \mathrm{~mm}$. Pulse duration and frequency were under computer control. For repetitive stimulation, pulse duration was either 1 or $10 \mathrm{~ms}$. Synaptic responses were recorded using light intensities of $10-112 \mathrm{~mW} / \mathrm{mm}^{2}$, and light pulse frequencies of $1 \mathrm{~Hz}, 2 \mathrm{~Hz}, 5 \mathrm{~Hz}, 10 \mathrm{~Hz}$ and $20 \mathrm{~Hz}$.

To test whether the responses were monosynaptic, tetrodotoxin (TTX, $1 \mu \mathrm{M}$; 
Alomone labs, catalogue \# T-550) was added to the bath to block action potentials and 4-aminopyridine (4-AP, 1mM; Sigma, catalogue \#275875-5G) was added to augment depolarization of the terminals. In other experiments, 2-amino-5-phosphonovaleric acid (APV,10 $\mu \mathrm{M}$; Sigma, catalogue \#A-5282) and/or 6-cyano-7-nitroquinoxalene2,3-dione (CNQX, 8$\mu \mathrm{M}$; Tocris, catalogue \#0190)/ 6,7- dinitroquinoxaline-2,3-dione (DNQX, $80 \mu \mathrm{M}$; Sigma, catalogue \#D0540-50MG) were added to the bath to block Nmethyl-D-aspartate (NMDA) and $\alpha$-amino-3-hydroxy-5-methyl-4-isoxazolepropionic acid (AMPA) receptors. Gamma amino butyric acid receptors $\left(\mathrm{GABA}_{\mathrm{A}}\right)$ were blocked via bath application of the antagonist 2-(3-carboxypropyl)-3-amino- 6-(4methoxyphenyl)-pyridazinium bromide (SR95531, 20 $\mu \mathrm{M}$; Tocris, catalogue \#1262).

\section{Processing of cells filled during physiological recording}

Following recording, slices were placed in a fixative solution of $4 \%$ paraformaldehyde in $0.1 \mathrm{M}$ phosphate buffer $\mathrm{pH} 7.4(\mathrm{~PB})$ for at least 24 hours. The sections were then rinsed in PB and incubated overnight in a 1:1000 dilution of streptavidin-conjugated to Alexafluor-633 (Invitrogen, Carlsbad, CA) in PB containing $1 \%$ Triton $\mathrm{X}-100$. The following day the slices were washed in PB, preincubated in $10 \%$ normal goat serum (NGS) in PB and then incubated overnight in a 1:500 dilution of a rabbit anti-DSred antibody (Clonetech Laboratories, Inc. Mountainview, CA, catalogue \#632496) in PB with 1\% NGS. The following day the sections were rinsed in $\mathrm{PB}$, and incubated for 1 hour in a 1:100 dilution of a goat-antirabbit antibody conjugated to Alexafluor-546 (Invitrogen). The sections were then rinsed in PB and mounted on slides to be imaged with a confocal microscope. 


\section{Histology for tissue used for anatomical analyses}

For animals that were not used for physiological experiments, 2 days to 2 weeks following injection of tracers and/or viruses, mice were deeply anesthetized with Avertin $(0.5 \mathrm{mg} / \mathrm{gm})$ and transcardially perfused with a fixative solution of $4 \%$ paraformaldehyde, or 2\% paraformaldehyde and 2\% glutaraldehyde in PB. Additional C57/BLK6 or GAD65-GFP mice that were not injected were also perfused for immunocytochemistry. In each case, the brain was removed from the skull and $70 \mu \mathrm{m}$ thick coronal sections were cut using a vibratome (Leica Microsystems, Buffalo Grove, IL). Sections that contained fluorescent labels were mounted on slides and imaged using a confocal microscope (Olympus FV1200BX61), or additionally stained using antibodies as described below.

Selected sections were incubated overnight in antibodies against parvalbumin (made in mouse, Sigma, catalogue P3088, 1:2000) or calretinin (made in mouse, Millipore, catalogue MAB1568, 1:1000). The following day, the sections were incubated in a 1:100 dilution of a goat-anti-mouse antibody that was directly conjugated to fluorescent compounds (Alexafluor 488, 546 or 633; Invitrogen, Carlsbad, CA). The sections were then mounted on slides and imaged using a confocal microscope (Olympus).

To label tissue for viewing in a transmitted light microscope or transmission electron microscope, sections that contained CTB-labeled cells were incubated overnight in a rabbit anti-CTB antibody (Sigma catalogue \# C3062; 1: 10,000). Sections that contained TdTomato were incubated overnight in a rabbit anti-DSred 
antibody (1:500). Sections that contained YFP or GFP were incubated overnight in a rabbit anti-GFP antibody (Millipore, Billerica, MA, catalogue \#AB3080, 1:1000). Sections incubated in the antibodies, or sections that contained BDA were incubated in a 1:100 dilution of a biotinylated goat-anti-rabbit antibody (1 hour), followed by avidin and biotinylated horseradish peroxidase (ABC solution, Vector Laboratories, 1 hour) and reacted with nickel-enhanced diaminobenzidine (DAB). The sections were then mounted on slides and imaged using transmitted light, or processed for electron microscopy as described below.

\section{Electron microscopy}

Sections that contained terminals labeled by the anterograde transport of BDA, or cells and terminals labeled with the GFP antibody, were postfixed in $2 \%$ osmium tetroxide, dehydrated in an ethyl alcohol series, and flat embedded in Durcupan resin between two sheets of Aclar plastic (Ladd Research, Williston, VT). Durcupanembedded sections were first examined with a light microscope to select areas for electron microscopic analysis. Selected areas were mounted on blocks, ultrathin sections (70-80 nm, silver-gray interference color) were cut using a diamond knife, and sections were collected on Formvar-coated nickel slot grids. Selected sections were stained for the presence of gamma amino butyric acid (GABA). A postembedding immunocytochemical protocol described previously (Chomsung et al., 2008, 2010; Day-Brown et al., 2010) was employed. Briefly, we used a rabbit polyclonal antibody against GABA that was tagged with a goat-anti-rabbit antibody conjugated to 15-nm gold particles (BBI Solutions USA, Madison, WI). The sections 
were air dried and stained with a $10 \%$ solution of uranyl acetate in methanol for 30 minutes before examination with an electron microscope.

\section{Experimental design and statistical analyses}

Three general types of experiments were developed and reported in this manuscript: 1) electron microscopic analysis of labeled profiles, 2) in vitro electrophysiological analysis of responses to photoactivation of pulvinocortical terminals and 3) morphological analysis of cortical neurons filled with biocytin during recording.

1) For electron microscopic analysis of tracer-labeled thalamocortical terminals and their postsynaptic targets ( $\mathrm{n}=7$ animals), ultrathin tissue sections were examined using an electron microscope and every labeled terminal involved in a synapse was imaged ( $\mathrm{n}=501$ terminals). The pre- and postsynaptic profiles were characterized on the basis of size (measured using Image J, RRID: nif-000-30467, Maxim DL (C) 5 software), and the presence or absence of synaptic vesicles. To analyze tissue from a GAD65-GFP mouse that was stained for GABA using postembedding immunocytochemical techniques, GFP-labeled profiles $(n=136)$ were imaged and the overlying gold particle density was quantified. One-way ANOVA with Tukey's multiple comparisons post-test were used for statistical analyses of ultrastructural data, and plotted as column box-and-whisker graphs using Prism 6.0.

2) For in vitro electrophysiological analysis of responses to photoactivation of pulvinocortical terminals in labeled or unlabeled neurons, 108 animals of either sex were used and a total of 501 neurons were patched in slices of the cortex (4-5 cortical 
slices were used per animal). The majority of recorded neurons $(n=425)$ were tested to determine their response to activation of surrounding pulvinocortical terminals. Baseline values were measured just prior to the onset of photostimulation and the amplitude of synaptic responses was measured from this baseline value. Further analysis was limited to neurons with response amplitudes $>7$ times the standard deviation of the baseline $(n=278)$. Neurons below this conservative threshold were considered nonresponsive and excluded from further electrophysiological analysis. Electrophysiological measurements were tested for normality using the D'AgostinoPearson omnibus test and appropriate statistical analyses were chosen based on the Gaussian or non-Gaussian distribution of the data. For each variable, differences between the groups were assessed using nonparametric one-way ANOVA followed by Post-hoc Tukey's multiple comparison test or nonparametric Kruskal-Wallis ANOVA test with Post-hoc Dunn's multiple comparison test. All data were presented as mean \pm SD except the vertical scatter plots which were plotted as mean \pm SEM. Significance was set at $\alpha=0.05$ for all statistical tests. Prism 6.0 was used to generate vertical scatter plots, bar graphs, column mean \pm SD connected graphs or grouped interleaved bar graphs.

3) Confocal images of labeled cells were categorized based on the following criteria: location of soma and dendrites relative to labeled thalamocortical terminals, location of the neuron within a map of the cortex (Figure 10), the presence or absence of an apical dendrite, or the presence or absence of markers in the soma (retrograde markers CTB or YFP, or GFP labeling in GAD65-GFP mice). A total of 356 cells 
were recovered, and the labeling of 316 of these cells was sufficiently complete to categorize their morphology.

\section{Results}

\section{Extrinsic projections of the mouse pulvinar: comparison with the dLGN}

Pulvinar and $d L G N$ projections to $V 1$

Iontophoretic injections of BDA in the mouse dLGN or pulvinar label distinct bands of terminals in V1. Terminals labeled by injections in the dLGN are concentrated in layers IV and I, with sparser terminations located in layers II/III and VI (Figure 20A). Terminals labeled by injections in the pulvinar are concentrated in layers I and Va (Figure 20B), as previously described (Herkenham, 1980; Roth et al., 2016). Electron microscopic analysis of labeled terminals involved in synapses (dLGN-V1 terminals in layer I $\mathrm{n}=84, \mathrm{dLGN}-\mathrm{V} 1$ terminals in layer IV $\mathrm{n}=94$, pulvinar-V1 terminals in layers 1 and Va, $\mathrm{n}=83$ ) revealed that $\mathrm{dLGN}-\mathrm{V} 1$ and pulvinar-V1 terminals contain densely packed synaptic vesicles, and make synaptic contacts on small dendrites and spines with thick postsynaptic densities (Figure 20D).

Measurements of the size of pre- and postsynaptic profiles (Figure 20G and $20 \mathrm{H})$ established that dLGN-V1 terminals in layer IV are significantly larger $(0.36 \pm$ $\left.0.19 \mu \mathrm{m}^{2}\right)$ than dLGN-V1 terminals in layer I $\left(0.28 \pm 0.15 \mu \mathrm{m}^{2}\right.$; One-way ANOVA with Tukey's post-test, $\mathrm{p}<0.05)$, and pulvinar-V1 terminals $\left(0.25 \pm 0.20 \mu \mathrm{m}^{2}\right)$ are significantly smaller than dLGN-V1 terminals in layer IV $(\mathrm{p}<0.01)$. However, we found no significant difference in the size of profiles postsynaptic to each terminal type (dLGN to layer I: $0.19 \pm 0.23 \mu \mathrm{m}^{2}$, dLGN to layer IV: $0.14 \pm 0.07 \mu \mathrm{m}^{2}$, pulvinar 
to layers I and Va: $0.14 \pm 0.06 \mu \mathrm{m}^{2}$ ).

\section{Pulvinar projections to LES}

In addition to $\mathrm{V} 1$, the pulvinar projects densely to the lateral extrastriate cortex (LES corresponding to the posterior, postrhinal, lateromedial, and laterointermediate cortex: P, POR, LM and LI, defined by Wang and Burkhalter 2007). Within the LM, LI regions, projections from the pulvinar are concentrated in layers IV and I (Figure 20C). In fact, the laminar distribution of pulvinocortical terminals in the LM and LI is very similar to that of geniculocortical terminals in V1 (Figure 20A). Pulvinar projections are most densely distributed within the P and POR. In these cortical regions, projections from the pulvinar are concentrated in layers I and IV, but also extend into layers V and VI (Roth et al., 2016). The ultrastructure of labeled terminals involved in synapses $(\mathrm{LM} / \mathrm{LI} \mathrm{n}=118, \mathrm{P} / \mathrm{POR} \mathrm{n}=122)$ was found to be similar to that of thalamocortical terminals in V1. In both $\mathrm{LM} / \mathrm{LI}$ and P/POR, terminals that originate from the pulvinar contain densely packed synaptic vesicles, and make synaptic contacts on small dendrites and spines with thick postsynaptic densities (Figure 20E and 20F). Measurements of the size of pre- and postsynaptic profiles (Figure 20G and $20 \mathrm{H})$ established that the size of pulvinar terminals in the LM/LI $\left(0.33 \pm 0.20 \mu \mathrm{m}^{2}\right)$ and P/POR $\left(0.35 \pm 0.18 \mu \mathrm{m}^{2}\right)$ regions are not significantly different than that of dLGN-V1 terminals in layer IV. However, pulvinar-P/POR terminals contact larger postsynaptic dendrites (P/POR $\left.0.21 \pm 0.14 \mu \mathrm{m}^{2}\right)$ than either those contacted by layer IV dLGN-V1 terminals $(\mathrm{p}<0.01)$ or those contacted by pulvinar-V1 terminals $(p<0.05)$. There is no significant difference between dendrites contacted by pulvinar- 
LM/LI and dLGN-V1 terminals (LM/LI $\left.0.17 \pm 0.10 \mu \mathrm{m}^{2}\right)$. Our ultrastructure measurements also showed that pulvinar-LES terminals are much larger than pulvinar-V1 terminals (P/POR: $\mathrm{p}<0.01 ; \mathrm{LM} / \mathrm{LI}: \mathrm{p}<0.05)$.

\section{Optogenetic activation of thalamocortical terminals that originate from the}

pulvinar

To activate thalamocortical terminals that originate from the pulvinar, viral vector injections were placed in the caudal and lateral parts of the pulvinar (Figure 21A-B) to induce the expression of TdTomato and Chief in pulvinar cells and their axon projections; this resulted in a dense band of terminals in the LES and sparser terminations in V1 (Figure 21C-E), similar to the patterns observed following BDA injections in the pulvinar (Figure 20B-C). In coronal slices of the cortex containing V1 and LES, whole cell recordings were obtained from neurons in the regions innervated by the pulvinar $(\mathrm{n}=501)$. The majority of these recorded neurons $(\mathrm{n}=$ 425) were tested to determine their response to activation of surrounding pulvinocortical terminals. The remaining recorded neurons were simply filled with biocytin to augment our morphological analysis of cell types in the extrastriate cortex.

Pulses of blue light ( 1 or $10 \mathrm{~ms}$ in duration) through the microscope objective were used to activate the light-sensitive channels expressed by the pulvinar terminals (Figure 21A). These induced robust responses, with short ( $<6.5 \mathrm{~ms})$, fixed latencies in the majority of neurons ( 278 of 425 or $65 \%$ of recorded neurons). The responses of neurons to activation of surrounding pulvinar terminals increased in amplitude as the intensity of the blue light pulses was increased (Figure $21 \mathrm{~F}$ left), and action potentials 
could be elicited in postsynaptic neurons with light intensities as low as $\left(14 \mathrm{~mW} / \mathrm{mm}^{2}\right.$, Figure $21 \mathrm{~F}$ left).

To ensure that activation of pulvinar terminals elicited monosynaptic responses, the sodium channel blocker TTX was added to the bath to block action potentials, paired with the potassium channel blocker 4AP to enhance the depolarization of the terminals $(n=38)$. In the presence of TTX and 4AP, large amplitude responses could be elicited (Figure $21 \mathrm{~F}$ right), which were abolished by subsequent application of the AMPA and NMDA receptor antagonists CNQX and $\operatorname{APV}(\mathrm{n}=12 ;$ Figure 21F right $)$.

\section{Morphology and location of recorded cortical neurons}

Pipettes included biocytin so that the location and morphology of recorded cells could be established after recording (Figure 21G; 356 cells were recovered, and the labeling of 316 of these cells was sufficiently complete to categorize their morphology). We found that the majority of these (220 of 316 or $70 \%$ ) were pyramidal cells, characterized by the presence of apical dendrites that extended from the soma toward the cortex surface (Figure 22C-E). Pyramidal cells were further subdivided based on the branching patterns of their apical dendrites. Most pyramidal cells had a single apical dendrite (157 of 220 or $71 \%$ ). Other pyramidal cells had two or three apical dendrite branches, in which each branch made up greater than half the total length of the apical arbor (two branches, 58 of 220 or $26 \%$, three branches, 5 of 220 or $2 \%$ ). The remaining recovered cells were either spiny stellate cells (51 of 316 or $16 \%$ ), characterized by radially oriented spiny dendrites (Figure $23 \mathrm{C}-\mathrm{E}$ ), 
interneurons ( 24 of 316 or $8 \%$ ), characterized by thin non-spiny dendrites, or cells that did not clearly fit into any of these categories (21 of 316 or $7 \%$ ).

The locations of morphologically categorized cells were plotted on coronal sections and these locations were transferred to a flattened representation of cortical areas as defined Wang and Burkhalter (2007; Figures 24 and 25). Recording sites were concentrated in the $\mathrm{LM} / \mathrm{LI}$ and $\mathrm{P} / \mathrm{POR}$, where terminals originating from the pulvinar are most densely distributed. V1 recordings were limited to cases in which no spread of virus into the adjacent dLGN could be detected, and TdTomato-labeled terminals were confined to layers I and Va within V1. Of the morphologically categorized cells, similar proportions of pyramidal cells (Figure 25A) and spiny stellate cells (Figure 25B) were found across cortical areas (of 152 filled cells in P/POR 28 or $18 \%$ were spiny stellate and 100 or $66 \%$ were pyramidal; of 67 filled cells in LM/LI, 10 or $15 \%$ were spiny stellate and 51 or $76 \%$ were pyramidal; of 44 filled cells in V1, 8 or $18 \%$ were spiny stellate and 28 or $63 \%$ were pyramidal).

\section{Cell types responsive to optogenetic activation of pulvinocortical terminals}

Optogenetic experiments were divided into 3 categories: 1) recordings from unidentified cells, 2) recordings specifically targeted to interneurons, and 3) recordings specifically targeted to cells with projections to the $\mathrm{SC}, \mathrm{V} 1$, or striatum and amygdala.

The majority (181 of 259 or $70 \%$ ) of unidentified cells responded to activation of pulvinar input (as defined above). Cells within the P/POR region were found to be the most responsive to optogenetic activation of pulvinar terminals (107 of 136 or 
$79 \%)$. In the LM/LI region 33 of 50 (66\%) were responsive to activation of pulvinar terminals, and in V1, 20 of 34 (59\%) were responsive.

Activation of pulvinar terminals with trains of light pulses $(1,2,5,10,20 \mathrm{~Hz})$ revealed an overall frequency-dependent depression of synaptic responses (Figure 22A-B, 23A-B). This is illustrated by plotting the ratio of the amplitude of the second EPSP divided by the first EPSP (paired pulse ratio) or the average of the amplitudes of the $2^{\text {nd }}-10^{\text {th }}$ EPSPs divided by the amplitude of the first EPSP (Train/ $1^{\text {st }}$ pulse ratio) (Figure 22F-G, 23F-G). In addition, membrane resistances were similar between all responsive pyramidal cells and spiny stellate cells (Figure 28A).

\section{Optogenetic activation of pulvinar terminals: effects on cortical interneurons}

To determine whether cortical interneurons receive input from the pulvinar, we used a transgenic mouse line in which GFP is expressed in a subset of GABAergic interneurons (GAD65-GFP). In this line, GFP is expressed in interneurons that contain the calcium-binding protein calretinin (Figure 26A-C) and/or other interneuron subtypes (López-Bendito et al., 2004), but GFP is not expressed in interneurons that contain parvalbumin (Figure 26D-F). Electron microscopic evaluation of GAD65-GFP-labeled profiles, in tissue additionally stained with a GABA antibody tagged with gold particles, revealed that GFP-labeled somata (Figure $26 \mathrm{G}$ ), and the majority of larger dendrites (Figure 26H) contained GABA (gold particle density overlying GFP-labeled profiles larger than $0.35 \mu \mathrm{m}^{2}$ was $52.60 \pm$ 30.33 gold particles $/ \mu \mathrm{m}^{2}$ ). However, GFP-labeled profiles within the size range of dendrites postsynaptic to pulvinar terminals (Figure 26I) were often devoid of any 
overlying gold particles (no gold particles were found overlying 70 of 136 , or $52 \%$, of GFP-labeled profiles less than $0.35 \mu \mathrm{m}^{2}$ ). This indicates that GABA is not consistently detectable in smaller dendrites using standard postembedding immunohistochemical techniques. For this reason, we were unable to determine whether pulvinar-cortex terminals contact GABAergic interneurons using anatomical techniques alone.

Virus injections were placed in the pulvinar of GAD65-GFP mice and in subsequent slice experiments whole cell recordings were obtained from GFP-labeled neurons (Figure 27A-B, $n=34$, locations plotted in Figure 25C), and surrounding pulvinar terminals were activated with blue light. The input resistance of responsive GFP-labeled interneurons $(180.5 \pm 85.34 \mathrm{~m} \Omega)$ was significantly greater than that of responsive pyramidal cells $(78.2 \pm 36.63 \mathrm{~m} \Omega)$ and responsive spiny stellate cells $(96.8 \pm 72.93 \mathrm{~m} \Omega$; Figure 28A), and the morphology of successfully-recovered biocytin-filled GFP-labeled cells $(n=24)$ was consistent with previous descriptions of interneurons (i.e. smooth dendrites; Figure 27C-E). We found that the majority $(25 / 34$ or $74 \%$; Figure $27 \mathrm{H}$ ) of GFP-labeled cells responded to optogenetic activation of pulvinar terminals ( 1 or $10 \mathrm{~ms}$ duration, $10-112 \mathrm{~mW} / \mathrm{mm}^{2}$ blue light pulses through the microscope objective) with large amplitude EPSPs or EPSCs (1.064-26.22 mV; 35.72-699.7 pA), with short $(<4.6 \mathrm{~ms})$, fixed latencies. Example responses are illustrated in Figure 25A-B. When pulvinar terminals were stimulated with train of light pulses, the interneuron responses generally exhibited frequency-dependent depression (Figure 27A-B). However, paired pulse ratio plots (Figure 27F) and 
train $/ 1^{\text {st }}$ pulse ratio plots (Figure $27 \mathrm{G}$ ) demonstrate the variability observed between neurons. Further demonstrating the involvement of interneurons in pulvinar-cortex circuits, application of a $\mathrm{GABA}_{\mathrm{A}}$ receptor antagonist $(\mathrm{SR} 95531,20 \mu \mathrm{M}, \mathrm{n}=6)$ during photostimulation of pulvinocortical terminals increased the EPSPs up to 4 fold (Figure 28B, $n=2$ ), or induced spikes (Figure 28C, $n=4$ ).

\section{Optogenetic activation of pulvinar terminals: effects on cortical output neurons}

To determine whether terminals that originate from the pulvinar can directly influence the activity of cortical projection neurons, we paired pulvinar virus injections with CTB-488 or HSV injections in the ipsilateral SC, V1, or striatum/amygdala. The CTB or HSV injections labeled corticotectal, corticocortical or corticostriatal/amygdala cells, which we then targeted for recording using epifluorescent visualization of cortex slices maintained in vitro. Recordings were limited to cortical regions that contained overlapping distributions of CTB- or HSVlabeled cells and TdTomato-labeled thalamocortical terminals originating from the pulvinar (Figure 29A-C), and in which responses could be evoked in non-CTBlabeled cells within the same experiment. CTB or virus-induced labeling of recorded cells was confirmed by video recordings of the patched cells and/or subsequent imaging of CTB-488 or YFP within recovered biocytin-filled cells using a confocal microscope (Figure 29Ai-iii, Bi-iv and Ci-iv).

The distribution of corticotectal cells was limited to layer V in V1 and the LES. Corticocortical cells projecting to V1 were distributed in layers V and VI of the LES (Figure 29Bi). Corticostriatal/amygdala cells were confined to layer V within 
V1, but their distribution expanded in the more ventral and caudal regions of the LES; in the LM and LI, most corticostriatal/amygdala cells were confined to layer V, but within P and POR corticostriatal/amygdala cells were distributed throughout most layers (Figure 29Ci and Ciii). The increase in the density of corticostriatal/amygdala cells in ventral/caudal cortical areas mimicked the increase in the density of pulvinar projections in ventral/caudal cortical areas.

Whole cell recordings were obtained from a total of 120 cells labeled by retrograde transport (31 corticotectal, 34 corticocortical and 68 corticostriatal/amygdala cells; Figure 29F). Of these 3 cell groups, corticostriatal/amygdala cells were by far the most responsive group; 52 of 68 or $76 \%$ of corticostriatal/amygdala cells responded to activation of surrounding pulvinar terminals with short latency $(\leq 5.7 \mathrm{~ms})$. Using these same criteria, 18 of 34 corticocortical cells $(55 \%)$, and 2 of 31 corticotectal cells $(6 \%)$ were categorized as responsive. Comparison of the maximum EPSP or EPSC amplitudes elicited in these cell groups via activation of pulvinar terminals also revealed significant differences, with corticocortical cells exhibiting the most robust responses (Figure 29D-E).

Activation of pulvinar input to projection cells with trains of light pulses revealed a frequency-dependent depression of synaptic responses (Figure 29G and H). Furthermore, in the presence of TTX and 4AP, large amplitude responses could be elicited (Figure 29I, corticostriatal/amygdala $n=10$, corticocortical $n=5$ ) which were abolished by subsequent application of the AMPA and NMDA receptor antagonists (Figure 29I, corticostriatal/amygdala, $n=4$, corticocortical $n=1$ ). The locations of all 
recovered corticostriatal/amygdala cells were plotted (Figure 25D). Most responsive cells were concentrated in the P/POR region. The majority of corticostriatal/amygdala cells were pyramidal cells, but four were categorized as spiny stellate cells.

\section{Discussion}

We found that the mouse pulvinar projects densely to interconnected regions of the LES, striatum and amygdala. The laminar distribution and ultrastructure of pulvinocortical terminals in the LES was found to be nearly identical to that of geniculocortical terminals in V1, and optogenetic activation of pulvinocortical terminals strongly depolarized pyramidal cells, spiny stellate cells and interneurons in layers IV and V of the LES. Furthermore, recordings targeted to specific projection neuron subtypes within layer $\mathrm{V}$ revealed that the pulvinar strongly affects the activity of corticostriatal, corticoamygdala and corticocortical cells in the LES via direct and indirect synaptic contacts. This information coupled with the input and output organization of the pulvinar nucleus, suggest that the pulvinar is a pivotal component of circuits used for the visual guidance of movement.

\section{Morphology of thalamocortical terminals and their postsynaptic targets}

We compared the ultrastructure of thalamocortical terminals derived from either the dLGN or pulvinar nucleus of the mouse. We found that the sizes of pulvinocortical terminals in the extrastriate cortex were not significantly different than the sizes of geniculocortical terminals in layer IV of V1. Comparison of terminal types in the Galago demonstrated that pulvinocortical terminals are smaller than layer

IV geniculocortical terminals originating from the magnocelllular layers of the dLGN, 
but not significantly different from geniculocortical terminals originating from parvocellular layers (Marion et al., 2013). However in the tree shrew, pulvinocortical terminals are smaller than all layer IV geniculocortical terminals (Chomsung et al., 2010; Familtsev et al., 2016), and other features, such as dendritic protrusions (Erisir and Dreusicke, 2005) are found within geniculocortical terminals, but not pulvinocortical terminals. The differences between these terminals types across species may be related to the distribution of spiny stellate cells which are densely packed in V1 layer IV of non-rodent species (Fitzpatrick, 1996; Callaway and Borrell, 2011; da Costa and Martin, 2011; Muly and Fitzpatrick 1992).

We found that terminals that originate from the mouse pulvinar nucleus contact spines and other small dendritic profiles, similar to pulvinocortical terminals in the tree shrew (Chomsung et al., 2010). In the tree shrew, it was concluded that pulvinocortical terminals contact pyramidal or spiny stellate cells based on the absence of detectable GABA within postsynaptic profiles. This is consistent with our identification of pyramidal and spiny stellate cells as postsynaptic targets or pulvinocortical terminals in the mouse. However, our optogenetic experiments in mice also identified interneurons as targets of pulvinocortical terminals, which prompted us to critically evaluate the ability to detect profiles that originate from cortical interneurons using postembedding immunocytochemical staining for GABA. By comparing GAD65-GFP-labeled elements with GABA-stained elements, we conclude that, in contrast to thalamic interneurons which release GABA from dendritic terminals (Govindaiah and Cox, 2004; Bickford et al., 2010), cortical 
interneurons do not accumulate detectable levels of GABA within small diameter dendrites, presumably because they release GABA only from axon terminals (Nahmani and Turrigiano, 2014).

Previous studies of the mouse somatosensory cortex have demonstrated that spiny stellate cells are a major target of thalamocortical terminals which form asymmetric axospinous synapses (White and Rock, 1979; Benshalom and White, 1986). However, our study provides the first evidence in mouse that spiny stellate cells are also located in both V1 and the LES. In the ferret striate cortex, spiny stellate cells develop from pyramidal neurons by a visual experience-dependent process of apical dendrite pruning (Callaway and Borrell, 2011). It is possible that our detection of spiny stellate cells was facilitated by the fact that we used mice at older ages than previous studies of mouse V1 (e.g Kloc and Maffei, 2014). However, spiny stellate cells in the mouse somatosensory cortex exhibit their mature morphology by P5 (Callaway and Borrell, 2011). Therefore, it is most likely that detection of spiny cells was simply the result of our large sample size (316 neurons with complete filling); in all visual cortical areas, spiny stellate cells made up only $15-18 \%$ of sampled neurons.

\section{Projection neurons of the mouse visual cortex}

Recently, 3 genetically-distinct types of layer 5 projection neurons were identified in V1 (Kim et al., 2015): 1) corticocortical (CC) neurons that project to the striatum and other cortical areas, 2) corticosubcortical (CS) neurons that project to the superior colliculus, thalamus, brainstem and striatum, and 3) corticocorticalnonstriatal (CC-NS) neurons that project locally within the cortex, but not to striatum. 
We find that pulvinocortical projections primarily target cortical cells in LES that project to the striatum/amygdala $(76 \%)$ or to $\mathrm{V} 1(55 \%)$. Thus it is possible that pulvinocortical projections target a single class of cells that have branching projections to the striatum and V1. However, it is difficult to directly compare our results to those of Kim et al (2015). Monosynaptic circuit tracing from the 3 defined cells in V1 did not reveal a differential labeling of pulvinar neurons, but this experimental method does not reveal strength of synaptic input. Most importantly, we focused most or our recordings in extrastriate areas of the cortex. Since the genetic labeling patterns that classify cell types in V1 likely differ in extrastriate areas of cortex, it remains to be determined whether pulvinocortical terminals preferentially target a single genetically-defined cell type. Our study of the morphology and projection patterns of neurons targeted by the pulvinar nucleus lays the groundwork for further thalamocortical circuit analysis that includes genetically-defined cells types.

\section{The pulvinar nucleus and anesthesia}

We found that pulvinocortical projections can strongly impact the activity of neurons in the extrastriate cortex, supporting the view that visually-evoked activity in extrastriate areas may be primarily relayed via the pulvinar nucleus. However, this view is at odds with the conventional hierarchical view of the cortex in which the transfer of visual information to extrastriate cortical areas is considered to be primarily relayed from V1 via corticocortical connections (Van Essen, 2005). Anesthesia could at least partially account for these disparate views. Recordings in 
anesthetized animals support a hierarchical view in that silencing V1 activity results in a profound depression of responses in extrastriate areas (Girard et al., 1992; Kaas and Krubitzer, 1992; Azzopardi et al., 2003). However, in anesthetized mice, spontaneous activity in the pulvinar nucleus is significantly lower than that recorded in the dLGN (Roth et al., 2016), and the proportion of pulvinar neurons that respond to simple visual stimuli is approximately half that of dLGN neurons (Allen et al., 2016). Even in awake but inactive primates, the spontaneous activity of pulvinar neurons is less than half that of dLGN neurons (Ramcharan et al., 2005). Moreover, recordings in awake running mice have revealed that visual activity in V1 is affected by movement of the animal even though dLGN activity is unaffected (Niell and Stryker, 2010). These various pieces of evidence suggest that the full influence of pulvinocortical projections can only be assessed in awake, active animals.

\section{Is the pulvinar nucleus a hub for visually-triggered action selection?}

The mouse pulvinar nucleus receives dense input from wide-field-vertical (WFV) cells in the superior colliculus (Zhou et al., 2017).The large dendritic fields of WFV cells have been described as motion detectors (Major et al., 2000); they respond preferentially to small objects moving across the visual field in any direction (Gale and Murphy, 2014, 2016). Our results indicate that in addition to the direct projections of the pulvinar nucleus to the striatum and amygdala (Day-Brown et al., 2010), pulvinar projections to the cortex preferentially target corticostriatal and corticoamygdala cells. These input and output connections of the pulvinar nucleus suggest that it can be viewed as a hub involved in the initiation or alteration of the 
appropriate actions in response to the detection of visual movement. Recent optogenetic studies support this idea. Activation of a SC-pulvinar-amygdala pathway has been shown to elicit freezing responses, whereas inactivation of this pathway inhibits innate freezing response elicited by overhead looming stimuli (Wei et al., 2015). Given the large repertoire of behaviors that can now be quantified using mice (Yilmaz and Meister, 2013; De Franceschi et al., 2016; Hoy et al., 2016), future optogenetic and/or chemogenetic manipulations may help to unravel the specific contributions of the pulvinocortical circuits to the initiation of apt behavioral responses.

\section{The pulvinar and visual neglect}

Visual neglect syndrome causes human patients to ignore the visual field contralateral to lesions of the pulvinar nucleus, striatum, and/or extrastriate regions surrounding the temporal-parietal junction (Karnath et al., 2002; Smith et al., 2013). In particular, these patients make few eye or body movements directed toward the neglected space. Our results suggest that these particular lesion sites may disrupt a network of pulvinar connections that link the cortex and striatum. While the mouse of course is not an adequate model for all aspects of this human condition, it can inform and guide further studies of the pulvinar nucleus that may lead to therapeutic interventions. 
Figure 20. Laminar distribution and ultrastructure of thalamocortical terminals originating from the dLGN and pulvinar

A. In V1, thalamocortical terminals originating from the dLGN primarily innervate layers I and IV, and corticogeniculate cells are concentrated in layer VI. B. In V1, sparser thalamocortical terminals originating from the pulvinar innervate layers I and Va. C. In LM/LI, dense terminals originating from the pulvinar innervate layers I and IV. Corticothalamic cells that project to the pulvinar are seated in layer VI. D-F. Examples of thalamocortical terminal ultrastructure: D. dLGN terminal in V1 layer I. E. pulvinar terminal in LM/LI. F. pulvinar terminal in P/POR. D-F. Labeled terminals are dark gray, postsynaptic dendrites are light blue, and arrows indicate synapses. GH. Comparisons of the size of presynaptic thalamocortical terminals and their postsynaptic dendrites. The horizontal bar within each box indicates the mean terminal/dendrite size, the box boundaries indicate the lower and upper quartiles $(25 \%$ and $75 \%$, respectively), and the vertical lines (whiskers) indicate the full range of terminal/dendrite sizes. Significant differences in size indicated by $*(P<0.05,1$ way ANOVA, Post-hoc Tukey's Multiple Comparison Test) or ** $(\mathrm{P}<0.01)$. Scale bar: A-C $50 \mu \mathrm{m}, \mathrm{D}-\mathrm{F} 600 \mathrm{~nm}$. 


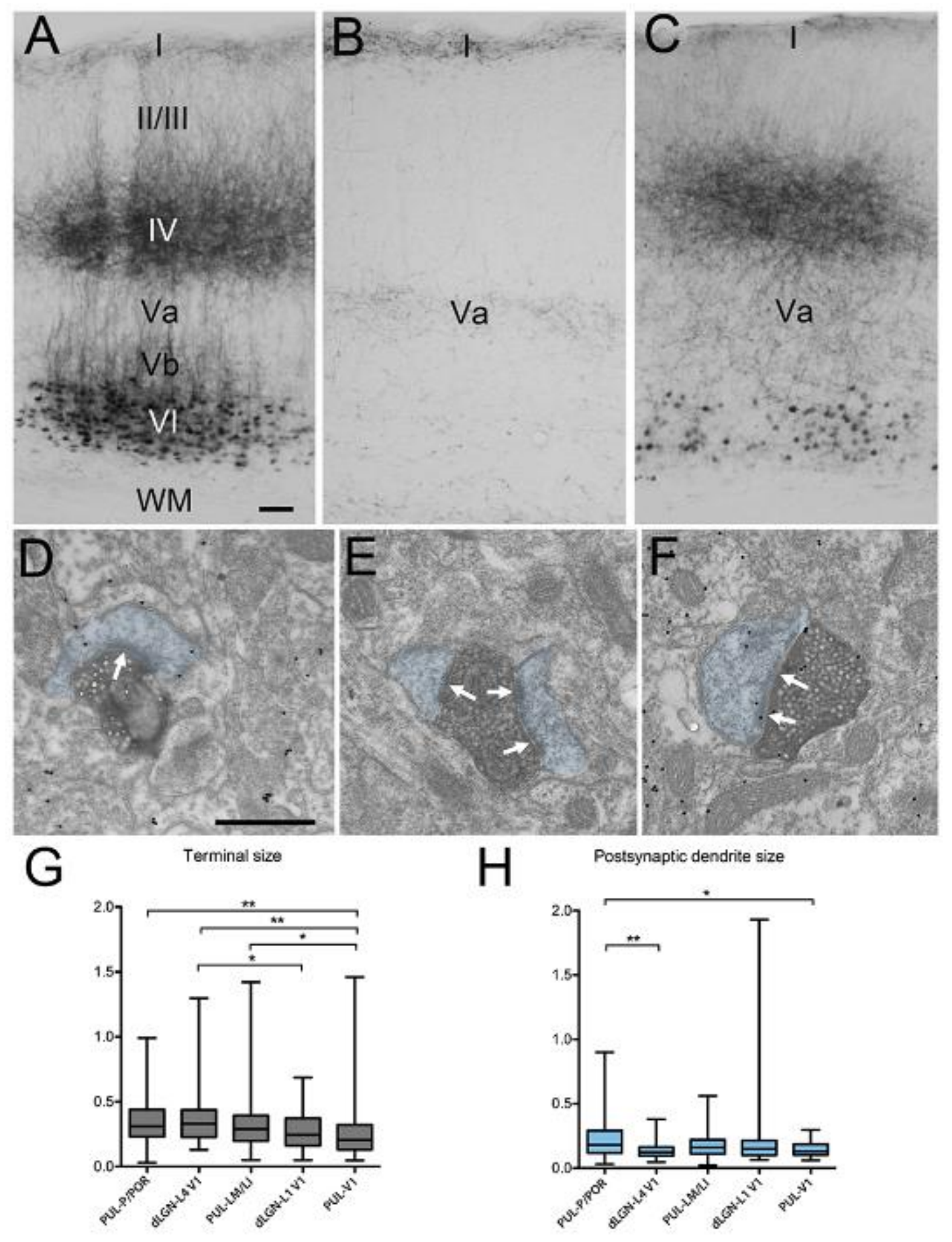


Figure 21. Optogenetic activation of thalamocortical terminals that originate

\section{from the pulvinar}

A. Schematic illustrates the experimental protocol. Virus injections in the pulvinar induced expression of ChIEF and Tdtomato in pulvinar-cortex terminals (red). Ten days later, slices of the cortex were prepared for in vitro whole cell patch recordings in the area of labeled terminals. Biocytin (green) was included in the pipettes to fill cells while recording their responses to blue light pulses. B. Injection site in the pulvinar. C. Induced Tdtomato expression in pulvinar-cortex terminals in V1 and LES. D-E. Higher magnification of pulvinar terminals in V1 and LES. F. pulvinarLES responses were light-intensity dependent and could be elicited in the presence of $1 \mu \mathrm{M}$ tetrodotoxin (TTX) when paired with $1 \mathrm{mM} 4$-aminopyridine (4AP), and were blocked by the application of $80 \mu \mathrm{M}$ 6,7-dinitroquinoxaline-2,3-dione (DNQX) and $10 \mu \mathrm{M}$ DL-2-amino-5-phosphono-valeric acid (APV), indicating that glutamate release from pulvinar terminals activates AMPA and NMDA receptors. G. Pyramidal cells (green) that responded to light activation of surrounding pulvinar-cortex terminals (red). Scale bars: B-C $250 \mu \mathrm{m}$, D-E $100 \mu \mathrm{m}, \mathrm{G} 50 \mu \mathrm{m}$. dLGN, dorsal lateral geniculate nucleus, PM, posteromedial cortex, PT, pretectum, vLGN, ventral lateral geniculate nucleus. 


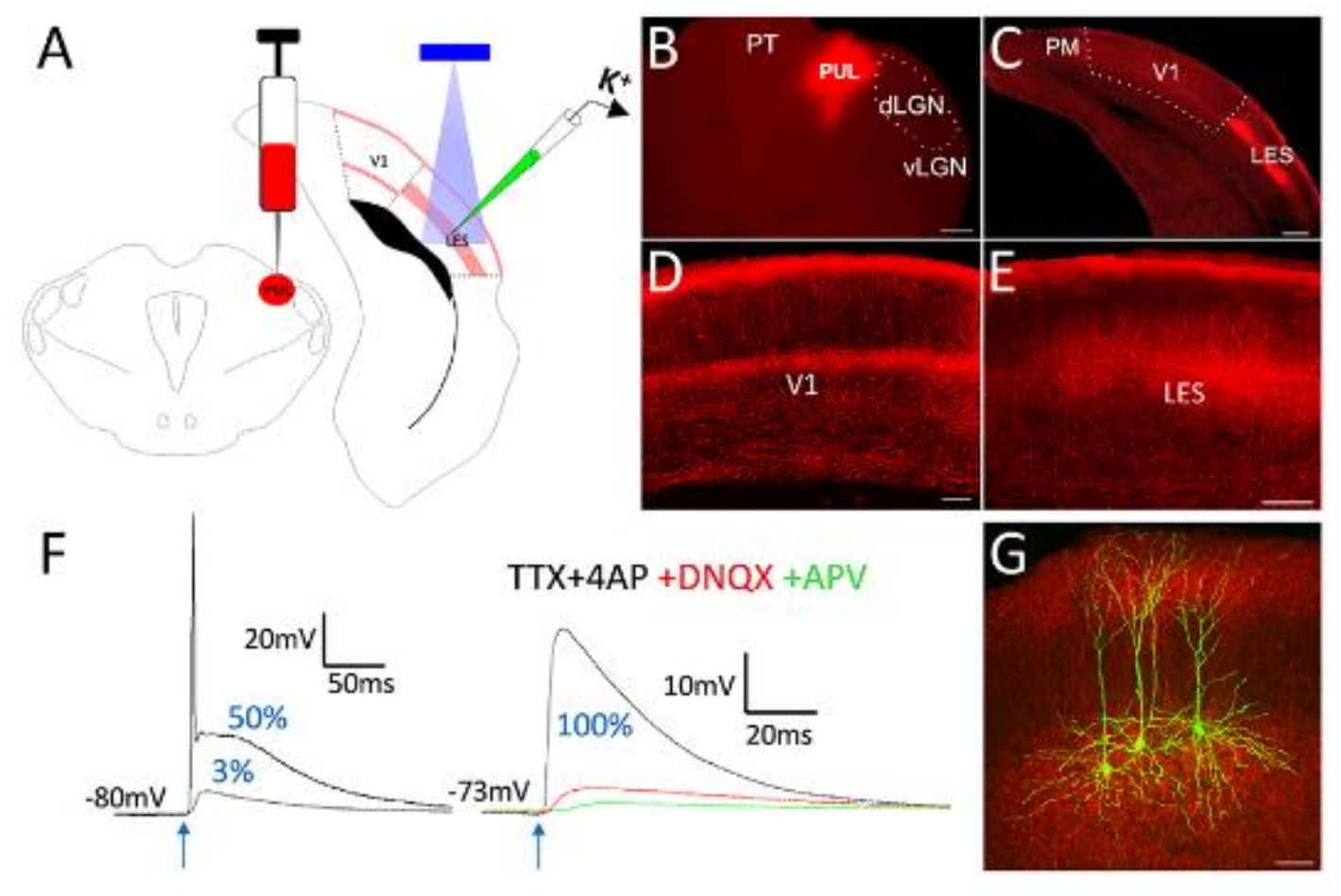




\section{Figure 22. Pulvinar activation of pyramidal cells}

A. Responses of a pyramidal cell to photoactivation of pulvinar terminals. EPSPs (A) and EPSCs (B) were induced by a train of 10 light pulses (10ms duration, indicated by the blue bars) at 1HZ, 2HZ, 5HZ, 10HZ and 20HZ. C-E. Examples of biocytin-filled pyramidal cells that were recovered after recording. F-G. Plots of the ratio of the amplitude of the second EPSP divided by the first EPSP (paired pulse ratio, F), or the average of the amplitudes of the $2^{\text {nd }}-10^{\text {th }}$ EPSPs divided by the amplitude of the first EPSP (Train $/ 1^{\text {st }}$ pulse ratio, G) for 48 pyramidal cells. H. Of 174 recorded pyramidal cells, $118(68 \%)$ responded to photoactivation of pulvinar terminals. Scale bar: C-E $50 \mu \mathrm{m}$. 

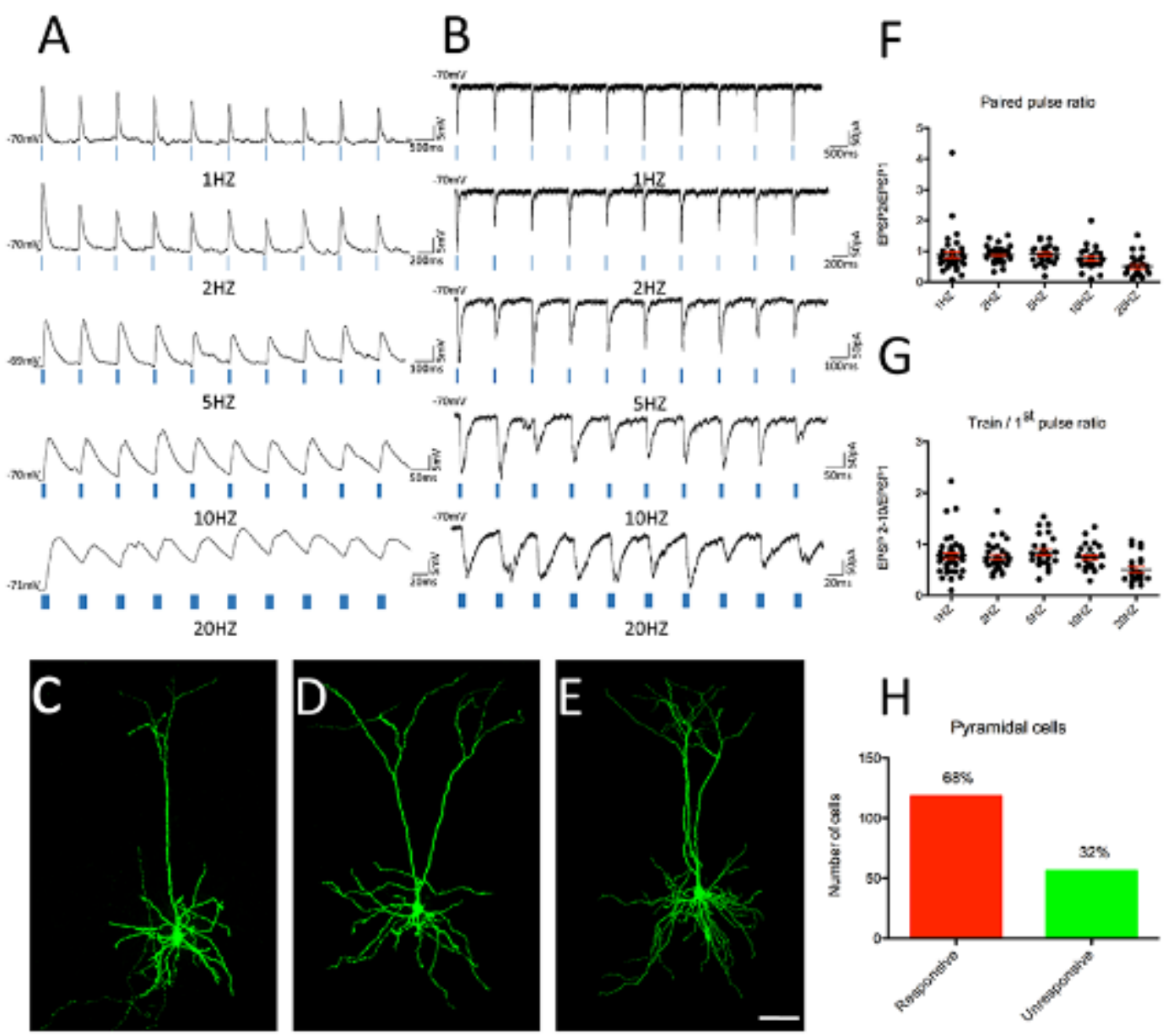
Figure 23. Pulvinar activation of spiny stellate cells

A, B. Responses of spiny stellate cells to photoactivation of pulvinar terminals. EPSPs (A) or EPSCs (B) were induced by a train of 10 light pulses (1-10 ms duration, indicated by the blue bars) at $1 \mathrm{HZ}, 2 \mathrm{HZ}, 5 \mathrm{HZ}, 10 \mathrm{HZ}$ and 20HZ. C-E. Examples of biocytin-filled spiny stellate cells that were recovered after recording. F-G. Plots of the ratio of the amplitude of the second EPSP divided by the first EPSP (paired pulse ratio, F), or the average of the amplitudes of the $2^{\text {nd }}-10^{\text {th }}$ EPSPs divided by the amplitude of the first EPSP (Train $/ 1^{\text {st }}$ pulse ratio, G) for 18 spiny stellate cells. H. Of $46 \mathrm{recorded} /$ recovered spiny stellate cells, $30(65 \%)$ responded to photoactivation of pulvinar terminals. Scale bar: C-E $20 \mu \mathrm{m}$. 

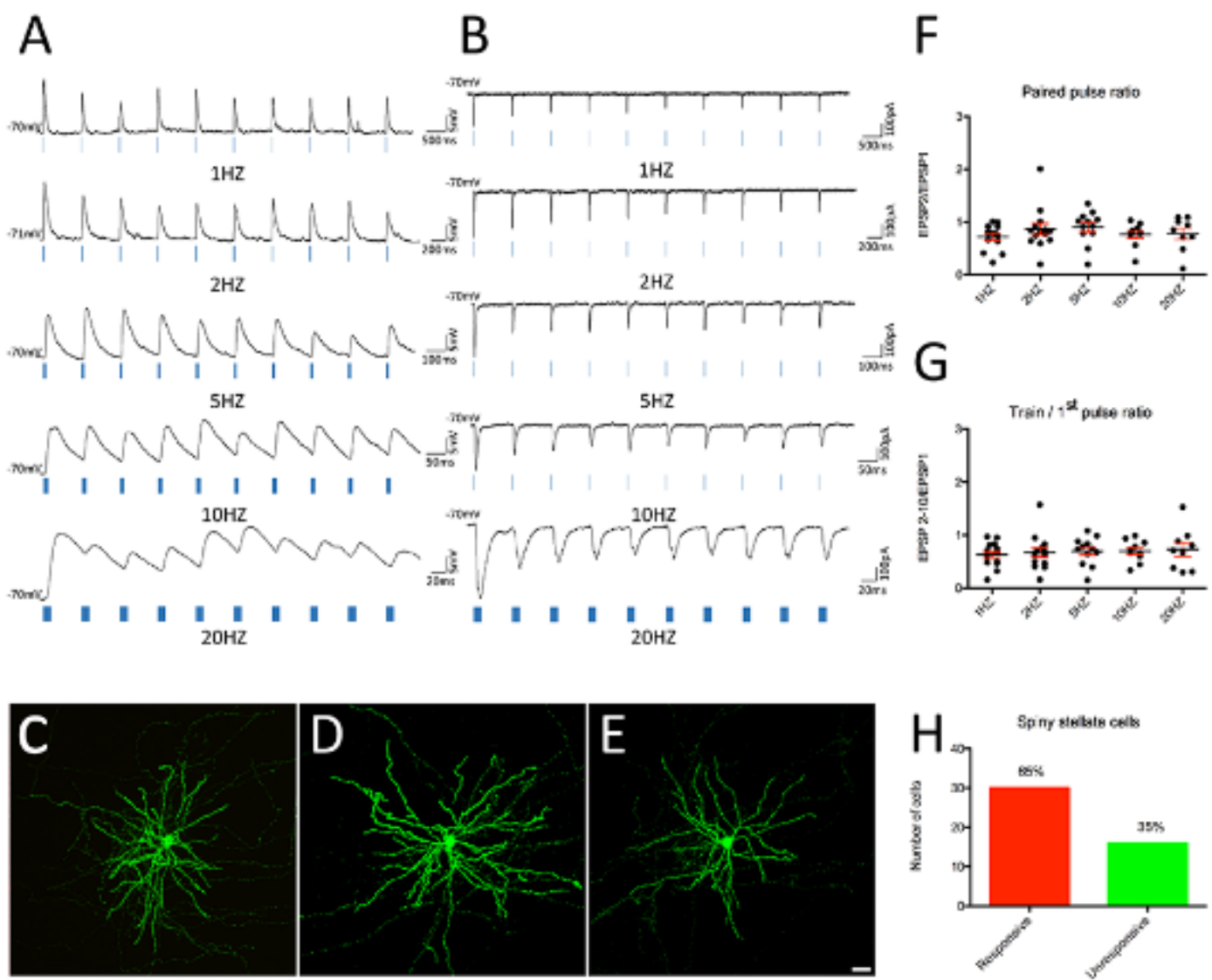


\section{Figure 24. Creation of flattened map from coronal sections}

Virus injections in the pulvinar label projections to $\mathrm{V} 1$ and extrastriate areas. A) A one in two series of sections through the caudal regions of cortex was processed to reveal the projections of the pulvinar with a diaminobenzidine reaction. B) The approximate location of each section is indicated in the flattened cortex map (modified from Wang and Burkhalter, 2007). A, anterior, AL, anterior lateral, AUD, auditory cortex, D, dorsal, L, lateral, LI, laterointermediate, LM, lateromedial, P, posterior, PM, posterior medial, POR, postrhinal, TeA, temporal association area, V, ventral. 


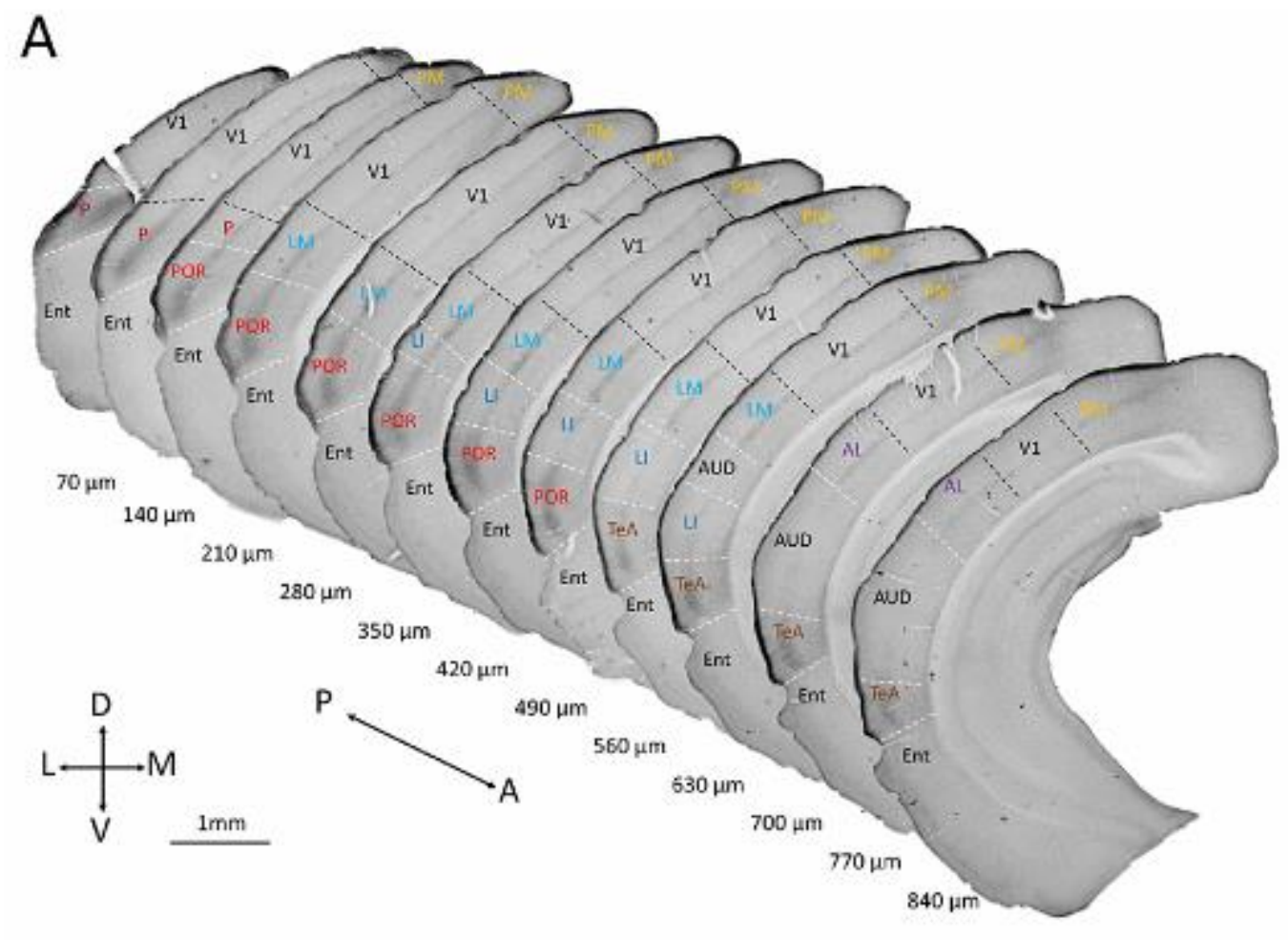

B

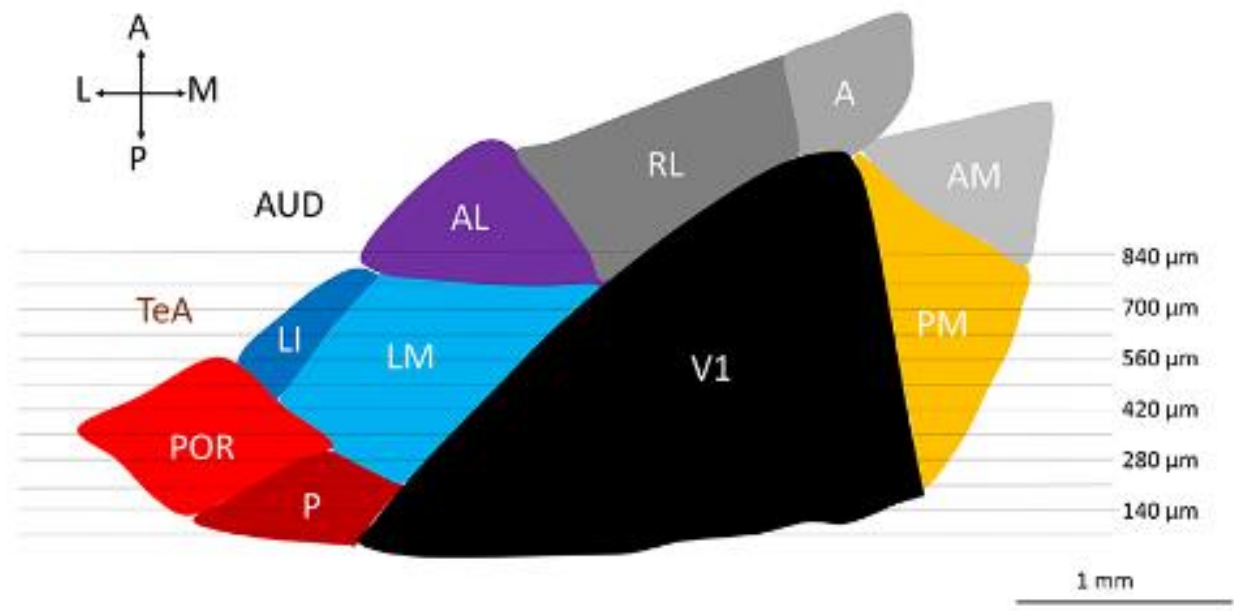


Figure 25. Distribution of cells that activated by the pulvinar

A-D. The distribution of recovered cells converted from coronal sections to a flattened cortex map based on Wang and Burkhalter (2007, J Comp Neurol 502(3):339-57). Regions of interest are indicated with black lines. Dotted lines indicate coronal section spacing. Cells that responded to pulvinar innervation are indicated with red dots, unresponsive cells are indicated with green dots, and cells filled with biocytin but not tested for pulvinar input are indicated with black dots. A, anterior, anterior area, $\mathrm{AL}$, anterolateral area, $\mathrm{AM}$, anteromedial area, $\mathrm{AUD}$, auditory cortex, D, dorsal, L, lateral, LI, laterointermediate area, LM, lateromedial area, P, posterior, area, PM, posteromedial area, POR, postrhinal area, RL, rostrolateral area, TeA, temporal association area, V, ventral. Scale bar: $1 \mathrm{~mm}$. 


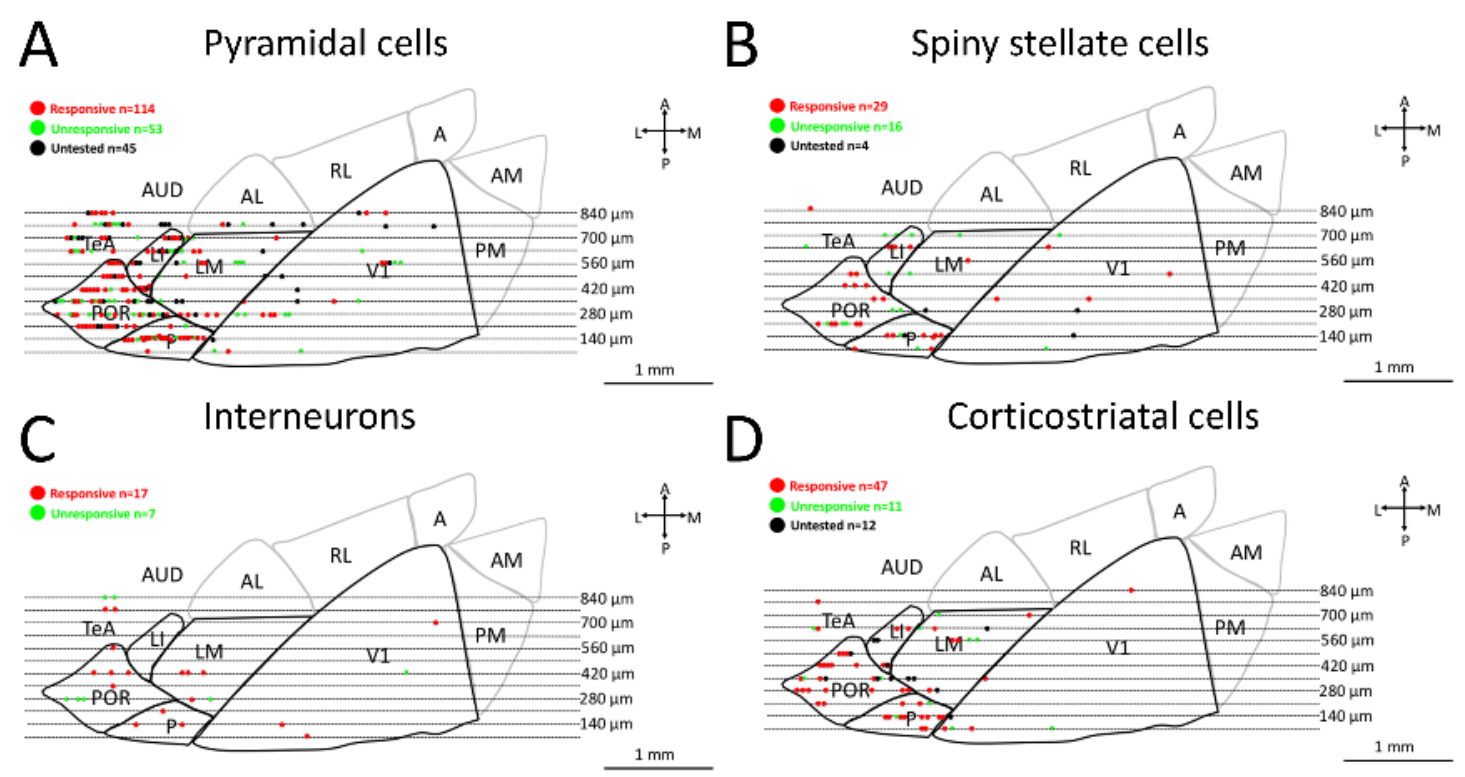




\section{Figure 26. Characterization of GAD65-GFP cells in V1}

V1 sections that contain GFP-labeled interneurons (green, A, C, D, F) were stained with antibodies against calretinin (purple B, C), parvalbumin (purple E, F), or GFP (dark reaction product, G-I) and GABA (gold particles, G-I). GFP often co-localized with calretinin (white cells in $\mathrm{C}$ indicated by arrows) but rarely with parvalbumin (F). GFP-labeled somata $(\mathrm{G})$ and larger dendrites $(\mathrm{H})$ contained detectable levels of GABA (high density of gold particles), but GABA was often undetectable in smaller GFP-labeled profiles (I). Scale in $\mathrm{C}=20 \mathrm{um}$ and applies to A-F. Scale in $\mathrm{I}=600 \mathrm{~nm}$ and also applies to $\mathrm{G}$ and $\mathrm{H}$. 


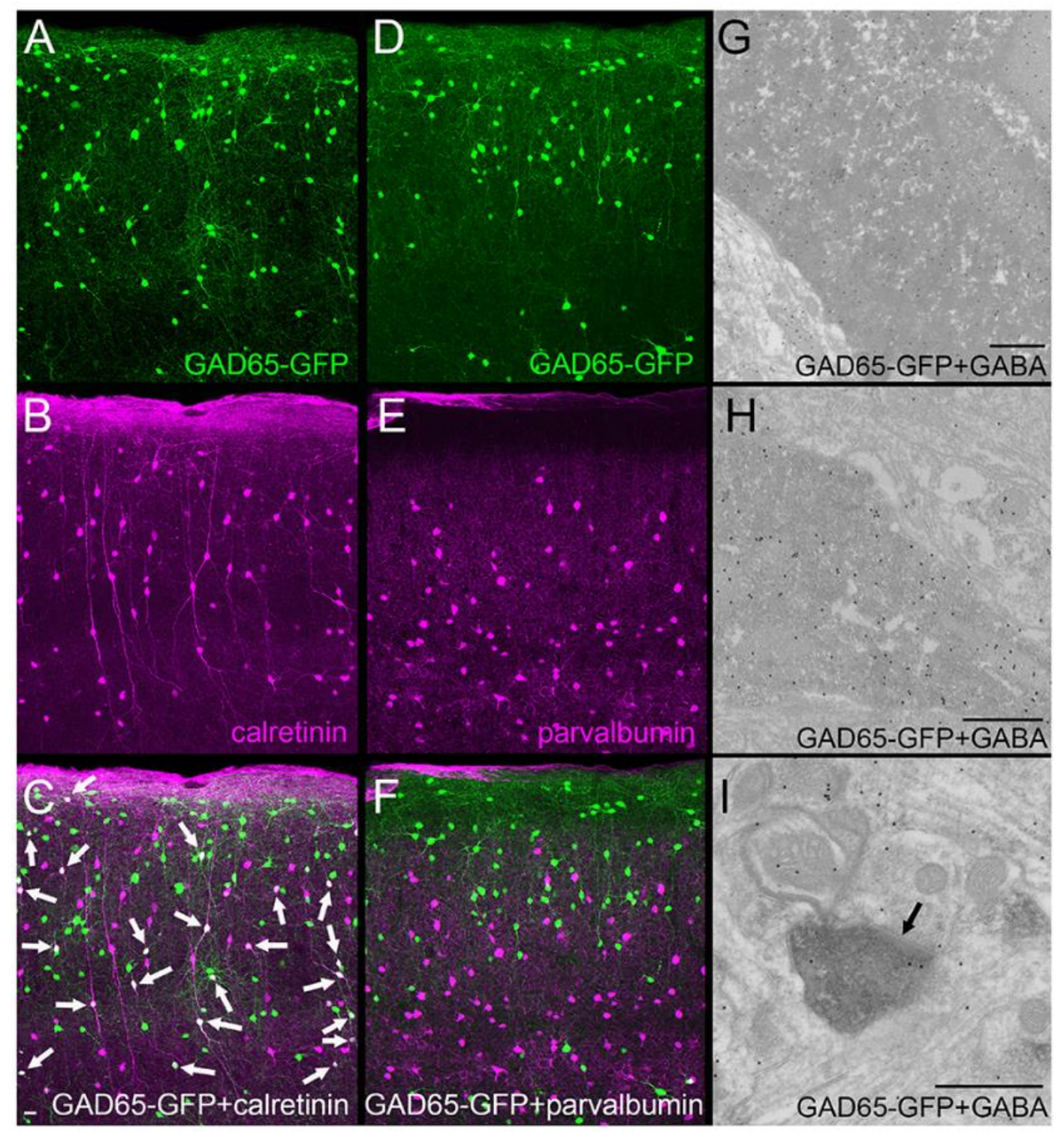




\section{Figure 27. Pulvinar activation of GAD65 interneurons}

A. In GAD65-GFP transgenic mice, responses of an interneuron to photoactivation of pulvinar terminals. EPSPs (A) and EPSCs (B) were induced by a train of 10 light pulses (10ms duration, indicated by the blue bars) at $1 \mathrm{HZ}, 2 \mathrm{HZ}, 5 \mathrm{HZ}, 10 \mathrm{HZ}$ and 20HZ. C-E. e Examples of GFP-labeled (green) biocytin-filled interneurons (red) that were recovered after recording. F-G. Plots of the ratio of the amplitude of the second EPSP divided by the first EPSP (paired pulse ratio, F), or the average of the amplitudes of the $2^{\text {nd }}-10^{\text {th }}$ EPSPs divided by the amplitude of the first EPSP (Train/ $1^{\text {st }}$ pulse ratio, G) for 25 interneurons. H. Of 34 recorded interneurons, 25 cells (74\%) responded to photoactivation of pulvinar terminals. Scale bar: C-E $50 \mu \mathrm{m}$. 
A

B

F

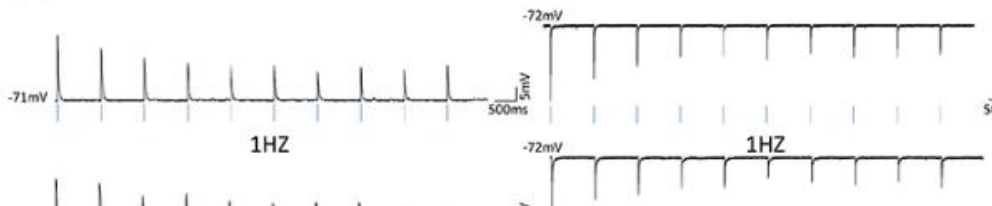

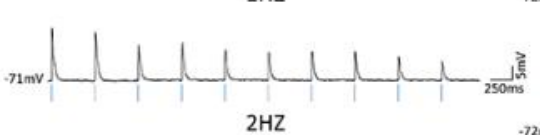

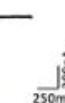

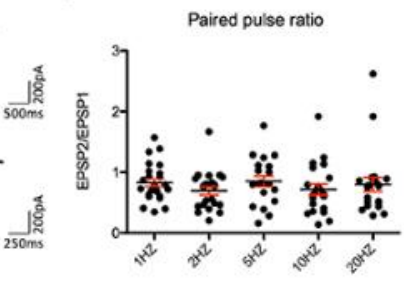

-70mva

$2 \mathrm{HZ}$

售 $\mathrm{G}$

(70mv

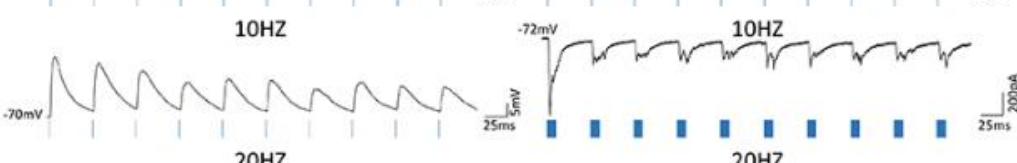

Train $/ 1^{\text {st }}$ pulse ratio

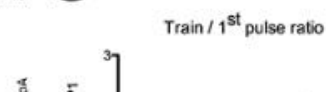

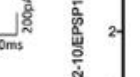

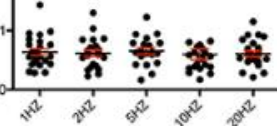
$20 \mathrm{HZ}$
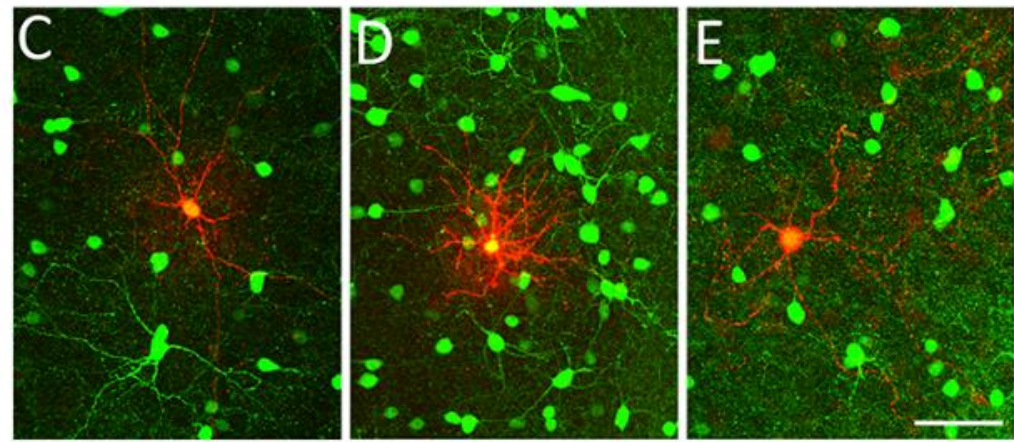

$\mathrm{H}$

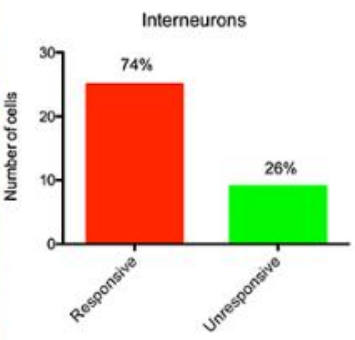




\section{Figure 28. Interneuron properties}

A. The membrane resistance of interneurons is statistically bigger than both of spiny

stellate cells and pyramidal cells. ${ }^{* * *} \mathrm{P}=0.0003, * * * * \mathrm{P}<0.0001$, one-way ANOVA test (Kruskal-Wallis test with Dunn's multiple comparison post-hoc test). Mean $\pm \mathrm{SD}$ :

interneurons, $157.8 \pm 91.06, \mathrm{n}=34$; spiny stellate cells, $72.95 \pm 25.33, \mathrm{n}=29$;

pyramidal cells, $74.6 \pm 37.07, n=81)$. B. 6 interneurons have been tested with GABAA $_{A}$ blocker SR95531, the amplitude of EPSP of all cells are increased after application of $\mathrm{GABA}_{\mathrm{A}}$ blocker, 4 of 6 are firing spikes, example is shown in $\mathrm{C}$. 
A

Membrane resistance
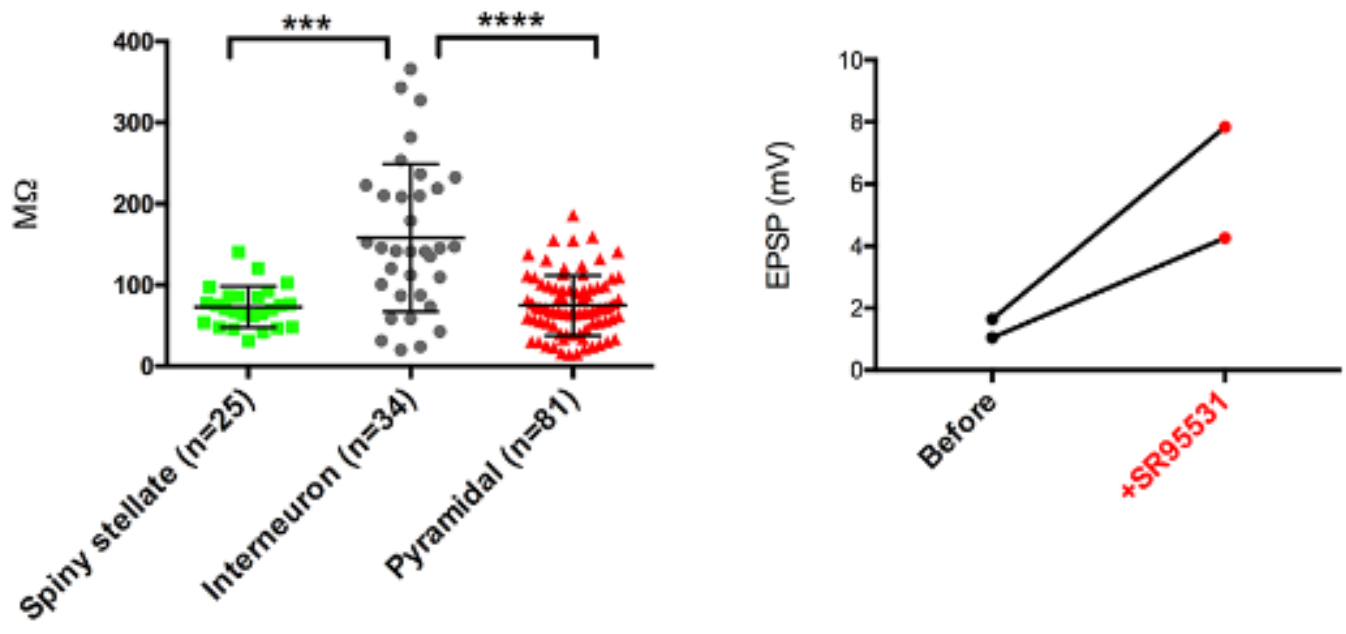

C

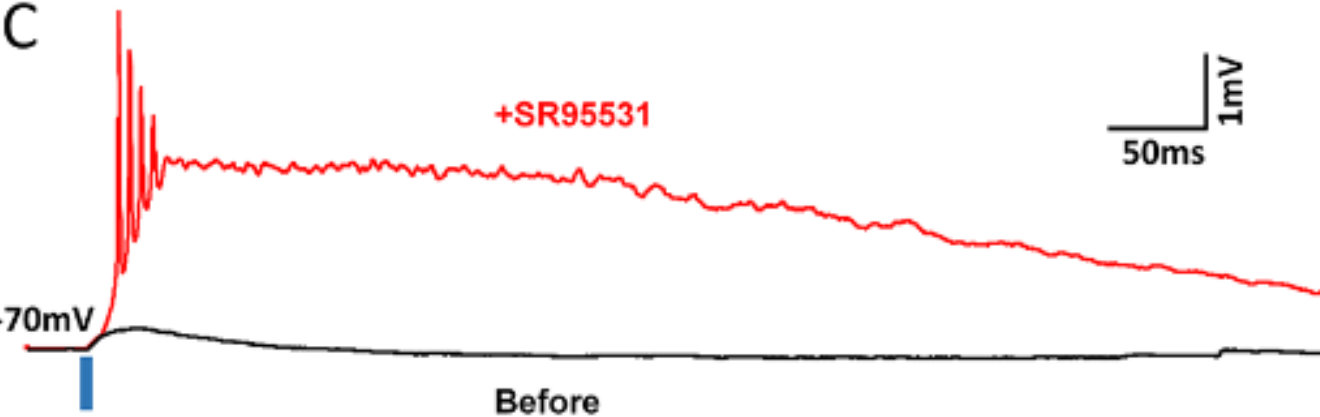




\section{Figure 29. Pulvinar activation of extrinsic projection cells}

A-C. Schematics illustrate the experimental protocol. Virus injections in the pulvinar were paired with CTB-488 injections into superior colliculus, V1 or striatum to label corticotectal, corticocortical or corticostriatal cells for targeted recordings. Ai.

Example of filled corticotectal cells. Aii-Aiii. Higher magnification of the CTB within the somata of the two cells biocytin-filled cells in Ai. Bi. Example of filled corticocortical cells. Bii-Biv. Higher magnification of the three cells in $\mathrm{Bi}$. $\mathrm{Ci}$ and Ciii. Example of filled corticostriatal cells. Cii and Civ. Higher magnification of cells in $\mathrm{Ci}$, Ciii, respectively. D-E. Maximum EPSP/EPSC amplitude for responsive corticotectal, corticocortical and corticostriatum cells. F. Of 31 tested corticotectal cells, 2 cells $(6 \%)$ were responsive to pulvinar innervation. Of 33 tested coticocortical cells, 18 cells (55\%) were responsive to pulvinar innervation. Of 68 corticostriatal cells, 52 cells $(76 \%)$ were responsive to pulvinar innervation. G-H comparison of the paired-pulse ratio and Train $/ 1^{\text {st }}$ pulse ratio for 17 corticocortical cells, 44 corticostriatal cells and 25 interneurons. I. Responses of a corticostriatal cell to photoactivation of pulvinar terminals in the presence of TTX and 4AP. This response was blocked by the sequential application of APV and CNQX. Scale bar: Ai-Ci and Ciii $50 \mu \mathrm{m}$; Aii-Aiii, Bii-Biv, Cii and Civ $10 \mu \mathrm{m}$ 


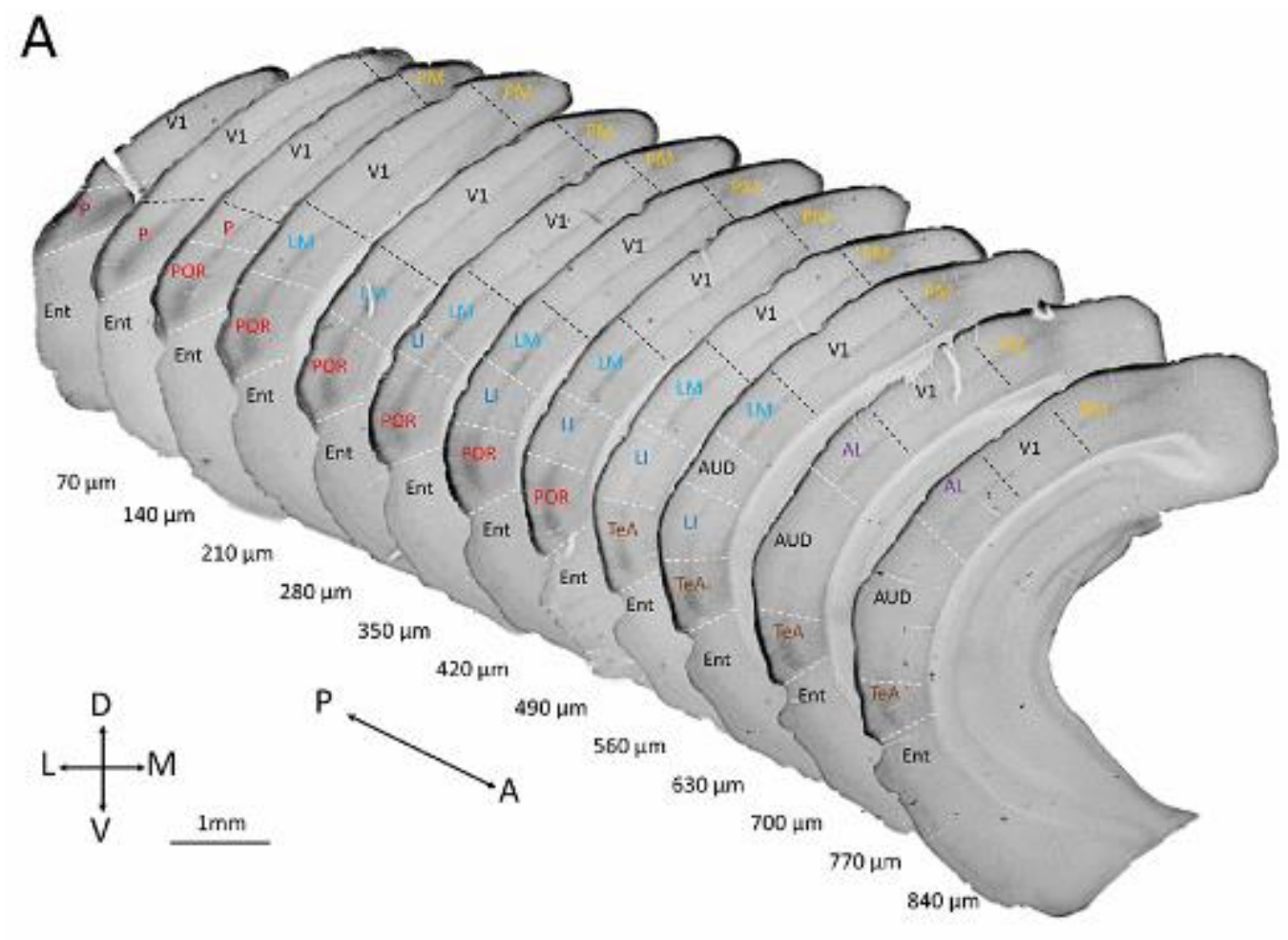

B

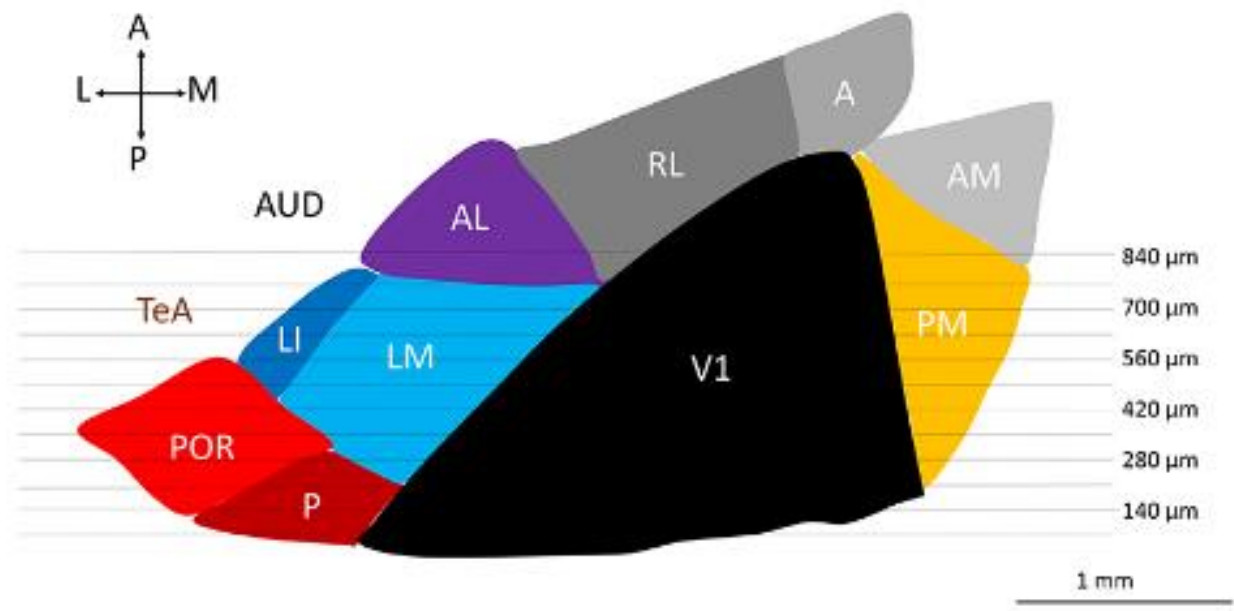




\section{Figure 30. Summary of pulvinocortical circuits}

A. Schematic of cortical cell types targeted by the pulvinar. B. Projection cell types targeted by the pulvinar (color contrast was calculated based on the percentage of responsive cells). C. Schematic of the interconnected circuits that involve the pulvinar nucleus; the pulvinar may serve as a hub to coordinate body movements with the perception of visual signals. CC, corticocortical, CS, cortico-striatal, CT, corticotectal, solid arrows indicate excitatory connections, dashed arrows indicate inhibitory connections, LES, lateral extrastriate cortex, PUL, pulvinar, SGI, stratum griseum intermediale, SGS, stratum griseum superficiale, ST, striatum. 
A

Pyramidal Interneuron Spiny stellate

B
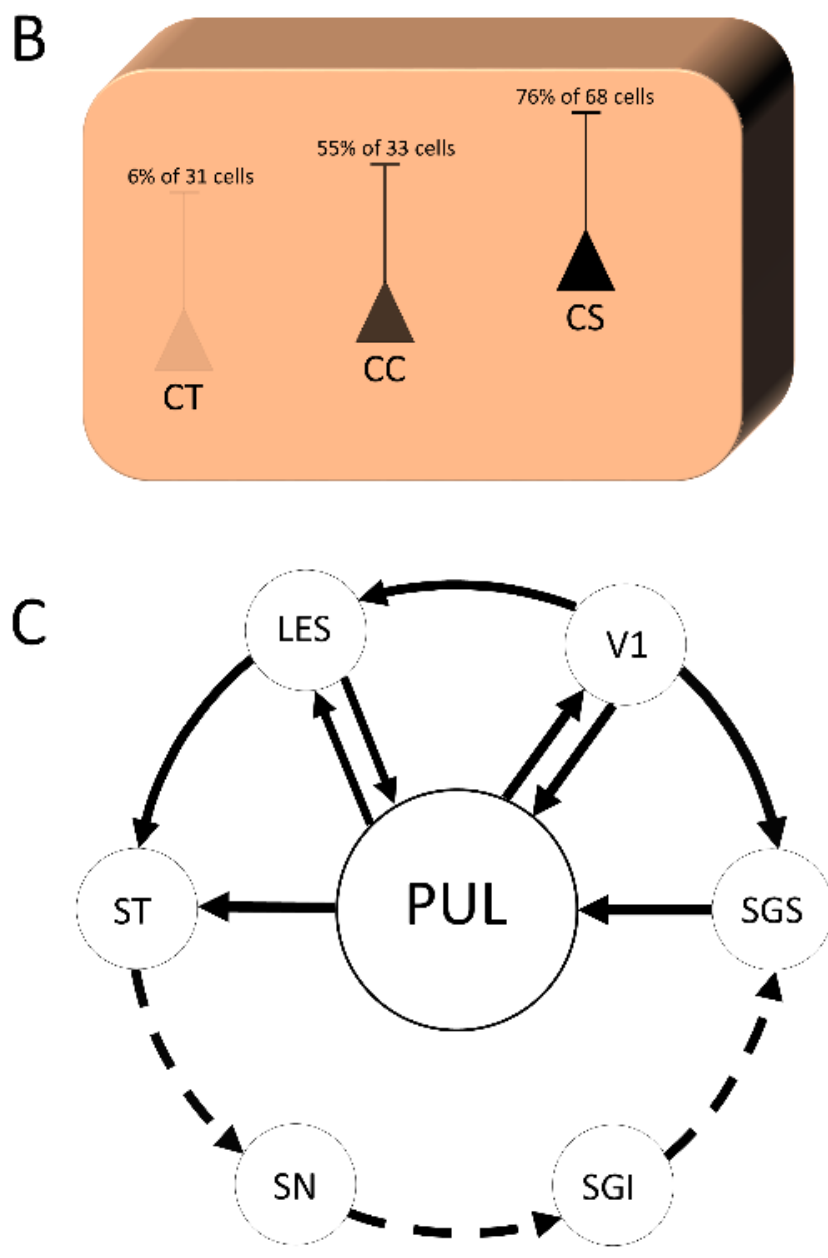


\section{CHAPTER V}

\section{CONCLUSIONS AND FUTURE DIRECTIONS}

Diamond et al. 1976 concluded that in all mammals there are two ascending visual pathways to the cortex: the retinogeniculostriate system and the tectopulvinocortical system (Diamond, 1976). However, these two systems are not completely separate from each other; in addition to the retinogeniculostriate system, both the tectogeniculate pathway and components of the tectopulvinar pathway project to the striate cortex. This dissertation focused on these two pathways from the superior colliculus to the thalamus, with the aim of understanding the synaptic organization of these parallel pathways.

In the previous chapters, I described the ultrastructure of tectogeniculate synapses in the dLGN. The convergence of retinogeniculate and tectogeniculate terminals on single cells was confirmed with electrophysiological methods. These results suggested that the SC may serve as a "backseat driver" to alert the dLGN to movement commands originating in the SC. I also characterized subdivisions of the mouse pulvinar, reviewed studies of the tectorecipient pulvinar across species, and highlighted the ongoing open questions related to tectopulvinar pathways. In the last chapter, I explored how the mouse pulvinar affects extrastriate cortical circuits. The 
results of these studies suggested that the pulvinar acts as a hub to link the extrastriate cortex with the striatum/amygdala to coordinate vision and body movement.

Many important questions regarding the tectothalamic systems remain to be answered. A few examples are: 1) How does the tectogeniculocortical pathway affect V1 circuits? 2) Are specific cortical interneuron populations targeted by pulvinocortical terminals and how do these connections affect corticostriatal output? 3) How does pulvinar input affect the receptive field properties in the LES? Below I outline the design of experiments that could address these questions.

1) How does the tectogeniculocortical pathway affect V1 circuits? It has recently been shown that certain viral vectors can cross synapses to induce expression in postsynaptic neurons. Specifically, a AAV1-hSyn-cre (Zingg et al., 2017) has been found to cross synapses. To specifically induce the expression of Chief/tdtomato in dLGN neurons that receive tectal input, the AAV1-hSyn-cre virus can be injected into the SC and a cre-dependent ChIEF/tdtomato virus can be injected into the dLGN. Then experiments similar to those described in Chapter IV could be carried out in slices of V1.

2) Are specific cortical interneuron populations targeted by pulvinocortical terminals and how do these connections affect corticostriatal output? To answer this question, an opto/chemogenetic technique could be utilized. Our initial preliminary data (Figure 31) show that cortical interneurons that contain calretinin may mainly affect the apical dendrites of pyramidal cells, whereas cortical interneurons that contain parvalbumin may primarily affect the somata of pyramidal cells. These 
specific cortical interneuron populations could be silenced using transgenic mouse lines that express "designer receptors exclusively activated by designer drugs" (DREADDs). In calretinin-cre-DREADD-GFP, parvalbumin-cre-DREADD-GFP or GAD2-cre-DREADD-GFP mice, the AAV-ChIEF viral vector could be placed in the pulvinar nucleus and CTB-546 could be placed in the striatum to induce ChIEF/tdtomato expression in pulvinocortical terminals, and identify corticostriatal cells via retrograde transport. In slices of the LES, whole cell recordings could be obtained from CTB-546-labeled corticostriatal cells and surrounding pulvinocortical terminals could be activated using pulses of blue light. Then, pulvinocortical terminals can be activated while bath applying clozapine-N-oxide (CNO) to inactivate interneurons. Comparing pulvinocortical responses before and after $\mathrm{CNO}$ application can help to determine how interneurons shape the transfer of information from the pulvinar nucleus to the striatum.

3) How does pulvinar input affect the receptive field properties in LES? To be able to answer this question, in vivo awake animal experiments need to be conducted, since previous studies have shown that anesthetics could reduce cortical cells responses and severely affect pulvinar function (Villeneuve and Casanova, 2003). Virus injections could be placed in the pulvinar nucleus to induce the expression of ARCH (a light activated hyperpolarizing proton pump, Chow et al., 2010) in pulvinocortical terminals. Extracellular recordings could then be obtained from cells in the LES using an "optrode" (a tungsten electrode with an attached fiber optic) and green light pulses (via an attached laser) could be used to silence surrounding 
pulvinocortical terminals. Comparing cortical receptive field properties before and during green light inactivation of pulvinar input could help to determine how pulvinar input contributes to LES receptive field properties. 
Figure 31. Distribution of calretinin and parvalbumin interneurons Striate

cortex sections were stained with an antibody against calretinin in A (purple) and an antibody against parvalbumin in B (green). A biocytin filled layer V pyramidal cell was placed in C (white). Calretinin expressed terminals were concentrated in layer I of striate cortex (A) where the apical dendrites of the pyramidal cell end at (C). By contrast, parvalbumin expressed terminals were completely absent at the distal dendrite of the pyramidal cell but form ring-like structure (B) around soma area of the pyramidal cell $(\mathrm{C})$. 


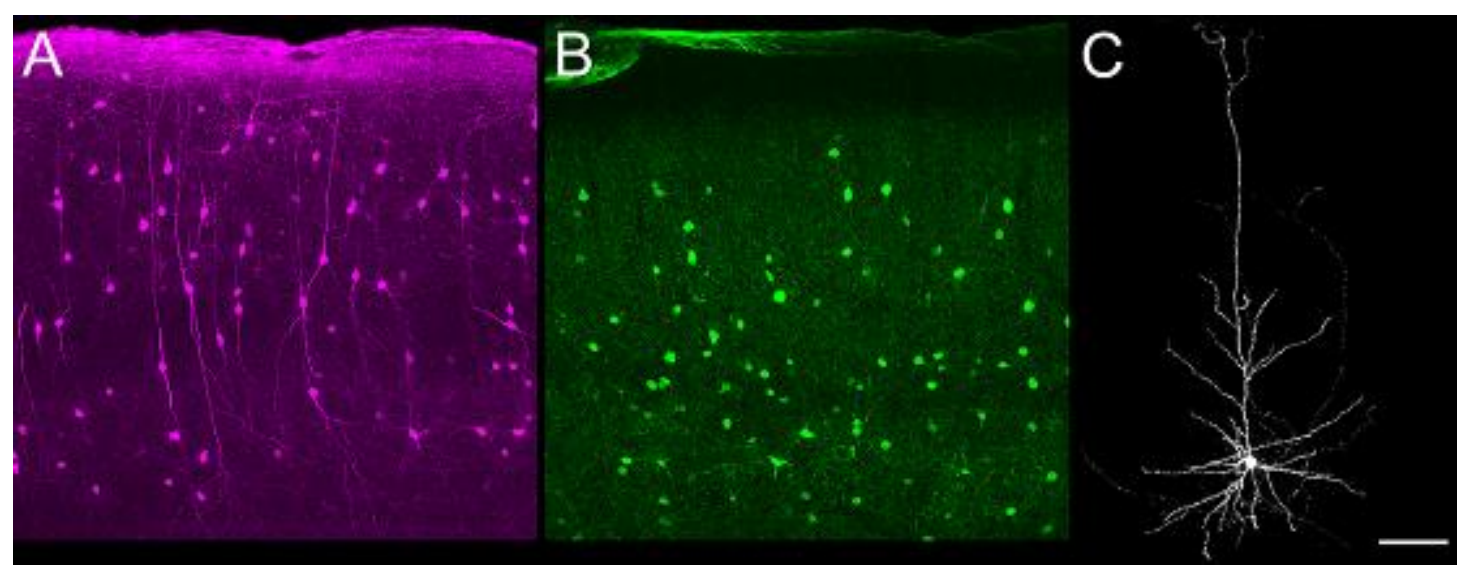




\section{REFERENCES}

Abramson BP, Chalupa LM (1985) The laminar distribution of cortical connections with the tecto- and cortico-recipient zones in the cat's lateral posterior nucleus. Neuroscience 15:81-95.

Abramson BP, Chalupa LM (1988) Multiple pathways from the superior colliculus to the extrageniculate visual thalamus of the cat. J Comp Neurol 271:397-418.

Albano JE, Norton TT, Hall WC (1979) Laminar origin of projections from the superficial layers of the superior colliculus in the tree shrew, Tupaia glis. Brain Res 173:1-11.

Allen AE, Procyk CA, Howarth M, Walmsley L, Brown TM (2016) Visual input to the mouse lateral posterior and posterior thalamic nuclei: photoreceptive origins and retinotopic order. J Physiol 594:1911-1929.

Anderson LA, Christianson GB, Linden JF (2009) Mouse auditory cortex differs from visual and somatosensory cortices in the laminar distribution of cytochrome oxidase and acetylcholinesterase. Brain Res 1252:130-142.

Arcaro MJ, Pinsk MA, Kastner S (2015) The Anatomical and Functional Organization of the Human Visual Pulvinar. J Neurosci 35:9848-9871.

Arcelli P, Frassoni C, Regondi MC, De Biasi S, Spreafico R (1997) GABAergic neurons in mammalian thalamus: a marker of thalamic complexity? Brain Res Bull 42:27-37.

Azzopardi P, Cowey A (2001) Motion discrimination in cortically blind patients. Brain 124:30-46.

Azzopardi P, Fallah M, Gross CG, Rodman HR (2003) Response latencies of neurons in visual areas MT and MST of monkeys with striate cortex lesions.

Neuropsychologia 41:1738-1756.

Baldauf ZB, Chomsung RD, Carden WB, May PJ, Bickford ME (2005a)

Ultrastructural analysis of projections to the pulvinar nucleus of the cat. I: Middle suprasylvian gyrus (areas 5 and 7). J Comp Neurol 485:87-107.

Baldauf ZB, Wang S, Chomsung RD, May PJ, Bickford ME (2005b) Ultrastructural analysis of projections to the pulvinar nucleus of the cat. II: Pretectum. J Comp Neurol 485:108-126.

Baldauf ZB, Wang X-P, Wang S, Bickford ME (2003) Pretectotectal pathway: an ultrastructural quantitative analysis in cats. J Comp Neurol 464:141-158.

Baldwin MKL, Balaram P, Kaas JH (2013) Projections of the superior colliculus to the pulvinar in prosimian galagos (Otolemur garnettii) and VGLUT2 staining of the visual pulvinar. J Comp Neurol 521:1664-1682.

Baldwin MKL, Wong P, Reed JL, Kaas JH (2011) Superior colliculus connections with visual thalamus in gray squirrels (Sciurus carolinensis): evidence for four subdivisions within the pulvinar complex. J Comp Neurol 519:1071-1094. 
Baleydier C, Mauguière F (1987) Network organization of the connectivity between parietal area 7, posterior cingulate cortex and medial pulvinar nucleus: a double fluorescent tracer study in monkey. Exp brain Res 66:385-393.

Behan M, Appell PP, Kime N (1993) Postnatal development of substance-P immunoreactivity in the rat superior colliculus. Vis Neurosci 10:1121-1127.

Benevento LA, Port JD (1995) Single neurons with both form/color differential responses and saccade-related responses in the nonretinotopic pulvinar of the behaving macaque monkey. Vis Neurosci 12:523-544.

Benshalom G, White EL (1986) Quantification of thalamocortical synapses with spiny stellate neurons in layer IV of mouse somatosensory cortex. J Comp Neurol 253:303-314.

Berman N, Cynader M (1972) Comparison of receptive-field organization of the superior colliculus in Siamese and normal cats. J Physiol 224:363-389.

Berman RA, Wurtz RH (2011) Signals conveyed in the pulvinar pathway from superior colliculus to cortical area MT. J Neurosci 31:373-384.

Berson DM, Graybiel AM (1978) Parallel thalamic zones in the LP-pulvinar complex of the cat identified by their afferent and efferent connections. Brain Res 147:139-148.

Berson DM, Graybiel AM (1983) Organization of the striate-recipient zone of the cats lateralis posterior-pulvinar complex and its relations with the geniculostriate system. Neuroscience 9:337-372.

Bickford ME (2016) Thalamic Circuit Diversity: Modulation of the Driver/Modulator Framework. Front Neural Circuits 9:86.

Bickford ME, Hall WC (1989) Collateral projections of predorsal bundle cells of the superior colliculus in the rat. J Comp Neurol 283:86-106.

Bickford ME, Hall WC (1992) The nigral projection to predorsal bundle cells in the superior colliculus of the rat. J Comp Neurol 319:11-33.

Bickford ME, Ramcharan E, Godwin DW, Erişir A, Gnadt J, Sherman SM (2000) Neurotransmitters contained in the subcortical extraretinal inputs to the monkey lateral geniculate nucleus. J Comp Neurol 424:701-717.

Bickford ME, Slusarczyk A, Dilger EK, Krahe TE, Kucuk C, Guido W (2010) Synaptic development of the mouse dorsal lateral geniculate nucleus. J Comp Neurol 518:622-635.

Bickford ME, Wei H, Eisenback M a, Chomsung RD, Slusarczyk AS, Dankowsi AB (2008) Synaptic organization of thalamocortical axon collaterals in the perigeniculate nucleus and dorsal lateral geniculate nucleus. J Comp Neurol 508:264-285.

Bickford ME, Zhou N, Krahe TE, Govindaiah G, Guido W (2015) Retinal and Tectal "Driver-Like" Inputs Converge in the Shell of the Mouse Dorsal Lateral Geniculate Nucleus. J Neurosci 35:10523-10534.

Bourassa J, Deschênes M (1995) Corticothalamic projections from the primary visual cortex in rats: a single fiber study using biocytin as an anterograde tracer. Neuroscience 66:253-263.

Bridge H, Leopold DA, Bourne JA (2016) Adaptive Pulvinar Circuitry Supports 
Visual Cognition. Trends Cogn Sci 20:146-157.

Byun H, Kwon S, Ahn H-J, Liu H, Forrest D, Demb JB, Kim I-J (2016) Molecular features distinguish ten neuronal types in the mouse superficial superior colliculus. J Comp Neurol 524:2300-2321.

Caldwell RB, Mize RR (1981) Superior colliculus neurons which project to the cat lateral posterior nucleus have varying morphologies. J Comp Neurol 203:53-66.

Callaway EM, Borrell V (2011) Developmental sculpting of dendritic morphology of layer 4 neurons in visual cortex: influence of retinal input. J Neurosci 31:74567470 .

Carden WB, Bickford ME (2002) Synaptic inputs of class III and class V interneurons in the cat pulvinar nucleus: Differential integration of RS and RL inputs. Vis Neurosci 19:51-59.

Carr JA (2015) I'll take the low road: the evolutionary underpinnings of visually triggered fear. Front Neurosci 9:414.

Casagrande VA, Harting JK, Hall WC, Diamond IT, Martin GF (1972) Superior colliculus of the tree shrew: a structural and functional subdivision into superficial and deep layers. Science 177:444-447.

Casagrande VA, Yazar F, Jones KD, Ding Y (2007) The morphology of the koniocellular axon pathway in the macaque monkey. Cereb Cortex 17:23342345.

Casanova C, Merabet L, Desautels a, Minville K (2001) Higher-order motion processing in the pulvinar. Prog Brain Res 134:71-82.

Chalupa LM, Abramson BP (1988) Receptive-field properties in the tecto- and striaterecipient zones of the cat's lateral posterior nucleus. Prog Brain Res 75:85-94.

Chalupa LM, Abramson BP (1989) Visual receptive fields in the striate-recipient zone of the lateral posterior-pulvinar complex. J Neurosci 9:347-357.

Chalupa LM, Williams RW, Hughes MJ (1983) Visual response properties in the tectorecipient zone of the cat's lateral posterior-pulvinar complex: a comparison with the superior colliculus. J Neurosci 3:2587-2596.

Chandrasekaran AR, Plas DT, Gonzalez E, Crair MC (2005) Evidence for an Instructive Role of Retinal Activity in Retinotopic Map Refinement in the Superior Colliculus of the Mouse. J Neurosci 25:6929-6938.

Chomsung RD, Petry HM, Bickford ME (2008) Ultrastructural examination of diffuse and specific tectopulvinar projections in the tree shrew. J Comp Neurol 510:2446.

Chomsung RD, Wei H, Day-Brown JD, Petry HM, Bickford ME (2010) Synaptic organization of connections between the temporal cortex and pulvinar nucleus of the tree shrew. Cereb Cortex 20:997-1011.

Chow BY, Han X, Dobry AS, Qian X, Chuong AS, Li M, Henninger M a, Belfort GM, Lin Y, Monahan PE, Boyden ES (2010) High-performance genetically targetable optical neural silencing by light-driven proton pumps. Nature 463:98102.

Cleland BG, Dubin MW, Levick WR (1971) Simultaneous recording of input and output of lateral geniculate neurones. Nat New Biol 231:191-192. 
Collins CE, Lyon DC, Kaas JH (2003) Responses of neurons in the middle temporal visual area after long-standing lesions of the primary visual cortex in adult new world monkeys. J Neurosci 23:2251-2264.

Collins CE, Xu X, Khaytin I, Kaskan PM, Casagrande V a, Kaas JH (2005) Optical imaging of visually evoked responses in the middle temporal area after deactivation of primary visual cortex in adult primates. Proc Natl Acad Sci U S A 102:5594-5599.

Conley M, Raczkowski D (1990) Sublaminar organization within layer VI of the striate cortex in Galago. J Comp Neurol 302:425-436.

Crain BJ, Hall WC (1980a) The normal organization of the lateral posterior nucleus of the golden hamster. J Comp Neurol 193:351-370.

Crain BJ, Hall WC (1980b) The organization of the lateral posterior nucleus of the golden hamster after neonatal superior colliculus lesions. J Comp Neurol 193:383-401.

Crain BJ, Hall WC (1980c) The organization of afferents to the lateral posterior nucleus in the golden hamster after different combinations of neonatal lesions. J Comp Neurol 193:403-412.

Crain BJ, Hall WC (1981) The normal organization of the lateral posterior nucleus in the golden hamster and its reorganization after neonatal superior colliculus lesions. Behav Brain Res 3:223-228.

Cruz-Martín A, El-Danaf RN, Osakada F, Sriram B, Dhande OS, Nguyen PL, Callaway EM, Ghosh A, Huberman AD (2014) A dedicated circuit links direction-selective retinal ganglion cells to the primary visual cortex. Nature 507:358-361.

Cucchiaro JB, Bickford ME, Sherman SM (1991) A GABAergic projection from the pretectum to the dorsal lateral geniculate nucleus in the cat. Neuroscience 41:213-226.

Cynader M, Berman N (1972) Receptive-field organization of monkey superior colliculus. J Neurophysiol 35:187-201.

da Costa NM, Martin KAC (2011) How thalamus connects to spiny stellate cells in the cat's visual cortex. J Neurosci 31:2925-2937.

Dankowski A, Bickford ME (2003) Inhibitory circuitry involving Y cells and Y retinal terminals in the $\mathrm{C}$ laminae of the cat dorsal lateral geniculate nucleus. J Comp Neurol 460:368-379.

Datskovskaia A, Carden WB, Bickford ME (2001) Y retinal terminals contact interneurons in the cat dorsal lateral geniculate nucleus. J Comp Neurol 430:85100 .

Day-Brown JD, Slusarczyk AS, Zhou N, Quiggins R, Petry HM, Bickford ME (2017) Synaptic organization of striate cortex projections in the tree shrew: A comparison of the claustrum and dorsal thalamus. J Comp Neurol 525:14031420 .

Day-Brown JD, Wei H, Chomsung RD, Petry HM, Bickford ME (2010) Pulvinar projections to the striatum and amygdala in the tree shrew. Front Neuroanat $4: 143$. 
De Franceschi G, Vivattanasarn T, Saleem AB, Solomon SG (2016) Vision Guides Selection of Freeze or Flight Defense Strategies in Mice. Curr Biol 26:21502154.

De Franceschi G, Vivattanasarn T, Saleem AB, Solomon SG (2016) Vision Guides Selection of Freeze or Flight Defense Strategies in Mice. Curr Biol 26:21502154.

de Gelder B, Vroomen J, Pourtois G, Weiskrantz L (1999) Non-conscious recognition of affect in the absence of striate cortex. Neuroreport 10:3759-3763.

Dean P, Redgrave P (1984) The superior colliculus and visual neglect in rat and hamster. II. Possible mechanisms. Brain Res 320:143-153.

Delwig A, Logan AM, Copenhagen DR, Ahn AH (2012) Light Evokes MelanopsinDependent Vocalization and Neural Activation Associated with Aversive Experience in Neonatal Mice Taylor WR, ed. PLoS One 7:e43787.

Dhande OS, Estevez ME, Quattrochi LE, El-Danaf RN, Nguyen PL, Berson DM, Huberman AD (2013) Genetic dissection of retinal inputs to brainstem nuclei controlling image stabilization. J Neurosci 33:17797-17813.

Dhande OS, Huberman AD (2014) Retinal ganglion cell maps in the brain: implications for visual processing. Curr Opin Neurobiol 24:133-142.

Diamond IT (1976) Organization of the visual cortex: comparative anatomical and behavioral studies. Fed Proc 35:60-67.

Diamond IT, Conley M, Fitzpatrick D, Raczkowski D (1991) Evidence for separate pathways within the tecto-geniculate projection in the tree shrew. Proc Natl Acad Sci U S A 88:1315-1319.

Diamond IT, Hall WC (1969) Evolution of neocortex. Science 164:251-262.

Dominguez-Vargas A-U, Schneider L, Wilke M, Kagan I (2017) Electrical Microstimulation of the Pulvinar Biases Saccade Choices and Reaction Times in a Time-Dependent Manner. J Neurosci 37:2234-2257.

Dong K, Qu T, Ahmed AK, Guison NG, Yamada K, Sugioka K, Yamadori T (1995) Bifurcating projections from the retinal ganglion cells to the primary visual targets (SC and LGN) in the cat. Kobe J Med Sci 41:221-234.

Donnelly JF, Thompson SM, Robertson RT (1983) Organization of projections from the superior colliculus to the thalamic lateral posterior nucleus in the rat. Brain Res 288:315-319.

Doron NN, Ledoux JE (2000) Cells in the posterior thalamus project to both amygdala and temporal cortex: a quantitative retrograde double-labeling study in the rat. J Comp Neurol 425:257-274.

Dräger UC (1975) Receptive fields of single cells and topography in mouse visual cortex. J Comp Neurol 160:269-290.

Dräger UC, Hubel DH (1975) Responses to visual stimulation and relationship between visual, auditory, and somatosensory inputs in mouse superior colliculus. J Neurophysiol 38:690-713.

Dräger UC, Hubel DH (1976) Topography of visual and somatosensory projections to mouse superior colliculus. J Neurophysiol 39:91-101.

Dumbrava D, Faubert J, Casanova C (2001) Global motion integration in the cat's 
lateral posterior-pulvinar complex. Eur J Neurosci 13:2218-2226.

Edwards MA, Schneider GE, Caviness VS (1986) Development of the crossed retinocollicular projection in the mouse. J Comp Neurol 248:410-421.

Ellis EM, Gauvain G, Sivyer B, Murphy GJ (2016) Shared and distinct retinal input to the mouse superior colliculus and dorsal lateral geniculate nucleus. $\mathrm{J}$ Neurophysiol 116:602-610.

Endo T, Tarusawa E, Notomi T, Kaneda K, Hirabayashi M, Shigemoto R, Isa T (2008) Dendritic Ih ensures high-fidelity dendritic spike responses of motion-sensitive neurons in rat superior colliculus. J Neurophysiol 99:2066-2076.

Erişir a, Van Horn SC, Sherman SM (1998) Distribution of synapses in the lateral geniculate nucleus of the cat: differences between laminae A and A1 and between relay cells and interneurons. J Comp Neurol 390:247-255.

Erisir A, Dreusicke M (2005) Quantitative morphology and postsynaptic targets of thalamocortical axons in critical period and adult ferret visual cortex. J Comp Neurol 485:11-31.

Erisir A, Van Horn SC, Sherman SM (1997) Relative numbers of cortical and brainstem inputs to the lateral geniculate nucleus. Proc Natl Acad Sci 94:15171520 .

Familtsev D, Quiggins R, Masterson SP, Dang W, Slusarczyk AS, Petry HM, Bickford ME (2016) Ultrastructure of geniculocortical synaptic connections in the tree shrew striate cortex. J Comp Neurol 524:1292-1306.

Feig S, Harting JK (1998) Corticocortical communication via the thalamus: ultrastructural studies of corticothalamic projections from area 17 to the lateral posterior nucleus of the cat and inferior pulvinar nucleus of the owl monkey. $\mathrm{J}$ Comp Neurol 395:281-295.

Finlay BL, Marder K, Cordon D (1980) Acquisition of visuomotor behavior after neonatal tectal lesions in the hamster: the role of visual experience. J Comp Physiol Psychol 94:506-518.

Fitzpatrick D (1996) The functional organization of local circuits in visual cortex: insights from the study of tree shrew striate cortex. Cereb Cortex 6:329-341.

Fitzpatrick D, Penny GR, Schmechel DE (1984) Glutamic acid decarboxylaseimmunoreactive neurons and terminals in the lateral geniculate nucleus of the cat. J Neurosci 4:1809-1829.

Florence SL, Casagrande VA (1987) Organization of individual afferent axons in layer IV of striate cortex in a primate. J Neurosci 7:3850-3868.

Franklin KBJ, Paxinos G (2008) The mouse brain in stereotaxic coordinates. Academic Press.

Fredes F, Vega-Zuniga T, Karten H, Mpodozis J (2012) Bilateral and ipsilateral ascending tectopulvinar pathways in mammals: a study in the squirrel (Spermophilus beecheyi). J Comp Neurol 520:1800-1818.

Friedlander MJ, Lin CS, Stanford LR, Sherman SM (1981) Morphology of functionally identified neurons in lateral geniculate nucleus of the cat. J Neurophysiol 46:80-129.

Gale SD, Murphy GJ (2014) Distinct representation and distribution of visual 
information by specific cell types in mouse superficial superior colliculus. $\mathrm{J}$ Neurosci 34:13458-13471.

Gale SD, Murphy GJ (2016) Active Dendritic Properties and Local Inhibitory Input Enable Selectivity for Object Motion in Mouse Superior Colliculus Neurons. J Neurosci 36:9111-9123.

Gattass R, Oswaldo-Cruz E, Sousa AP (1978) Visuotopic organization of the cebus pulvinar: a double representation the contralateral hemifield. Brain Res 152:116.

Girard P, Bullier J (1989) Visual activity in area V2 during reversible inactivation of area 17 in the macaque monkey. J Neurophysiol 62:1287-1302.

Girard P, Salin PA, Bullier J (1991) Visual activity in areas V3a and V3 during reversible inactivation of area $\mathrm{V} 1$ in the macaque monkey. J Neurophysiol 66:1493-1503.

Girard P, Salin PA, Bullier J (1992) Response selectivity of neurons in area MT of the macaque monkey during reversible inactivation of area V1. J Neurophysiol 67:1437-1446.

Goebel DJ, Pourcho RG (1992) Hydrolysis of substance P in the rabbit retina: I. Involvement of acetylcholine and acetylcholinesterase. An in vivo study. Neuropeptides 21:21-33.

Govindaiah G, Wang T, Gillette MU, Cox CL (2012) Activity-dependent regulation of retinogeniculate signaling by metabotropic glutamate receptors. J Neurosci 32:12820-12831.

Govindaiah, Cox CL (2004) Synaptic activation of metabotropic glutamate receptors regulates dendritic outputs of thalamic interneurons. Neuron 41:611-623.

Graham J, Berman N (1981) Origins of the projections of the superior colliculus to the dorsal lateral geniculate nucleus and the pulvinar in the rabbit. Neurosci Lett 26:101-106.

Graham J, Casagrande VA (1980) A light microscopic and electron microscopic study of the superficial layers of the superior colliculus of the tree shrew (Tupaia glis). J Comp Neurol 191:133-151.

Graybiel AM, Berson DM (1980) Histochemical identification and afferent connections of subdivisions in the lateralis posterior-pulvinar complex and related thalamic nuclei in the cat. Neuroscience 5:1175-1238.

Grieve KL, Acuña C, Cudeiro J (2000) The primate pulvinar nuclei: vision and action. Trends Neurosci 23:35-39.

Grimaldi P, Saleem KS, Tsao D (2016) Anatomical Connections of the Functionally Defined \&quot;Face Patches\&quot; in the Macaque Monkey. Neuron 90:13251342.

Groh A, Bokor H, Mease RA, Plattner VM, Hangya B, Stroh A, Deschenes M, Acsády L (2014) Convergence of cortical and sensory driver inputs on single thalamocortical cells. Cereb Cortex 24:3167-3179.

Groh A, de Kock CPJ, Wimmer VC, Sakmann B, Kuner T (2008) Driver or coincidence detector: modal switch of a corticothalamic giant synapse controlled by spontaneous activity and short-term depression. J Neurosci 28:9652-9663. 
Grubb MS, Thompson ID (2004) Biochemical and anatomical subdivision of the dorsal lateral geniculate nucleus in normal mice and in mice lacking the beta2 subunit of the nicotinic acetylcholine receptor. Vision Res 44:3365-3376.

Guillery RW (1969) The organization of synaptic interconnections in the laminae of the dorsal lateral geniculate nucleus of the cat. Z Zellforsch Mikrosk Anat 96:138 .

Guillery RW, Feig SL, Van Lieshout DP (2001) Connections of higher order visual relays in the thalamus: a study of corticothalamic pathways in cats. J Comp Neurol 438:66-85.

Guillery RW, Sherman SM (2002) Thalamic relay functions and their role in corticocortical communication: generalizations from the visual system. Neuron 33:163-175.

Hamos JE, Van Horn SC, Raczkowski D, Uhlrich DJ, Sherman SM, James E. Hamos, Susan C. Van Horn, Denis Raczkowski DJUSMS (1985) Synaptic connectivity of a local circuit neurone in lateral geniculate nucleus of the cat. Nature 317:618-621.

Harrell J V, Caldwell RB, Mize RR (1982) The superior colliculus neurons which project to the dorsal and ventral lateral geniculate nuclei in the cat. Exp Brain Res 46:234-242.

Harting JK, Diamond IT, Hall WC (1973a) Anterograde degeneration study of the cortical projections of the lateral geniculate and pulvinar nuclei in the tree shrew (Tupaia glis). J Comp Neurol 150:393-439.

Harting JK, Glendenning KK, Diamond IT, Hall WC (1973b) Evolution of the primate visual system: Anterograde degeneration studies of the tecto-pulvinar system. Am J Phys Anthropol 38:383-392.

Harting JK, Hall WC, Diamond IT (1972) Evolution of the pulvinar. Brain Behav Evol 6:424-452.

Harting JK, Huerta MF, Hashikawa T, van Lieshout DP (1991) Projection of the Mammalian Superior Colliculus Upon the Dorsal Lateral Geniculate Nucleus: Organization of Tectogeniculate Pathways in Nineteen Species.pdf. J Comp Neurol 304:275-306.

Harting JK, Updyke B V., Van Lieshout DP (2001) The visual-oculomotor striatum of the cat: functional relationship to the superior colliculus. Exp Brain Res 136:138-142.

Hendry SH, Yoshioka T (1994) A neurochemically distinct third channel in the macaque dorsal lateral geniculate nucleus. Science 264:575-577.

Herkenham M (1980) Laminar organization of thalamic projections to the rat neocortex. Science 207:532-535.

Hoy JL, Yavorska I, Wehr M, Niell CM (2016) Vision Drives Accurate Approach Behavior during Prey Capture in Laboratory Mice. Curr Biol 26:3046-3052.

Huberman AD, Manu M, Koch SM, Susman MW, Lutz AB, Ullian EM, Baccus SA, Barres BA (2008) Architecture and activity-mediated refinement of axonal projections from a mosaic of genetically identified retinal ganglion cells. Neuron 59:425-438. 
Huberman AD, Wei W, Elstrott J, Stafford BK, Feller MB, Barres BA (2009) Genetic identification of an On-Off direction-selective retinal ganglion cell subtype reveals a layer-specific subcortical map of posterior motion. Neuron 62:327-334.

Huppé-Gourgues F, Bickford ME, Boire D, Ptito M, Casanova C (2006) Distribution, morphology, and synaptic targets of corticothalamic terminals in the cat lateral posterior-pulvinar complex that originate from the posteromedial lateral suprasylvian cortex. J Comp Neurol 497:847-863.

Hutsler JJ, Chalupa LM (1991) Substance P immunoreactivity identifies a projection from the cat's superior colliculus to the principal tectorecipient zone of the lateral posterior nucleus. J Comp Neurol 312:379-390.

Imura K, Rockland KS (2006) Long-range interneurons within the medial pulvinar nucleus of macaque monkeys. J Comp Neurol 498:649-666.

Inayat S, Barchini J, Chen H, Feng L, Liu X, Cang J (2015) Neurons in the Most Superficial Lamina of the Mouse Superior Colliculus Are Highly Selective for Stimulus Direction. J Neurosci 35:7992-8003.

Isa $\mathrm{T}$ (2002) Intrinsic processing in the mammalian superior colliculus. Curr Opin Neurobiol 12:668-677.

Isa T, Hall WC (2009) Exploring the superior colliculus in vitro. J Neurophysiol 102:2581-2593.

Jones EG, Powell TP (1969) An electron microscopic study of the mode of termination of cortico-thalamic fibres within the sensory relay nuclei of the thalamus. Proc R Soc Lond B Biol Sci 172:173-185.

Jurgens CWD, Bell K a, McQuiston a R, Guido W (2012) Optogenetic stimulation of the corticothalamic pathway affects relay cells and GABAergic neurons differently in the mouse visual thalamus. PLoS One 7:e45717.

Kaas JH (2015) Blindsight: Post-natal Potential of a Transient Pulvinar Pathway. Curr Biol 25:R155-R157.

Kaas JH, Krubitzer LA (1992) Area 17 lesions deactivate area MT in owl monkeys. Vis Neurosci 9:399-407.

Kadoya S, Wolin LR, Massopust LC (1971) Photically evoked unit activity in the tectum opticum of the squirrel monkey. J Comp Neurol 142:495-508.

Kalil RE, Behan M (1987) Synaptic reorganization in the dorsal lateral geniculate nucleus following damage to visual cortex in newborn or adult cats. J Comp Neurol 257:216-236.

Kaneda K, Kasahara H, Matsui R, Katoh T, Mizukami H, Ozawa K, Watanabe D, Isa $\mathrm{T}$ (2011) Selective optical control of synaptic transmission in the subcortical visual pathway by activation of viral vector-expressed halorhodopsin. PLoS One 6:e18452.

Karnath HO, Himmelbach M, Rorden C (2002) The subcortical anatomy of human spatial neglect: putamen, caudate nucleus and pulvinar. Brain 125:350-360.

Karten HJ, Cox K, Mpodozis J (1997) Two distinct populations of tectal neurons have unique connections within the retinotectorotundal pathway of the pigeon (Columba livia). J Comp Neurol 387:449-465.

Karten HJ, Revzin AM (1966) The afferent connections of the nucleus rotundus in the 
pigeon. Brain Res 2:368-377.

Kaufman EF, Rosenquist a C, Raczkowski D (1984) The projections of single thalamic neurons onto multiple visual cortical areas in the cat. Brain Res 298:171-174.

Kawamura S, Diamond IT (1978) The laminar origin of descending projections from the cortex to the thalamus in Tupaia glis. Brain Res 153:333-339.

Kawamura S, Fukushima N, Hattori S, Kudo M (1980) Laminar segregation of cells of origin of ascending projections from the superficial layers of the superior colliculus in the cat. Brain Res 184:486-490.

Kay JN, De la Huerta I, Kim I-J, Zhang Y, Yamagata M, Chu MW, Meister M, Sanes JR (2011) Retinal ganglion cells with distinct directional preferences differ in molecular identity, structure, and central projections. J Neurosci 31:7753-7762.

Kelly LR, Li J, Carden WB, Bickford ME (2003) Ultrastructure and synaptic targets of tectothalamic terminals in the cat lateral posterior nucleus. J Comp Neurol 464:472-486.

Kielland A, Erisir A, Walaas SI, Heggelund P (2006) Synapsin utilization differs among functional classes of synapses on thalamocortical cells. J Neurosci 26:5786-5793.

Kim EJ, Juavinett AL, Kyubwa EM, Jacobs MW, Callaway EM (2015) Three Types of Cortical Layer 5 Neurons That Differ in Brain-wide Connectivity and Function. Neuron 88:1253-1267.

Kim I-J, Zhang Y, Meister M, Sanes JR (2010) Laminar restriction of retinal ganglion cell dendrites and axons: subtype-specific developmental patterns revealed with transgenic markers. J Neurosci 30:1452-1462.

Kim I-J, Zhang Y, Yamagata M, Meister M, Sanes JR (2008) Molecular identification of a retinal cell type that responds to upward motion. Nature 452:478-482.

Kim U, Gregory E, Hall WC (1992) Pathway from the zona incerta to the superior colliculus in the rat. J Comp Neurol 321:555-575.

Kloc M, Maffei A (2014) Target-specific properties of thalamocortical synapses onto layer 4 of mouse primary visual cortex. J Neurosci 34:15455-15465.

Kondo Y, Takada M, Honda Y, Mizuno N (1993) Bilateral projections of single retinal ganglion cells to the lateral geniculate nuclei and superior colliculi in the albino rat. Brain Res 608:204-215.

Kondo Y, Takada M, Tokuno H, Mizuno N (1994) Single retinal ganglion cells projecting in bilaterally to the lateral geniculate nuclei or superior colliculi by way of axon collaterals in the cat. J Comp Neurol 346:119-126.

Krahe TE, El-Danaf RN, Dilger EK, Henderson SC, Guido W (2011)

Morphologically distinct classes of relay cells exhibit regional preferences in the dorsal lateral geniculate nucleus of the mouse. J Neurosci 31:17437-17448.

Kravitz A V, Tye LD, Kreitzer AC (2012) Distinct roles for direct and indirect pathway striatal neurons in reinforcement. Nat Neurosci 15:816-818.

Lachica EA, Casagrande VA (1993) The morphology of collicular and retinal axons ending on small relay ( $\{\mathrm{W}\}$-like) cells of the primate lateral geniculate nucleus. Vis Neurosci 10:403-418. 
Le Q Van, Isbell LA, Matsumoto J, Le VQ, Hori E, Tran AH, Maior RS, Tomaz C, Ono T, Nishijo H (2014) Monkey pulvinar neurons fire differentially to snake postures. PLoS One 9:e114258.

Le Q Van, Isbell LA, Matsumoto J, Le VQ, Nishimaru H, Hori E, Maior RS, Tomaz C, Ono T, Nishijo H (2016) Snakes elicit earlier, and monkey faces, later, gamma oscillations in macaque pulvinar neurons. Sci Rep 6:20595.

Leopold D a (2012) Primary visual cortex: awareness and blindsight. Annu Rev Neurosci 35:91-109.

LeVay S, Gilbert CD (1976) Laminar patterns of geniculocortical projection in the cat. Brain Res 113:1-19.

Li J, Bickford ME, Guido W, Distinct WG (2003a) Distinct firing properties of higher order thalamic relay neurons. J Neurophysiol 90:291-299.

Li J, Guido W, Bickford ME (2003b) Two distinct types of corticothalamic EPSPs and their contribution to short-term synaptic plasticity. J Neurophysiol 90:34293440 .

Li J, Wang S, Bickford ME (2003c) Comparison of the ultrastructure of cortical and retinal terminals in the rat dorsal lateral geniculate and lateral posterior nuclei. $\mathrm{J}$ Comp Neurol 460:394-409.

Li K, Patel J, Purushothaman G, Marion RT, Casagrande VA (2013) Retinotopic maps in the pulvinar of bush baby ( otolemur garnettii ). J Comp Neurol 521:34323450 .

Linden R, Perry VH (1983) Massive retinotectal projection in rats. Brain Res 272:145-149.

Linke R, De Lima a D, Schwegler H, Pape HC (1999) Direct synaptic connections of axons from superior colliculus with identified thalamo-amygdaloid projection neurons in the rat: possible substrates of a subcortical visual pathway to the amygdala. J Comp Neurol 403:158-170.

López-Bendito G, Sturgess K, Erdélyi F, Szabó G, Molnár Z, Paulsen O (2004) Preferential origin and layer destination of GAD65-GFP cortical interneurons. Cereb Cortex 14:1122-1133.

Luksch H, Cox K, Karten HJ (1998) Bottlebrush dendritic endings and large dendritic fields: motion-detecting neurons in the tectofugal pathway. J Comp Neurol 396:399-414.

Luksch H, Karten HJ, Kleinfeld D, Wessel R (2001) Chattering and differential signal processing in identified motion-sensitive neurons of parallel visual pathways in the chick tectum. J Neurosci 21:6440-6446.

Lund JS, Lund RD, Hendrickson AE, Bunt AH, Fuchs AF (1975) The origin of efferent pathways from the primary visual cortex, area 17, of the macaque monkey as shown by retrograde transport of horseradish peroxidase. J Comp Neurol 164:287-303.

Luppino G, Matelli M, Carey RG, Fitzpatrick D, Diamond IT (1988) New view of the organization of the pulvinar nucleus in Tupaia as revealed by tectopulvinar and pulvinar-cortical projections. J Comp Neurol 273:67-86.

Lyon DC, Nassi JJ, Callaway EM (2010) A disynaptic relay from superior colliculus 
to dorsal stream visual cortex in macaque monkey. Neuron 65:270-279.

Ma J, Yao X-H, Fu Y, Yu Y-C (2014) Development of Layer 1 Neurons in the Mouse Neocortex. Cereb Cortex 24:2604-2618.

Maire PS, Masterson SP, Zhou N BM (2015) Parallel tectothalamic pathways in the mouse lateral posterior nucleus. Soc Neurosci Abstr 148.09.

Major DE, Luksch H, Karten HJ (2000) Bottlebrush dendritic endings and large dendritic fields: motion-detecting neurons in the mammalian tectum. J Comp Neurol 423:243-260.

Marín G, Letelier JC, Henny P, Sentis E, Farfán G, Fredes F, Pohl N, Karten H, Mpodozis J (2003) Spatial organization of the pigeon tectorotundal pathway: an interdigitating topographic arrangement. J Comp Neurol 458:361-380.

Marion R, Li K, Purushothaman G, Jiang Y, Casagrande VA (2013) Morphological and neurochemical comparisons between pulvinar and $\mathrm{V} 1$ projections to $\mathrm{V} 2$. $\mathrm{J}$ Comp Neurol 521:813-832.

Marshel JH, Garrett ME, Nauhaus I, Callaway EM (2011) Functional specialization of seven mouse visual cortical areas. Neuron 72:1040-1054.

Marshel JJHH, Kaye AAPP, Nauhaus I, Callaway EMME (2012) Anterior-Posterior Direction Opponency in the Superficial Mouse Lateral Geniculate Nucleus. Neuron 76:713-720.

Mason R (1981) Differential responsiveness of cells in the visual zones of the cat's LP-pulvinar complex to visual stimuli. Exp brain Res 43:25-33.

Mason R, Groos GA (1981) Cortico-recipient and tecto-recipient visual zones in the rat's lateral posterior (pulvinar) nucleus: an anatomical study. Neurosci Lett 25:107-112.

Masterson SP, Li J, Bickford ME (2009) Synaptic organization of the tectorecipient zone of the rat lateral posterior nucleus. J Comp Neurol 515:647-663.

Masterson SP, Li J, Bickford ME (2010) Frequency-dependent release of substance P mediates heterosynaptic potentiation of glutamatergic synaptic responses in the rat visual thalamus. J Neurophysiol 104:1758-1767.

May PJ (2006) The mammalian superior colliculus: laminar structure and connections. Prog Brain Res 151:321-378.

McFadyen J, Mermillod M, Mattingley JB, Halász V, Garrido MI (2017) A Rapid Subcortical Amygdala Route for Faces Irrespective of Spatial Frequency and Emotion. J Neurosci:3525-16.

McHaffie JG, Stanford TR, Stein BE, Coizet V, Redgrave P (2005) Subcortical loops through the basal ganglia. Trends Neurosci 28:401-407.

Miguel-Hidalgo JJ, Senba E, Takatsuji K, Tohyama M (1990) Substance P and enkephalins in the superficial layers of the rat superior colliculus: Differential plastic effects of retinal deafferentation. J Comp Neurol 299:389-404.

Miguel-Hidalgo JJ, Senba E, Takatsuji K, Tohyama M (1991) Effects of eyeenucleation on substance P-immunoreactive fibers of some retinorecipient nuclei of the rat in relation to their origin from the superior colliculus. Neuroscience 44:235-243.

Monckton JE, McCormick DA (2002) Neuromodulatory Role of Serotonin in the 
Ferret Thalamus. J Neurophysiol 87:2124-2136.

Montero VM, Scott GL (1981) Synaptic terminals in the dorsal lateral geniculate nucleus from neurons of the thalamic reticular nucleus: a light and electron microscope autoradiographic study. Neuroscience 6:2561-2577.

Montero VM, Singer W (1985) Ultrastructural identification of somata and neural processes immunoreactive to antibodies against glutamic acid decarboxylase (GAD) in the dorsal lateral geniculate nucleus of the cat. Exp brain Res 59:151165.

Mooney RD, Fish SE, Rhoades RW (1984) Anatomical and functional organization of pathway from superior colliculus to lateral posterior nucleus in hamster. $\mathrm{J}$ Neurophysiol 51:407-431.

Mooney RD, Klein BG, Rhoades RW (1985) Correlations between the structural and functional characteristics of neurons in the superficial laminae and the hamster's superior colliculus. J Neurosci 5:2989-3009.

Mooney RD, Nikoletseas MM, Ruiz SA, Rhoades RW (1988) Receptive-field properties and morphological characteristics of the superior collicular neurons that project to the lateral posterior and dorsal lateral geniculate nuclei in the hamster. J Neurophysiol 59:1333-1351.

Mort E, Cairns S, Hersch H, Finlay B (1980) The role of the superior colliculus in visually guided locomotion and visual orienting in the hamster. Physiol Psychol $8: 20-28$.

Nahmani M, Turrigiano GG (2014) Deprivation-induced strengthening of presynaptic and postsynaptic inhibitory transmission in layer 4 of visual cortex during the critical period. J Neurosci 34:2571-2582.

Nakamura H, Hioki H, Furuta T, Kaneko T (2015) Different cortical projections from three subdivisions of the rat lateral posterior thalamic nucleus: a single-neuron tracing study with viral vectors. Eur J Neurosci 41:1294-1310.

Niell CM, Stryker MP (2010) Modulation of visual responses by behavioral state in mouse visual cortex. Neuron 65:472-479.

Ogren MP, Hendrickson AE (1979a) The morphology and distribution of striate cortex terminals in the inferior and lateral subdivisions of the Macaca monkey pulvinar. J Comp Neurol 188:179-199.

Ogren MP, Hendrickson AE (1979b) The structural organization of the inferior and lateral subdivision of theMacaca monkey pulvinar. J Comp Neurol 188:147-178.

Ohara PT, Sefton AJ, Lieberman AR (1980) Mode of termination of afferents from the thalamic reticular nucleus in the dorsal lateral geniculate nucleus of the rat. Brain Res 197:503-506.

Olavarria J, Torrealba F (1978) The effect of acute lesions of the striate cortex on the retinotopic organization of the lateral peristriate cortex in the rat. Brain Res 151:386-391.

Partlow GD, Colonnier M, Szabo J (1977) Thalamic projections of the superior colliculus in the rhesus monkey, Macaca mulatta. A light and electron microscopic study. J Comp Neurol 72:285-318.

Peters A, Feldman ML (1977) The projection of the lateral geniculate nucleus to area 
17 of the rat cerebral cortex. I. General description. J Neurocytol 6:669-689.

Phongphanphanee P, Mizuno F, Lee PH, Yanagawa Y, Isa T, Hall WC (2011) A circuit model for saccadic suppression in the superior colliculus. J Neurosci 31:19491954.

Piché M, Thomas S, Casanova C (2015) Spatiotemporal profiles of receptive fields of neurons in the lateral posterior nucleus of the cat LP-pulvinar complex. $\mathrm{J}$ Neurophysiol 114:2390-2403.

Piscopo DM, El-Danaf RN, Huberman AD, Niell CM (2013) Diverse visual features encoded in mouse lateral geniculate nucleus. J Neurosci 33:4642-4656.

Purushothaman G, Marion R, Li K, Casagrande V a (2012) Gating and control of primary visual cortex by pulvinar. Nat Neurosci 15:905-912.

Rafols JA, Valverde F (1973) The structure of the dorsal lateral geniculate nucleus in the mouse. A Golgi and electron microscopic study. J Comp Neurol 150:303332.

Ramcharan EJ, Gnadt JW, Sherman SM (2005a) Higher-order thalamic relays burst more than first-order relays. Proc Natl Acad Sci U S A 102:12236-12241.

Rapaport DH, Wilson PD (1983) Retinal ganglion cell size groups projecting to the superior colliculus and the dorsal lateral geniculate nucleus in the North American opossum. J Comp Neurol 213:74-85.

Rapisardi SC, Miles TP (1984) Synaptology of retinal terminals in the dorsal lateral geniculate nucleus of the cat. J Comp Neurol 223:515-534.

Rauschecker JP (1988) Visual function of the cat's LP/LS subsystem in global motion processing. Prog Brain Res 75:95-108.

Reese BE (1984) The projection from the superior colliculus to the dorsal lateral geniculate nucleus in the rat. Brain Res 305:162-168.

Reese BE (1988) "Hidden lamination" in the dorsal lateral geniculate nucleus: the functional organization of this thalamic region in the rat. Brain Res 472:119-137.

Rhoades RW, Chalupa LM (1976) Directional selectivity in the superior colliculus of the golden hamster. Brain Res 118:334-338.

Rivlin-Etzion M, Zhou K, Wei W, Elstrott J, Nguyen PL, Barres B a, Huberman AD, Feller MB (2011) Transgenic mice reveal unexpected diversity of on-off direction-selective retinal ganglion cell subtypes and brain structures involved in motion processing. J Neurosci 31:8760-8769.

Robinson DL, McClurkin JW, Kertzman C, Petersen SE (1991) Visual responses of pulvinar and collicular neurons during eye movements of awake, trained macaques. J Neurophysiol 66:485-496.

Robinson DL, Petersen SE (1992) The pulvinar and visual salience. Trends Neurosci 15:127-132.

Robinson DL, Petersen SE, Keys W (1986) Saccade-related and visual activities in the pulvinar nuclei of the behaving rhesus monkey. Exp brain Res 62:625-634.

Robson JA, Hall WC (1977) The organization of the pulvinar in the grey squirrel (Sciurus carolinensis). II. Synaptic organization and comparisons with the dorsal lateral geniculate nucleus. J Comp Neurol 173:389-416.

Robson JA, Mason CA (1979) The synaptic organization of terminals traced from 
individual labeled retino-geniculate axons in the cat. Neuroscience 4:99-111.

Rockland KS (2002) Visual cortical organization at the single axon level: a beginning. Neurosci Res 42:155-166.

Rodman HR, Gross CG, Albright TD (1989) Afferent basis of visual response properties in area MT of the macaque. I. Effects of striate cortex removal. J Neurosci 9:2033-2050.

Rose JE, Woolsey CN (1949) Organization of the mammalian thalamus and its relationships to the cerebral cortex. Electroencephalogr Clin Neurophysiol 1:391-403-4.

Roth MM, Dahmen JC, Muir DR, Imhof F, Martini FJ, Hofer SB (2016) Thalamic nuclei convey diverse contextual information to layer 1 of visual cortex. Nat Neurosci 19:299-307.

Rovo Z, Ulbert I, Acsady L (2012) Drivers of the Primate Thalamus. J Neurosci 32:17894-17908.

Saalmann YB, Kastner S (2011) Cognitive and perceptual functions of the visual thalamus. Neuron 71:209-223.

Sauvé Y, Sawai H, Rasminsky M (2001) Topological specificity in reinnervation of the superior colliculus by regenerated retinal ganglion cell axons in adult hamsters. J Neurosci 21:951-960.

Schmid MC, Maier A (2015) To see or not to see--thalamo-cortical networks during blindsight and perceptual suppression. Prog Neurobiol 126:36-48.

Schneider GE (1969) Two visual systems. Science 163:895-902.

Scholl B, Tan AYY, Corey J, Priebe NJ (2013) Emergence of Orientation Selectivity in the Mammalian Visual Pathway. J Neurosci 33:10616-10624.

Schönitzer K, Holländer H (1984) Retinotectal terminals in the superior colliculus of the rabbit: a light and electron microscopic analysis. J Comp Neurol 223:153162.

Shepherd GM (2004) The synaptic organization of the brain. Oxford University Press.

Sherman SM (2004) Interneurons and triadic circuitry of the thalamus. Trends Neurosci 27:670-675.

Sherman SM, Guillery RW (1998) On the actions that one nerve cell can have on another: distinguishing "drivers" from "modulators". Proc Natl Acad Sci U S A 95:7121-7126.

Sherman SM, Guillery RW (2011) Distinct functions for direct and transthalamic corticocortical connections. J Neurophysiol 106:1068-1077.

Shumikhina SI (1984) [Evoked potentials in the visual and association cortex of alert cats in response to paired homogeneous stimulation of the lateral geniculate body and pulvinar]. Neirofiziologiia $=$ Neurophysiol 16:497-505.

Sillito AM, Jones HE (2002) Corticothalamic interactions in the transfer of visual information. Philos Trans R Soc Lond B Biol Sci 357:1739-1752.

Smith PH, Bartlett EL, Kowalkowski A (2007) Cortical and collicular inputs to cells in the rat paralaminar thalamic nuclei adjacent to the medial geniculate body. $\mathrm{J}$ Neurophysiol 98:681-695.

Smith D V., Clithero JA, Rorden C, Karnath H-O (2013) Decoding the anatomical 
network of spatial attention. Proc Natl Acad Sci 110:1518-1523.

Snyder M, Diamond IT (1968) The Organization and Function of the Visual Cortex in the Tree Shrew; pp. 244-263. Brain Behav Evol 1:244-263.

Snyder M, Hall WC, Diamond IT (1966) Vision in tree shrews (Tupia glis) after removal of striate cortex. Psychon Sci 6:243-244.

Soares JGM, Diogo a CM, Fiorani M, Souza a PB, Gattass R (2004) Effects of inactivation of the lateral pulvinar on response properties of second visual area cells in Cebus monkeys. Clin Exp Pharmacol Physiol 31:580-590.

Soares SC, Maior RS, Isbell LA, Tomaz C, Nishijo H (2017) Fast Detector/First Responder: Interactions between the Superior Colliculus-Pulvinar Pathway and Stimuli Relevant to Primates. Front Neurosci 11:67.

Sommer MA, Wurtz RH (2002) A pathway in primate brain for internal monitoring of movements. Science 296:1480-1482.

Sommer MA, Wurtz RH (2006) Influence of the thalamus on spatial visual processing in frontal cortex. Nature 444:374-377.

Sommer MA, Wurtz RH (2008) Brain circuits for the internal monitoring of movements. Annu Rev Neurosci 31:317-338.

Song S-H, Augustine GJ (2015) Synapsin Isoforms and Synaptic Vesicle Trafficking. Mol Cells 38:936-940.

Sparks DL, Hartwich-Young R (1989) The deep layers of the superior colliculus. Rev Oculomot Res 3:213-255.

Sprague JM (1966) Interaction of cortex and superior colliculus in mediation of visually guided behavior in the cat. Science 153:1544-1547.

Standage GP, Benevento LA (1983) The organization of connections between the pulvinar and visual area MT in the macaque monkey. Brain Res 262:288-294.

Stanford LR, Friedlander MJ, Sherman SM (1981) Morphology of physiologically identified W-cells in the $\mathrm{C}$ laminae of the cat's lateral geniculate nucleus. J Neurosci 1:578-584.

Stepniewska I, Qi HX, Kaas JH (1999) Do superior colliculus projection zones in the inferior pulvinar project to MT in primates? Eur J Neurosci 11:469-480.

Stepniewska I, Q1 HX, Kaas JH (2000) Projections of the superior colliculus to subdivisions of the inferior pulvinar in New World and Old World monkeys. Vis Neurosci 17:529-549.

Stoerig P, Cowey A (1997) Blindsight in man and monkey. Brain 120:535-559.

Straschill M, Hoffmann KP (1969) Functional aspects of localization in the cat's tectum opticum. Brain Res 13:274-283.

Takahashi T (1985) The organization of the lateral thalamus of the hooded rat. J Comp Neurol 231:281-309.

Tamamaki N, Uhlrich DJ, Sherman SM (1995) Morphology of physiologically identified retinal $\mathrm{X}$ and $\mathrm{Y}$ axons in the cat's thalamus and midbrain as revealed by intraaxonal injection of biocytin. J Comp Neurol 354:583-607.

Taniguchi H, He M, Wu P, Kim S, Paik R, Sugino K, Kvitsiani D, Kvitsani D, Fu Y, Lu J, Lin Y, Miyoshi G, Shima Y, Fishell G, Nelson SB, Huang ZJ (2011) A resource of Cre driver lines for genetic targeting of GABAergic neurons in 
cerebral cortex. Neuron 71:995-1013.

Tohmi M, Meguro R, Tsukano H, Hishida R, Shibuki K (2014) The extrageniculate visual pathway generates distinct response properties in the higher visual areas of mice. Curr Biol 24:587-597.

Trojanowski JQ, Jacobson S (1977) The morphology and laminar distribution of cortico-pulvinar neurons in the rhesus monkey. Exp brain Res 28:51-62.

Tye KM, Prakash R, Kim S-Y, Fenno LE, Grosenick L, Zarabi H, Thompson KR, Gradinaru V, Ramakrishnan C, Deisseroth K (2011) Amygdala circuitry mediating reversible and bidirectional control of anxiety. S1. Nature:1-28.

Van den Stock J, Tamietto M, Sorger B, Pichon S, Grézes J, de Gelder B (2011) Cortico-subcortical visual, somatosensory, and motor activations for perceiving dynamic whole-body emotional expressions with and without striate cortex (V1). Proc Natl Acad Sci U S A 108:16188-16193.

Van Essen DC (2005) Corticocortical and thalamocortical information flow in the primate visual system. Prog Brain Res 149:173-185.

Van Horn SC, Erişir A, Sherman SM (2000) Relative distribution of synapses in the A-laminae of the lateral geniculate nucleus of the cat. J Comp Neurol 416:509520.

Vaney DI, Peichl L, Wässle H, Illing RB (1981) Almost all ganglion cells in the rabbit retina project to the superior colliculus. Brain Res 212:447-453.

Vidnyánszky Z, Borostyánkõi Z, Görcs TJ, Hámori J (1996) Light and electron microscopic analysis of synaptic input from cortical area 17 to the lateral posterior nucleus in cats. Exp brain Res 109:63-70.

Villeneuve MY, Casanova C (2003) On the use of isoflurane versus halothane in the study of visual response properties of single cells in the primary visual cortex. $\mathrm{J}$ Neurosci Methods 129:19-31.

Waleszczyk WJ, Wang C, Benedek G, Burke W, Dreher B (2004) Motion sensitivity in cat's superior colliculus: contribution of different visual processing channels to response properties of collicular neurons. Acta Neurobiol Exp (Wars) 64:209228.

Wang L, Sarnaik R, Rangarajan K, Liu X, Cang J (2010) Visual receptive field properties of neurons in the superficial superior colliculus of the mouse. $\mathrm{J}$ Neurosci 30:16573-16584.

Wang Q, Burkhalter A (2007) Area map of mouse visual cortex. J Comp Neurol 502:339-357.

Wang Q, Burkhalter A (2013) Stream-related preferences of inputs to the superior colliculus from areas of dorsal and ventral streams of mouse visual cortex. J Neurosci 33:1696-1705.

Wang S, Bickford ME, Van Horn SC, Erisir a, Godwin DW, Sherman SM (2001) Synaptic targets of thalamic reticular nucleus terminals in the visual thalamus of the cat. J Comp Neurol 440:321-341.

Wang S, Eisenback M, Datskovskaia A, Boyce M, Bickford ME (2002) GABAergic pretectal terminals contact GABAergic interneurons in the cat dorsal lateral geniculate nucleus. Neurosci Lett 323:141-145. 
Ware CB, Casagrande VA, Diamond IT (1972) Does the acuity of the tree shrew suffer from removal of striate cortex? A commentary on the paper by ward and Masterton. Brain Behav Evol 5:18-29.

Ware CB, Diamond IT, Casagrande VA (1974) Effects of ablating the striate cortex on a successive pattern discrimination:further study of the visual system in the tree shrew (Tupaia glis). Brain Behav Evol 9:264-279.

Warner CE, Kwan WC, Bourne J a (2012) The early maturation of visual cortical area MT is dependent on input from the retinorecipient medial portion of the inferior pulvinar. J Neurosci 32:17073-17085.

Watson C (2012) Visual System. In: The Mouse Nervous System, pp 646-652. Elsevier.

Wei H, Bonjean M, Petry HM, Sejnowski TJ, Bickford ME (2011a) Thalamic burst firing propensity: a comparison of the dorsal lateral geniculate and pulvinar nuclei in the tree shrew. J Neurosci 31:17287-17299.

Wei H, Masterson SP, Petry HM, Bickford ME (2011b) Diffuse and specific tectopulvinar terminals in the tree shrew: synapses, synapsins, and synaptic potentials. PLoS One 6:e23781.

Wei P, Liu N, Zhang Z, Liu X, Tang Y, He X, Wu B, Zhou Z, Liu Y, Li J, Zhang Y, Zhou X, Xu L, Chen L, Bi G, Hu X, Xu F, Wang L (2015) Processing of visually evoked innate fear by a non-canonical thalamic pathway. Nat Commun 6:6756.

Weiskrantz L (1986) Blindsight: A Case Study and Implications. Available at: http://books.google.com/books?id=QQ4qmAEACAAJ\&dq=blindsight + (weiskra $\mathrm{ntz}+\mathrm{l}+(1986+\mathrm{a}+\mathrm{case}+$ study + and + implications $)) \& \mathrm{hl}=\& \mathrm{~cd}=1 \&$ source $=\mathrm{gbs}$ api [Accessed April 3, 2017].

Weiskrantz L, Warrington EK, Sanders MD, Marshall J (1974) Visual Capacity in the Hemianopic Field Following a Restricted Occipital Ablation. Brain 97:709-728.

White EL, Rock MP (1979) Distribution of thalamic input to different dendrites of a spiny stellate cell in mouse sensorimotor cortex. Neurosci Lett 15:115-119.

Wickersham IR, Finke S, Conzelmann K-K, Callaway EM (2007) Retrograde neuronal tracing with a deletion-mutant rabies virus. Nat Methods 4:47-49.

Wilke M, Kagan I, Andersen RA (2013) Effects of Pulvinar Inactivation on Spatial Decision-making between Equal and Asymmetric Reward Options. J Cogn Neurosci 25:1270-1283.

Wilke M, Mueller K, Leopold DA (2009) Neural activity in the visual thalamus reflects. 106:9465-9470.

Wilke M, Turchi J, Smith K, Mishkin M, Leopold DA (2010) Pulvinar inactivation disrupts selection of movement plans. J Neurosci 30:8650-8659.

Wilson JR, Friedlander MJ, Sherman SM (1984) Fine structural morphology of identified X- and Y-cells in the cat's lateral geniculate nucleus. Proc R Soc Lond B Biol Sci 221:411-436.

Wurtz RH, McAlonan K, Cavanaugh J, Berman R a (2011) Thalamic pathways for active vision. Trends Cogn Sci 15:177-184.

Xue JT, Kim CB, Moore RJ, Spear PD (1994) Influence of the superior colliculus on responses of lateral geniculate neurons in the cat. Vis Neurosci 11:1059-1076. 
Yilmaz M, Meister M (2013) Rapid innate defensive responses of mice to looming visual stimuli. Curr Biol 23:2011-2015.

Zhao X, Chen H, Liu X, Cang J (2013) Orientation-selective responses in the mouse lateral geniculate nucleus. J Neurosci 33:12751-12763.

Zhou H, Schafer RJ, Desimone R (2016) Pulvinar-Cortex Interactions in Vision and Attention. Neuron 89:209-220.

Zhou N, Maire PS, Masterson SP BM (2017) The mouse pulvinar nucleus: organization of the tectorecipient zones. Vis Neurosci.

Zhou N, Masterson SP, Damron JK, Guido W BM (2016) Optogenetic investigation of thalamocortical circuits for active vision. Soc Neurosci Abstr 529.24.

Zingg B, Chou X-L, Zhang Z-G, Mesik L, Liang F, Tao HW, Zhang LI (2017) AAVMediated Anterograde Transsynaptic Tagging: Mapping Corticocollicular InputDefined Neural Pathways for Defense Behaviors. Neuron 93:33-47. 


\section{CURRICULUM VITAE}

$\mathrm{Na}$ Zhou

Cell: 502-794-1225

Email: n0zhou04@louisville.edu

\section{EDUCATION/TRAINING}

Institute

University of Louisville

Louisville, KY, USA

University of Louisville

Louisville, KY, USA

Lanzhou University

Lanzhou, Gansu, China

\begin{tabular}{lcl}
$\begin{array}{c}\text { Degree } \\
\text { Ph.D. candidate }\end{array}$ & $\begin{array}{c}\text { Year } \\
\text { 2014-pres. }\end{array}$ & \multicolumn{1}{c}{\begin{tabular}{c}
\multicolumn{1}{c}{ Field of study } \\
Neuroscience (visual system \\
circuitry)
\end{tabular}} \\
M.S. & $2010-2014$ & $\begin{array}{l}\text { Neuroscience (visual system } \\
\text { circuitry) }\end{array}$ \\
B.S. & $2006-2010$ & Biological Sciences
\end{tabular}

\section{RESEARCH EXPERIENCE}

2011-present: Used Optogenetic techniques to study the circuitry from LPN (homolog of pulvinar) to LES (lateral extrastriate cortex) in mice and explored the output channel of this projection.

Mentor: Dr. Martha E Bickford

$>$ 2011: Observed in vivo recording experiments and surgery procedures in tree shrew (Tupaia glis).

Mentor: Dr. Heywood M Petry

$>$ 2011: Observed in vivo taste recording experiments in rat. Mentor: Dr. Robert F. Lundy

2010: Quantified induced expression of CaMK2N1 in ischemic eye tissue from rats using Real Time Polymerase Chain Reaction to see the relationship between CaM2N1 expression and ischemia.

Mentor: Dr. Nigel G. F. Cooper

2009: Water maze behavioral tests of Chrysophanol effects on learning and memory.

Mentor: Dr. Qiang Chen

\section{RESEARCH TECHNIQUE PROFICIENCY}

\section{Optogenetics}

$>$ Mouse surgery

$>$ Stereotaxic viral vector/tracer injections

$>$ Slice preparation, whole cell patch-clamp and pClamp analysisElectron microscopy

$>$ Immunohistochemistry

$>$ Confocal microscopy

$>$ Neurolucida system tracing 


\section{HONORS AND AWARDS}

> 2010, 2011: University Scholarship \& Fellowship, University of Louisville

> 2007, 2008, 2009: University Scholarship, Lanzhou University, China

$>$ 2008: My group won the third place in the Innovation and Entrepreneurship program, Study on Soil Bacteria Community Diversity of Jinchuan Nickel Mine, Lanzhou University, China

$>$ 2008: Represented my university to attend the joint field practice of biology with eight other universities around the nation in Xi' an, Shaanxi province, China

$>$ 2007,2008: Champion of woman's singles badminton tournament, Lanzhou University, China

> 2007: Excellent Volunteer Guide, Green Camel Bell-Gansu Environmental NGO, China

\section{TEACHING EXPERIENCE}

2012: Teaching assistant, Neuroanatomy (ASNB 607), School of Medicine, University of Louisville

\section{TRAINING EXPERIENCE}

2015-2016 Phillip S Maire (research assistant)

> 2014 (summer) James K Damron (medical student)

$>2013$ (summer) Wenhao Dang (graduate student)

> 2012 (summer) Jacob D Edwards (medical student)

2012 (9/17/12-2/5/13fall) Kathryn M DeVeau (rotating $1^{\text {st }}$ year graduate student)

$2012(9 / 17 / 12-2 / 19 / 13)$ Sokhadze E Guela (rotating $1^{\text {st }}$ year graduate student)

\section{VOLUNTEER EXPERIENCE}

> 4/3/2015 "NanoDays", Kentucky Science Center (Louisville Chapter of the Society for Neuroscience) helped school children to experience neuroscience.

> 5/21/2016 "Walk MS", Louisville Charity event (Louisville Chapter of the Society for Neuroscience) helped setup, and registration and handed water to walkers.

$>$ 2007, 2008, 2009 Volunteer leader of Darwin bird-watching group and public outreach to promote biologic knowledge.

2007, 2008 Volunteer for Camel Bell-Gansu Environment NGO activities to promote environmental protection.

7/13-7/25/2007 Volunteer teacher at rural countryside school in Lintao, Gansu, China. 


\section{PEER-REVIEWED PUBLICATIONS}

Zhou N, Maire PS, Masterson SP, and Bickford ME. The mouse pulvinar nucleus: organization of the tectorecipient zones. Visual Neurosci. 2017 June 27. doi: 10.1017/S0952523817000050. (Review)

Day-Brown JD, Slusarczyk AS, Zhou N, Quiggins R, Petry HM, Bickford ME. Synaptic organization of striate cortex projections in the tree shrew: A comparison of the claustrum and dorsal thalamus. J Comp Neurol. 2016 Mar 12. doi: 10.1002/cne.23998.

Bickford ME, Zhou N, Krahe TE, Govindaiah G, Guido W. Retinal and Tectal "Driver-Like" Inputs Converge in the Shell of the Mouse Dorsal Lateral Geniculate Nucleus. J Neurosci. 2015 Jul 22;35(29):10523-34. doi: 10.1523/JNEUROSCI.337514.2015 .

\section{MANUSCRIPT IN REVISION}

Zhou N, Masterson SP, Damron JK, Guido W, Bickford ME. Optogenetic investigation of thalamocortical circuits for active vision: the mouse pulvinar links the lateral extrastriate cortex, striatum, and amygdala. J Neurosci. 2017

\section{SELECTED ABSTRACTS/PRESENTATIONS}

1. Zhou N, Masterson SP, Damron JK, Guido W, Bickford ME. (2016). Optogenetic investigation of thalamocortical circuits for active vision. Society for Neuroscience Abstracts, Program \# 529.24/WW12.

2. Zhou N, Maire PS, Masterson SP, Slusarczyk A, Edwards J, Bickford ME. (2015). Thalamic circuits for active vision: parallel pathways of the mouse lateral posterior nucleus. Gordon Research Conference \& Seminar (Eye movements) Abstracts \# 41.

3. Masterson SP, Govindaiah G, Zhou N, Maire PS, Guido W, Bickford ME. (2015). Pretectal modulation of retinogeniculate signal transmission. Society for Neuroscience Abstracts, Program \# 148.06/K32.

4. Maire PS, Masterson SP, Zhou N, Bickford ME. (2015). Parallel tectothalamic pathways in the mouse lateral posterior nucleus. Society for Neuroscience Abstracts, Program \# $148.09 / \mathrm{K} 35$.

5. Damron JK, Zhou N, Bickford ME. (2015). Parallel thalamocortical pathways to the primary visual cortex: an ultrastructural analysis. Research!Louisville Abstracts.

6. Zhou N, Masterson SP, Govindaiah G, Guido W, Bickford ME. (2014). The mouse lateral posterior nucleus links the extrastriate visual cortex with the striatum and amygdala: An optogenetic investigation. Society for Neuroscience Abstracts, Program \# 817.05/EE11.

7. Zhou N, Masterson SP, Guido W, Bickford ME. (2014). Optogenetic characterization of thalamocortical synaptic targets in the mouse lateral extra striate cortex (LES). Louisville Chapter of the Society for Neuroscience Abstract \# 24. 
8. Zhou N. Optogenetic and anatomical characterization of thalamocortical synaptic targets in the mouse lateral extrastriate cortex. (2014). Kentucky Innovation and Entrepreneurship Conference Abstract \# 37.

9. Bickford ME, Zhou N, Krahe TE, Govindaiah G, Guido W. (2014). Convergence of "driver-like" inputs in the direction-selective zone of the mouse visual thalamus. Society for Neuroscience Abstracts, Program \# 817.06/EE12.

10. Masterson SP, Akers BK, Dang WH, Zhou N, Govindaiah G, Guido W, Bickford ME. (2014). Ultrastructural and optogenetic dissection of V1 corticotectal synapses in the mouse. Society for Neuroscience Abstracts, Program \#817.01/EE7.

11. Sokhadze G, Zhou N, Bickford ME, Guido W. (2014). The organization and development of cholinergic brainstem input to the mouse visual thalamus. Society for Neuroscience Abstracts, Program \#817.02/EE8.

12. Damron JK, Zhou N, Maire P, Bickford ME. (2014). Ultrastructural and optogenetic characterization of thalamocortical projections originating from the mouse lateral posterior nucleus. Research!Louisville Abstracts.

13. Zhou N, Masterson SP, Guido W, Bickford ME. (2013). Optogenetic characterization of thalamocortical synaptic targets in the mouse lateral extrastriate cortex. Society for Neuroscience Abstracts, Program \# 64.09/GG17.

14. Zhou N, Krahe TE, Jurgens CWD, Guido W, Bickford ME. (2012). Ultrastructure of mouse tectogeniculate terminals. Society for Neuroscience Abstracts, Program \#880.15/BB5. 